\title{
Symmetry-Based Pulse Sequences in Magic-Angle Spinning Solid-State NMR
}

\author{
Malcolm H. Levitt \\ Volume 9, pp 165-196 \\ in \\ Encyclopedia of Nuclear Magnetic Resonance \\ Volume 9: Advances in NMR \\ (ISBN 047149082 2) \\ Edited by \\ David M. Grant and Robin K. Harris \\ (C) John Wiley \& Sons, Ltd, Chichester, 2002
}




\section{Symmetry-Based Pulse Sequences in Magic-Angle Spinning Solid-State NMR}

\author{
Malcolm H. Levitt \\ Chemistry Department, Southampton University, Southampton, UK
}

1 Introduction

2 Homonuclear Sequences

3 Heteronuclear Sequences

4 Applications

5 Conclusions

6 References

\section{INTRODUCTION}

Much modern solid-state NMR technology is based on two physical manipulations: (i) rotation of the sample in space, usually at a fixed frequency around a fixed axis, but sometimes using more complicated trajectories; and (ii) rotation of the nuclear spin polarizations using applied rf fields.

Magic-angle spinning was first demonstrated independently by Andrew and Lowe $\mathrm{e}^{\mathbf{1 , 2}}$ in 1959. These researchers demonstrated a noticeable narrowing effect on the NMR spectra of abundant spins in solids when the sample was rotated at several $\mathrm{kHz}$ around an axis tilted by the angle $\arctan \sqrt{2}=54.74^{\circ}$ with respect to the main magnetic field (see Magic Angle Spinning, Volume 5). However, the magic-angle spinning (MAS) method was not employed much at first because it was thought to be ineffective unless the spinning frequency greatly exceeded the size of the anisotropic nuclear spin interactions - a condition difficult to satisfy except for a limited number of samples. The situation changed at the end of the 1970s, when it was found that magic-angle spinning dramatically narrowed the protondecoupled NMR peaks of isolated ${ }^{13} \mathrm{C}$ spins in organic solids. ${ }^{3}$ The ${ }^{13} \mathrm{C}$ peaks split up into narrow 'spinning sidebands', even though the spinning frequency was much smaller than the chemical shift anisotropy (CSA). MAS, in combination with Hartmann-Hahn cross-polarization and heteronuclear decoupling, became a standard method for examining the NMR spectra of rare spin species such as ${ }^{13} \mathrm{C}$ and ${ }^{15} \mathrm{~N}$ in solids. Commercial NMR companies began to offer MAS equipment as options for their spectrometers, allowing an explosion of applications.

The use of nuclear spin rotations by applied rf fields developed in parallel. The basic technique of heteronuclear decoupling was already introduced by Bloch, ${ }^{\mathbf{5}}$ and advanced rapidly in the context of solution NMR with the introduction of noise modulation ${ }^{6}$ and composite pulse decoupling ${ }^{7-9}$ (see Decoupling Methods, Volume 3). In solid-state NMR, on the other hand, heteronuclear decoupling methodology stagnated until the introduction of the two-pulse phase modulation (TPPM) by Griffin and co-workers. ${ }^{10}$ The methodology of homonuclear dipolar decoupling in solids received more attention from the start. This process started with the use of magic-angle off-resonance irradiation by Lee and Golburg ${ }^{11}$ and continued with the pulse methods of Waugh, Huber and Haeberlen, ${ }^{\mathbf{1 2 , 1 3}}$ Mansfield, ${ }^{\mathbf{1 4}}$ Rhim and Vaughan, ${ }^{15}$ Burum and co-workers ${ }^{\mathbf{1 6}}$ and many others (see Average Hamiltonian Theory, Volume 2). Advances in digital electronics made possible new approaches, such as the frequencyswitched Lee-Goldburg (FSLG) method. ${ }^{17}$ These methodological developments were accompanied by important theoretical advances such as the average Hamiltonian theory and its variants. ${ }^{13,14,18}$

For much of this period, the spatial and spin manipulations were treated separately. For example, the principles of homonuclear decoupling by applied rf fields were developed explicitly for static samples - the rotation of the sample during the rf pulse sequence, in the case of MAS, was regarded as a minor additional perturbation. Similarly, the principles of echo formation by sample rotation generally neglected the duration of applied rf pulses. This conceptual separation was made possible by an experimental separation of timescales. Routinely-available spinning frequencies were much smaller than routinely-available nutation frequencies under applied $\mathrm{rf}$ fields. This situation was not fundamentally changed even when the 'combined rotation and multiple pulse sequence' (CRAMPS) methods were introduced ${ }^{\mathbf{1 9 , 2 0}}$ (see CRAMPS, Volume 3). At the rather low spinning frequencies used initially, it was a reasonable approximation to ignore the MAS rotation during the rf pulse sequence. The enhanced narrowing effect of CRAMPS could be treated roughly by tacking the MAS rotation onto the end of a static average Hamiltonian calculation.

At the beginning of the 1980s, two developments highlighted the fact that $\mathrm{rf}$ pulse sequences and sample rotation could combine in unexpected and powerful ways. In the extraordinary sideband suppression and separation schemes of Dixon, ${ }^{21,22}$ carefully-timed sequences of strong $\pi$ pulses were applied to the rotating sample in order to manipulate the spinning sideband patterns generated by the subsequent free-induction decay. Around the same time, Waugh and coworkers applied a repetitive pulse train synchronous with the sample rotation, and showed that the averaging effect of the magic-angle rotation on the chemical shift anisotropy could be suppressed. ${ }^{23}$ This would now be called a 'CSA recoupling' experiment.

The term 'recoupling' was first used in the heteronuclear context, when it was shown that the application of a continuous rf field to one spin species may restore the effect of the heteronuclear dipolar interaction to a second spin species, by counteracting the averaging effect of the magic-angle rotation. ${ }^{\mathbf{2 4 2 5}}$ This requires selection of the $\mathrm{rf}$ amplitude so that the magic-angle rotation frequency is a small integer multiple of the nutation frequency under the rf field, a condition termed 'rotary resonance'. It was also pointed out that the same effect could be produced by applying two strong $180^{\circ}$ pulses every rotational cycle to one of the spin species. ${ }^{25}$ This idea was developed independently by Schaefer et al., leading to the highly-successful rotational echo double resonance (REDOR) method $^{26,27}$ (see REDOR \& TEDOR, 
Volume 6). As discussed below, the REDOR scheme fits into the symmetry theory of rotor-synchronized pulse sequences (in the modern notation defined below, the symmetry of the usual version of REDOR is $R 4_{2}^{1}$ ).

The next important advances were made by Tycko and co-workers. ${ }^{28,29}$ It was shown that rotor-synchronized pulse sequences, in combination with sample rotation about a nonmagic-angle axis, could be used to emulate a zero-field NMR experiment (see Zero Field NMR, Volume 8). In the context of the present article, it is important to note that Tycko used symmetry relationships to simplify the task of pulse sequence design. In the modern notation defined below, one would describe Tycko's 'zero-field in high-field' pulse schemes as having the symmetry $C 5_{1}^{1}$.

The second development made by Tycko's group also had a wide impact. The dipolar recoupling at the magic angle (DRAMA) scheme $\mathrm{30-32}^{\mathbf{3 0}}$ initiated the field of homonuclear dipolar recoupling in solids (see Homonuclear Recoupling Schemes in MAS NMR, Volume 4). DRAMA is a rather simple pulse sequence of strong $90^{\circ}$ pulses separated by intervals and is not particularly robust. A partially successful attempt was made to improve it using symmetry-based arguments. ${ }^{\mathbf{3 2}}$ A popular alternative is the radio-frequency driven recoupling (RFDR) scheme of Griffin and co-workers, which is based on strong $180^{\circ}$ pulses..$^{33,34}$ As discussed below, part of the success of RFDR may now be understood in terms of its symmetry properties (in modern notation, the symmetry of the usual version of RFDR is $R 4_{4}^{1}$ ).

A different approach to dipolar recoupling was introduced with the double-quantum homonuclear rotary resonance (2QHORROR) method. ${ }^{35}$ This was a development of the heteronuclear rotary resonance method, exploiting the additional resonance conditions associated with double-quantum transitions in homonuclear spin systems. The HORROR method showed for the first time that it is possible to achieve highly efficient 2Q excitation in a powder sample, in part by a tighter control of the orientation-dependence of the recoupled Hamiltonian. The appropriate property has come to be called " $\gamma$-encoding", and will be discussed below.

HORROR is only feasible for coupled spins with small chemical shift differences. It required a fresh theoretical approach to extend this method to more general systems while retaining the $\gamma$-encoded $2 \mathrm{Q}$ recoupling property. This effort lead to the $\mathrm{C} 7$ pulse scheme and its variants, ${ }^{\mathbf{3 6 - 4 2}}$ which are capable of efficient broadband double-quantum recoupling. In modern notation, these schemes have the symmetry $C 7_{2}^{1}$. These methods were applied to a variety of problems, including the measurement of molecular torsional angles in a membrane protein. ${ }^{\mathbf{4 3 , 4 4}}$

As well as being practically successful, the theory behind C7 was very powerful, and could be used for a variety of problems. A further extension of the symmetry theory led to a pallette of symmetries suitable for $\gamma$-encoded doublequantum recoupling, including methods which are applicable at high spinning frequencies. ${ }^{39}$ The symmetry theorems were also used to treat the problem of heteronuclear decoupling in MAS solids, generating schemes competitive with the TPPM modulation method. ${ }^{\mathbf{4 1 , 4 5}}$

In a related development, Spiess and co-workers showed that CRAMPS may be implemented at high MAS spinning frequencies by synchronizing the rf cycles with the sample rotation. ${ }^{\mathbf{4}, 47}$ In modern notation, these schemes have the symmetry $C 3_{1}^{0}$ and $C 4_{1}^{0}$.

Schemes such as $\mathrm{C} 7$ are based on repeated radio-frequency cycles, shifted in phase and synchronized with the sample rotation. Each cycle is designed to return the nuclear spins to their initial states, if other spin interactions are ignored, i.e., each cycle provides a rotation through $0^{\circ}, 360^{\circ}$, or $720^{\circ}$, etc. Although effective for double-quantum excitation, this construction scheme is too restrictive when applied to many other recoupling problems. A set of new symmetries were therefore developed, based on $180^{\circ}$ rotation elements. ${ }^{41}$ These symmetries, called $R$-symmetries, suggest many new classes of pulse sequence, and also help explain the operation of many existing schemes. As discussed below, many of the successful methods invented in the past, such as RFDR, REDOR, and TPPM conform to the $R$-symmetries. This is probably not a coincidence.

The invention of $R$-symmetries was anticipated by the through bond spectroscopy (TOBSY) method of Baldus and Meier. ${ }^{48-52}$ These authors also used symmetry arguments to design their pulse sequences, which have the modern symmetry notation $R 6_{1}^{0}$ and $R 8_{1}^{0}$.

The discovery of the $R$-symmetries is still too recent to assess their full significance. Some of the promising recent applications are discussed below.

\section{HOMONUCLEAR SEQUENCES}

\subsection{Spin Interactions}

The symmetry-based approach to the design of rotorsynchronized pulse sequences exploits the rotational properties of the nuclear spin interactions. In general, each spin interaction may be expressed as a product of three terms, expressing the transformation properties of the interaction with respect to rotations of the molecular framework ('space'), rotations of the nuclear spin polarizations ('spin') and rotations of the external magnetic field. These rotational properties may be summarized in terms of the three rotational ranks which define each nuclear spin interaction. The set of rotational ranks forms the rotational signature of a nuclear spin interaction. The rotational signatures are summarized for the most important homonuclear interactions of spin-1/2 in Table 1 .

As may be seen from Table 1, the significant nuclear spin interactions all have ranks 0,1 or 2 . For example, those interactions which are invariant to a particular type of rotation are assigned rank zero. Interactions which have the rotational symmetry of a p-orbital in atomic theory are assigned rank 1. Interactions which have the rotational symmetry of a d-orbital in atomic theory are assigned rank 2.

Consider, for example, the homonuclear dipolar interaction between nuclear spins. This has rank 2 with respect to both

Table 1 Rotational signatures of homonuclear spin interactions in diamagnetic systems of spin-1/2

\begin{tabular}{lccc}
\hline Interaction & Space rank, $l$ & Spin rank, $\lambda$ & Field rank \\
\hline Isotropic chemical shift & 0 & 1 & 1 \\
Chemical shift anisotropy & 2 & 1 & 1 \\
$J$-coupling & 0 & 0 & 0 \\
Dipole-dipole coupling & 2 & 2 & 0 \\
\hline
\end{tabular}


spatial and spin rotations, indicating, for example, that a rotation of the molecular framework by $180^{\circ}$ does not change the interaction strength, while a rotation through $90^{\circ}$ changes the sign of the interaction.

The chemical shift anisotropy interaction is more complicated, since it is a three-body interaction, involving the nuclear spins, the electronic environment and the external magnetic field. It has ranks 2, 1 and 1 with respect to molecular rotations, spin rotations, and external field rotations, respectively. This interaction changes sign if the nuclear spins are rotated by $180^{\circ}$, with the molecules and the field held fixed. The CSA interaction also changes sign if the external field is rotated through $180^{\circ}$, while the spin polarizations and the molecular environment are held fixed. The CSA interaction does not change sign, on the other hand, if the molecules are rotated through $180^{\circ}$, while the spins and the field are held fixed.

Many NMR textbooks conflate the 'spin' part and the 'field' part into one compound term, often called, confusingly, the 'spin part' of the interaction. This practice obscures the rotational properties and is best avoided.

There are also minor interactions such as the antisymmetric part of the chemical shift tensor (space, spin, field ranks of 1,1 and 1), and the $J$-coupling anisotropy (space, spin, field ranks of 2, 2 and 0 ), which are omitted here for simplicity.

The spherical ranks listed in Table 1 are defined as follows: An irreducible spherical tensor of rank $l$ has $2 l+1$ components, with a component index $m$ running from $-l$ to $+l$ in integer steps. A rotation interconverts these $2 l+1$ components according to the transformation equation:

$$
R(\Omega) T_{\lambda m} R(\Omega)^{\dagger}=\sum_{m^{\prime}=-l}^{+l} T_{\lambda m^{\prime}} D_{m^{\prime} m}^{l}(\Omega)
$$

Here $R(\Omega)$ are rotation operators and $D_{m^{\prime} m}^{l}(\Omega)$ is an element of the $(2 l+1)$-dimensional Wigner matrix. The symbol $\Omega$ indicates a triplet of Euler angles $\Omega=\{\alpha, \beta, \gamma\}$ defining a general three-dimensional rotation. Irreducible spherical tensors, Wigner matrices and Euler angles are discussed thoroughly in the literature. ${ }^{4}$ A particularly useful compilation is given in Ref. ${ }^{\mathbf{5 3}}$ The article Internal Spin Interactions \&

Rotations in Solids, Volume 4, summarizes many key results.

The Wigner elements have the following form:

$$
D_{m^{\prime} m}^{l}(\Omega)=\exp \left\{-\mathrm{i} m^{\prime} \alpha\right\} d_{m^{\prime} m}^{l}(\beta) \exp \{-\mathrm{i} m \gamma\}
$$

where $d_{m^{\prime} m}^{l}(\beta)$ is called a reduced Wigner matrix element.

Although the detailed form of the Wigner matrices and the spherical tensors depends on the rank, and the approach appears to be mathematically complicated at first sight, the transformation properties are always the same, which permits a universal approach to pulse sequence theory.

\subsection{Reference Frames}

The discussion of spin interactions in rotating solids is facilitated by defining a set of reference frames (Figure 1).

\subsubsection{The Principal Axis Frame $P^{\Lambda}$}

The principal axis frame $P^{\Lambda}$ provides the simplest form of the spin interaction $\Lambda$. For example, consider the throughspace dipole-dipole interaction (DD interaction) between two

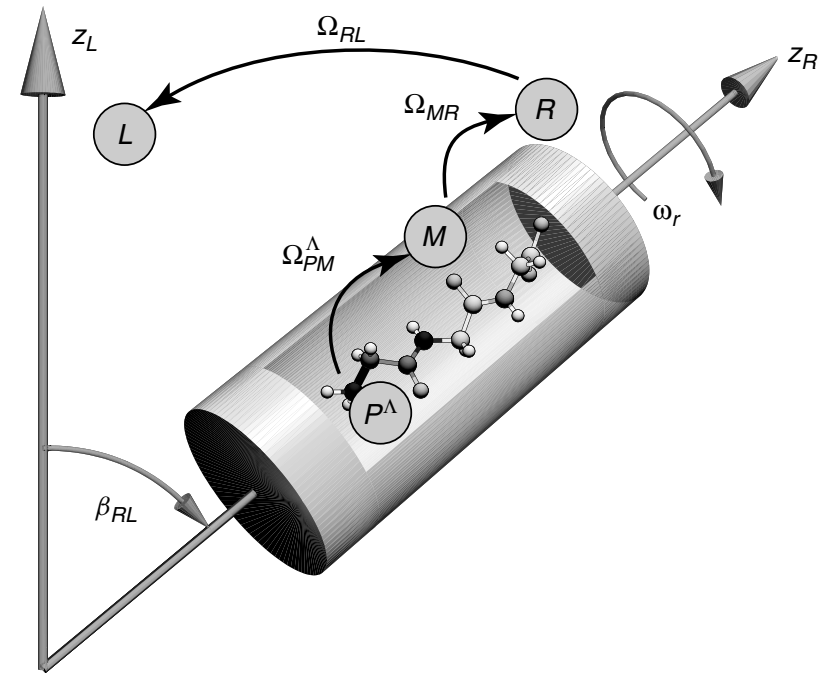

Figure 1 Definition of the reference frames in magic-angle spinning NMR experiments. (Adapted from Ref. ${ }^{\mathbf{8 2}}$ )

spins $j$ and $k$. The $z$-axis of the principal axis frame $P^{j k}$ is oriented along the vector joining the spins $j$ and $k$. In this frame, the spherical tensor components with $m= \pm 1$ and $m= \pm 2$ vanish, leaving only the $m=0$ component. For the CSA interaction of spin $j$, the $m= \pm 1$ components vanish in the principal axis frame $P^{j}$, while the $m=0$ and $m= \pm 2$ components remain finite.

In general, there are many different spin interactions, and each one has a different principal axis frame $P^{\Lambda}$.

\subsubsection{The Molecular Frame $M$}

The molecular reference frame $M$ is fixed with respect to the molecular structure. It is usual to transform all of the different spin interactions into the common reference frame $M$ before further manipulations are imposed.

A principal axis frame $P^{\Lambda}$ is related to the molecular frame $M$ by a set of three Euler angles, denoted $\Omega_{P M}^{\Lambda}=$ $\left\{\alpha_{P M}^{\Lambda}, \beta_{P M}^{\Lambda}, \gamma_{P M}^{\Lambda}\right\}$. These Euler angles define the relative orientation of the frames $P^{\Lambda}$ and $M$. In general, there are several spin interactions, each with a different set of Euler angles $\Omega_{P M}^{\Lambda}$, specifying its orientation with respect to the common molecular frame $M$.

\subsubsection{The Rotor Frame $R$}

In a spinning solid, the orientation of the molecules with respect to the rotating sample holder ('rotor') is of great importance. The rotor-fixed frame $R$ is defined such that its $z$-axis is along the rotational axis. The Euler angles $\Omega_{M R}=\left\{\alpha_{M R}, \beta_{M R}, \gamma_{M R}\right\}$ define the relative orientation of the molecular reference frame and the rotor frame.

If the sample were a single crystal, with only one molecule in the asymmetric unit of the crystal structure, then all molecules in the sample would have the same set of Euler angles $\Omega_{M R}$. In practice, the sample usually has an orientational distribution of some kind, and in the extreme case of a disordered sample such as a powder, the Euler angles $\Omega_{M R}$ are distributed randomly. In such cases, the results of experiments must be analyzed by integrating spin dynamical calculations 
over all possible values of the Euler angles $\Omega_{M R}$. This is called powder averaging.

The Euler angle $\gamma_{M R}$ has special significance in the theory of rotating solids. Molecules which have the same values of $\alpha_{M R}$ and $\beta_{M R}$ but different values of $\gamma_{M R}$ are related by a rotation around the rotor axis. This implies that the mechanical rotation of the sample causes such molecules to pass through the same cycle of laboratory frame orientations, but with a mutual time shift. This concept has been called a carousel, ${ }^{\mathbf{5 4}}$ since the riders on a playground carousel follow the same circular path, but are shifted with respect to each other in time.

In some samples, it is convenient to include one or more reference frames intermediate between $M$ and $R$. For example, in crystalline solids, it is common to define a crystalfixed reference frame $C$, and a set of Euler angles $\Omega_{M C}=$ $\left\{\alpha_{M C}, \beta_{M C}, \gamma_{M C}\right\}$ defining the orientations of the molecules with respect to the crystal axes. If there is more than one more molecule in the crystal unit cell, then several sets of Euler angles $\Omega_{M C}$ are needed. The orientation of the crystal with respect to the rotor is defined through the Euler angles $\Omega_{C R}$. In ordered samples such as fibres, on the other hand, it is useful to define a fibre director axis system $D$. The Euler angles $\Omega_{M D}$ relate the molecular axis system $M$ to the fibre director system $D$, and a further set of Euler angles $\Omega_{D R}$ relate the fibre director to the rotor axis. By factorizing the problem in this way, it is possible to define independent orientation distributions for the molecules with respect to the fibre, and for the fibres with respect to the rotor. An example of this approach is found in Refs. ${ }^{\mathbf{5 5 , 5 6}}$ If the sample is a powder, these intermediate axis systems are unnecessary, and they will be omitted in the following discussion.

\subsubsection{The Laboratory Frame $L$}

The laboratory frame is chosen so that its $z$-axis is along the external magnetic field. The relative orientation of the sample holder and the field are defined by the Euler angles $\Omega_{R L}=\left\{\alpha_{R L}, \beta_{R L}, \gamma_{R L}\right\}$.

In magic-angle spinning NMR, the sample rotates at a fixed angular frequency $\omega_{r}$ about a fixed axis, subtending the magic angle $\theta_{\text {magic }}=\arctan \sqrt{2}$ with respect to the magnetic field. In these circumstances, the relevant Euler angles are given by

$$
\begin{aligned}
& \alpha_{R L}=\alpha_{R L}^{0}-\omega_{r} t \\
& \beta_{R L}=\theta_{\text {magic }}=\arctan \sqrt{2} \\
& \gamma_{R L}=\text { arbitrary }
\end{aligned}
$$

Here $\alpha_{R L}^{0}$ defines the orientation of the rotor at time point $t=0$, which is rigorously defined as the start of signal acquisition (to be consistent with the normal definition of the Fourier transform, which involves an integral from $t=0$ to $t=\infty$ ). If the rotation of the sample is not synchronized with the pulse sequence, the angle $\alpha_{R L}^{0}$ varies randomly from transient to transient. If necessary, the rotation of the sample may be synchronized optically with the pulse sequence, so as to obtain reproducible and controllable values of $\alpha_{R L}^{0}$. This is usually unnecessary in powder samples.

The negative sign in the expression for $\alpha_{R L}$ is consistent with the usual definition of the Euler angles ${ }^{\mathbf{5 3}}$ and leads to a right-handed rotation of the sample in the case that $\omega_{r}$ is positive.

The second Euler angle $\beta_{R L}$ is the angle between the rotor axis and the field. For exact magic-angle spinning it is exactly equal to $\theta_{\text {magic }}$, but for other experiments, such as off-axis spinning, variable-axis spinning, or "zero-field in high-field" experiments, the angle $\beta_{R L}$ may take other values. In the rest of this article, exact MAS is assumed.

In double rotation or dynamical angle spinning experiments, ${ }^{57-59}$ the time-dependence of the Euler angles $\Omega_{R L}$ is more complicated (see Double Rotation, Volume 3). I ignore such possibilities here (double rotation may be handled neatly by introducing an 'outer rotor' frame as an intermediate between $R$ and $L$ ).

\subsection{Frame Transformations}

The following 'chain rule' for the Wigner matrices greatly facilitates the transformations of spin interactions between the different frames:

$$
D^{l}\left(\Omega_{A B}\right) D^{l}\left(\Omega_{B C}\right)=D^{l}\left(\Omega_{A C}\right)
$$

For example, a given spin interaction $\Lambda$ may be transformed from its principal axis frame into the laboratory frame using equation (1) and the following chain:

$$
D^{l}\left(\Omega_{P M}^{\Lambda}\right) D^{l}\left(\Omega_{M R}\right) D^{l}\left(\Omega_{R L}\right)=D^{l}\left(\Omega_{P L}^{\Lambda}\right)
$$

Note the pattern of the subscripts. This notation greatly facilitates the task of concatenating multiple frame transformations.

\subsection{Rf Euler Angles}

The mathematics of spherical tensor rotations may also be applied to the rotations of the nuclear spin polarizations by resonant rf fields. In general, a sequence of resonant rf fields induces a time-dependent rotation of the resonant spins, which may be expressed as follows:

$$
U_{\mathrm{rf}}\left(t, t^{0}\right)=\exp \left\{-\mathrm{i} \alpha_{\mathrm{rf}}(t) I_{z}\right\} \exp \left\{-\mathrm{i} \beta_{\mathrm{rf}}(t) I_{y}\right\} \exp \left\{-\mathrm{i} \gamma_{\mathrm{rf}}(t) I_{z}\right\}
$$

Here $U_{\mathrm{rf}}$ is the propagator under the applied rf fields, starting from the initial point of the pulse sequence, denoted $t^{0}$, up to an arbitrary time $t$, and $\left\{I_{x}, I_{y}, I_{z}\right\}$ are the total angular momentum operators of the resonant spin species. In general, the propagator may be determined by integrating the Schrödinger equation:

$$
\frac{\mathrm{d}}{\mathrm{d} t} U_{\mathrm{rf}}\left(t, t^{0}\right)=-\mathrm{i} \mathcal{H}_{\mathrm{rf}}(t) U_{\mathrm{rf}}\left(t, t^{0}\right)
$$

under the boundary condition $U_{\mathrm{rf}}\left(t^{0}, t^{0}\right)=\mathbf{1}$. The operator $\mathcal{H}_{\mathrm{rf}}$ is the spin Hamiltonian for the interaction with the rf field, and depends on the amplitude, phase and frequency of the applied rf field in the usual way.

In general, the rf Euler angles $\Omega_{\mathrm{rf}}=\left\{\alpha_{\mathrm{rf}}, \beta_{\mathrm{rf}}, \gamma_{\mathrm{rf}}\right\}$ do not have an obvious relationship with the applied rf pulse sequence. Nevertheless, these angles provide a convenient way of defining the symmetry properties of the spins under $\mathrm{rf}$ irradiation, and it is always possible to derive the Euler angle 
trajectory from a given pulse sequence. Furthermore, it also possible to discover the pulse sequence that provides a desired trajectory of rf Euler angles.

\subsection{Rotational Components}

The mechanical rotation of the sample induces a time-dependent trajectory of the Euler angles $\Omega_{R L}=\left\{\alpha_{R L}, \beta_{R L}, \gamma_{R L}\right\}$, while the rf pulse sequence induces a time-dependent trajectory of the Euler angles $\Omega_{\mathrm{rf}}=\left\{\alpha_{\mathrm{rf}}, \beta_{\mathrm{rf}}, \gamma_{\mathrm{rf}}\right\}$. Suppose that a certain spin interaction has space rank $l$ and spin rank $\lambda$. According to equation (1), the spatial rotation induces a mixing of the $(2 l+1)$ space components with $m=-l,-l+1 \cdots+l$. Similarly, in the interaction frame of the rf field (see Average Hamiltonian Theory, Volume 2), the spin rotations induce a mixing of the $(2 \lambda+1)$ spin components with $\mu=-\lambda,-\lambda+$ $1 \cdots+\lambda$

In the presence of sample rotation and resonant rf fields, each spin interactions may therefore be regarded as a superposition of $(2 l+1) \times(2 \lambda+1)$ components, characterized by quantum numbers $m$ and $\mu$ :

$$
\mathcal{H}^{\Lambda}=\sum_{m=-l}^{+l} \sum_{\mu=-\lambda}^{+\lambda} \mathcal{H}_{l m \lambda \mu}^{\Lambda}
$$

In the case of exact magic-angle spinning, the components with $l=2$ and $m=0$ vanish, due to the following property of the magic angle:

$$
d_{00}^{2}\left(\theta_{\text {magic }}\right)=0
$$

Explicit expressions for the components $\mathcal{H}_{\operatorname{lm} \lambda \mu}^{\Lambda}$ may be found in Refs. ${ }^{45,60}$

A summary of the spin interaction components, in the case of exact magic-angle spinning, is given in Table 2 .

\subsection{Symmetry Classes}

The space and spin trajectories may be synchronized so as to generate an average Hamiltonian containing desired combinations of quantum numbers $\{l, m, \lambda, \mu\}$ while other combinations are suppressed.

This is done by setting up periodic symmetry relationships between the mechanical and rf rotations. Consider two arbitrary time points, separated by an interval $n \tau_{r} / N$, where $n$ and $N$ are integers, and $\tau_{r}=\left|2 \pi / \omega_{r}\right|$ is the rotational period of the sample. From equation (3), the continuous rotation of the sample imposes the following relationship on the spatial Euler angles:

$$
\begin{aligned}
& \alpha_{R L}\left(t+\frac{n \tau_{r}}{N}\right)=\alpha_{R L}(t)-\frac{2 \pi n}{N} \\
& \beta_{R L}\left(t+\frac{n \tau_{r}}{N}\right)=\beta_{R L}(t)
\end{aligned}
$$

The rf pulse sequence imposes similar-looking rules on the rf Euler angles (equation (6)).

In the case of $C N_{n}^{v}$ sequences, the following Euler angle symmetry is imposed:

$$
\begin{aligned}
& \beta_{\mathrm{rf}}\left(t+\frac{n \tau_{r}}{N}\right)=\beta_{\mathrm{rf}}(t) \\
& \gamma_{\mathrm{rf}}\left(t+\frac{n \tau_{r}}{N}\right)=\gamma_{\mathrm{rf}}(t)-\frac{2 \pi v}{N}
\end{aligned}
$$

where $v$ is an integer, possibly different from $n$.

In the case of $R N_{n}^{v}$ sequences, the Euler angle symmetry is slightly different:

$$
\begin{aligned}
& \beta_{\mathrm{rf}}\left(t+\frac{n \tau_{r}}{N}\right)=\beta_{\mathrm{rf}}(t) \pm \pi \\
& \gamma_{\mathrm{rf}}\left(t+\frac{n \tau_{r}}{N}\right)=\gamma_{\mathrm{rf}}(t)-\frac{2 \pi v}{N}
\end{aligned}
$$

These symmetries apply to arbitrary time points within the pulse sequence.

The symbol $C N_{n}^{v}$ and $R N_{n}^{v}$ is called the symmetry class of the rotor-synchronized pulse sequence. The integers $N, n$ and $v$ are called the symmetry numbers of the pulse sequence. As will be seen, the symmetry class defines a set of selection rules for the average Hamiltonian, which may be used to manipulate the recoupling and decoupling properties of the pulse sequence.

\subsection{Rotor-Synchronized Pulse Sequences}

The symmetries in equations (11) and (12) only refer to two Euler angles; the behavior of the third Euler angle $\alpha_{\mathrm{rf}}$ is left completely free. This freedom implies that there are many inequivalent ways of implementing equations (11) and (12). Some simple possibilities are described below.

\subsection{1 $C N_{n}^{v}$ Sequences}

The $C N_{n}^{v}$ Euler angle symmetries [equations (10) and (11)] are generated by rotor-synchronized pulse schemes of the general form shown in Figure 2. The idea is that $n$ rotational periods of the sample are subdivided into $N$ equal intervals. Each interval contains a radio frequency pulse sequence which is a cycle, i.e., it induces a rotation of the nuclear spins through an integer multiple of $360^{\circ}$ (including zero). The radio-frequency phases of subsequent cycles are shifted with respect to each other by the angle $2 \pi \nu / N$, causing a repeated incrementation (or decrementation) of the radio-frequency phase as one proceeds through the sequence. The integers $n$ and $v$ may therefore be interpreted as space and spin winding numbers. ${ }^{39}$ The sample rotates bodily through $n$ full rotations, in the same time that it takes the radio-frequency phases to

Table 2 Components of homonuclear spin interactions in the interaction frame of an applied rf field, in the case of exact magic-angle spinning

\begin{tabular}{lcccc}
\hline Interaction & Space rank, $l$ & Space components, $m$ & Spin rank, $\lambda$ & Spin components, $\mu$ \\
\hline Isotropic chemical shift & 0 & 0 & 1 & $\{-1,0,1\}$ \\
Chemical shift anisotropy & 2 & $\{-2,-1,1,2\}$ & 1 & $\{-1,0,1\}$ \\
$J$-coupling & 0 & 0 & 0 & 0 \\
Dipole-dipole coupling & 2 & $\{-2,-1,1,2\}$ & 0 & $\{-2,-1,0,1,2\}$ \\
\hline
\end{tabular}



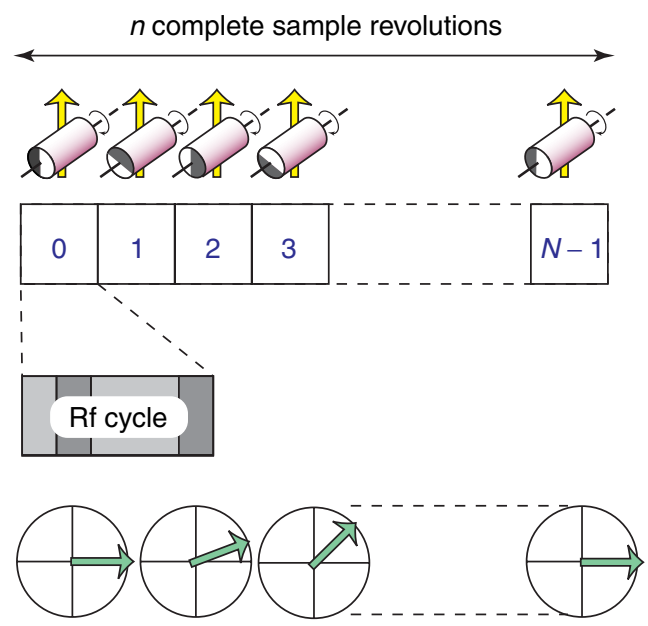

Rf phase incrementation $+2 \pi v / N$

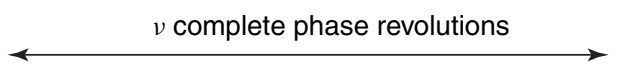

Figure 2 Construction of $C N_{n}^{v}$ sequences

(a)

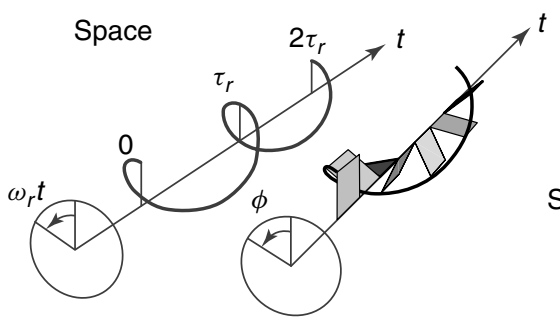

Spin

(b)

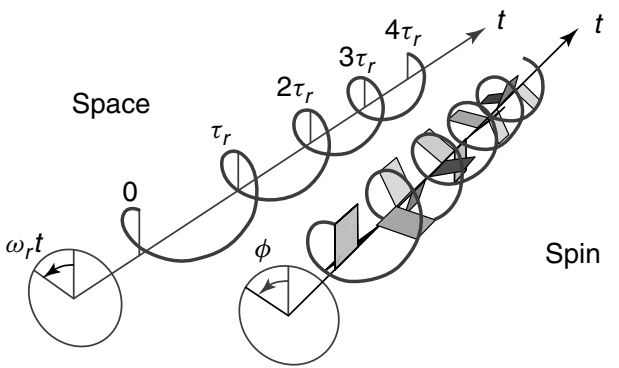

Figure 3 Visualization of the symmetry numbers for (a) $C 7_{2}^{1}$ sequences, and (b) $C 14_{4}^{5}$ sequences. The left-hand helices represent the spatial rotation of the sample as a function of time. The right-hand helices depict the modulation of the rf phase as a function of time. $n$ spatial rotations are completed in the same time as $v$ spin rotations. The rf phase is modulated in $N$ discrete steps, depicted by the planes in the right-hand diagrams. (Adapted from Ref. ${ }^{39}$ )

advance through $v$ full rotations, in $N$ equal steps. This idea is conveyed for the cases of $C 77_{2}^{1}$ and $C 14_{4}^{5}$ in Figure 3.

These symmetry properties are independent of the internal structure of the rf cycles, providing that each cycle accomplishes a final rotation through an integer multiple of $360^{\circ}$.

For a proof that sequences with this structure generate the Euler angle symmetry given in equation (11), see Ref. ${ }^{\mathbf{6 0}}$

For the sake of concreteness, consider a $C 7_{2}^{1}$ sequence based upon the cyclic element $\mathcal{C}=270_{0} 180_{180} 90_{0}$, where $\xi_{\phi}$ indicates a rectangular, resonant rf pulse with flip angle $\xi$ and phase $\phi$, and the angles are written in degrees. The full $C 7_{2}^{1}$ sequence is as follows:

$$
\begin{aligned}
& 270_{0} 180_{180} 90_{0} 270_{51.43} 180_{231.43} 90_{51.43} 270_{102.86} 180_{282.86} 90_{102.86}- \\
& 270_{154.29} 180_{334.29} 90_{154.29} 270_{205.71} 180_{25.71} 90_{205.71-} \\
& 270_{257.14} 180_{77.14} 90_{257.14} 270_{308.57} 180_{128.57} 90_{308.57}
\end{aligned}
$$

where the pulses are contiguous (no gaps in between). The rf field amplitude is chosen so that the entire sequence of 21 pulses fits exactly into two complete rotational periods of the sample. This requires that the spin nutation frequency in the rf field is exactly seven times the sample spinning frequency. The sequence in equation (13) is called POST-C7. ${ }^{37}$

\subsection{2 $R N_{n}^{v}$ Sequences}

A construction scheme for generating sequences with the Euler angle symmetries in equations (10) and (12) involves the following steps:

1. Select a sequence of $\mathrm{rf}$ pulses which rotates the nuclear spins through $180^{\circ}$ about the $x$-axis. Call this element $\mathcal{R}$.

2. Change the signs of all rf phases within the element $\mathcal{R}$. Call this phase-inverted element $\mathcal{R}^{\prime}$.

3. Select the rf amplitude so that $N$ elements $\mathcal{R}$ occupy exactly the same time interval as $n$ rotational periods of the sample. The number of elements $N$ must be even in the case of $R N_{n}^{v}$ symmetry.

4. The $R N_{n}^{v}$ sequence consists of $N / 2$ pairs of elements $\mathcal{R}_{\phi} \mathcal{R}_{-\phi}$, where $\phi$ is an overall phase shift, equal to $\phi=$ $\pi \nu / N$ radians.

A simplified diagram of this procedure is shown in Figure 4 (step 2 above is omitted for simplicity). This looks similar to the set-up for a $C N_{n}^{v}$ sequence, but with the two differences that each element provides a $180^{\circ}$ rotation, rather than a cycle, and that the rf phase is alternated between two values, rather than being incremented.

For a proof that sequences with this structure generate the Euler angle symmetry given in equation (12), see Ref. ${ }^{\mathbf{6 0}}$

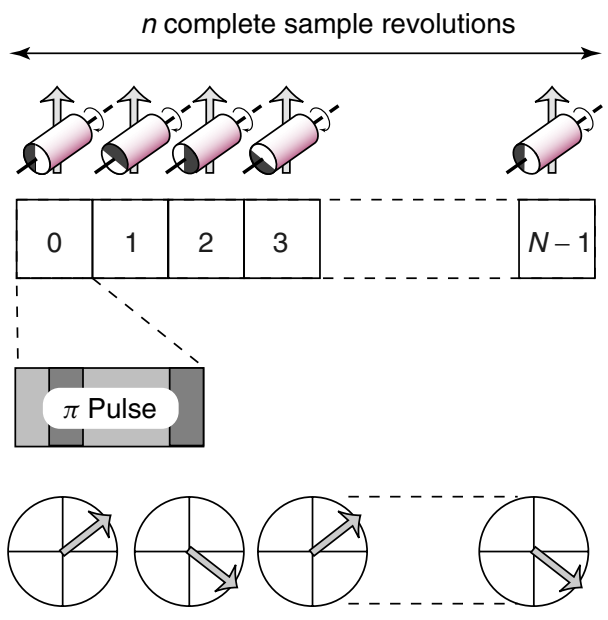

Rf phase alternation $\pm \pi \nu / N$

Figure 4 Construction of $R N_{n}^{v}$ sequences 
As a concrete example, suppose that one would like to build a $R 18_{2}^{9}$ sequence on the composite pulse $90_{0} 90_{90} 90_{0}$, which is known to have favorable homonuclear decoupling properties. ${ }^{61}$ The first step is to establish whether this element induces a $180^{\circ}$ rotation about the $x$-axis. This may be tested by rotating the three spin angular momentum operators, as follows:

$$
\begin{aligned}
& I_{x} \stackrel{90_{0}}{\longrightarrow} I_{x} \stackrel{90_{90}}{\longrightarrow}-I_{z} \stackrel{90_{0}}{\longrightarrow} I_{y} \\
& I_{y} \stackrel{90_{0}}{\longrightarrow} I_{z} \stackrel{90_{90}}{\longrightarrow} I_{x} \stackrel{90_{0}}{\longrightarrow} I_{x} \\
& I_{z} \stackrel{90_{0}}{\longrightarrow}-I_{y} \stackrel{90_{90}}{\longrightarrow}-I_{y} \stackrel{90_{0}}{\longrightarrow}-I_{z}
\end{aligned}
$$

From this it may be seen that the $90_{0} 90_{90} 90_{0}$ sequence does transform $I_{z}$ into $-I_{z}$, which is an appropriate property for a $180^{\circ}$ rotation about the $x$-axis, but that it also exchanges the operators $I_{x}$ and $I_{y}$, which is not appropriate. A little consideration leads to the conclusion that the $90_{0} 90_{90} 90_{0}$ sequence implements a rotation by $180^{\circ}$ about an axis which is intermediate between the $x$-axis and the $y$-axis, i.e., phase shifted by $45^{\circ}$. This may also be proved by rotation operator algebra. ${ }^{62}$

This defect may be remedied by phase shifting the whole sequence by $-45^{\circ}$. This leads to the element

$$
\mathcal{R}=90_{-45} 90_{45} 90_{-45}
$$

which now implements the correct rotation. Changing the sign of all phases leads to

$$
\mathcal{R}^{\prime}=90_{45} 90_{-45} 90_{45}
$$

The desired $R 18_{2}^{9}$ sequence requires additional phase shifts of $\phi= \pm \pi \times 9 / 18$ radians, or $\pm 90^{\circ}$. The appropriate elements are therefore

$$
\mathcal{R}_{\phi}=90_{45} 90_{135} 90_{45}
$$

and

$$
\mathcal{R}_{-\phi}^{\prime}=90_{-45} 90_{-135} 90_{-45}
$$

The full $R 18_{2}^{9}$ sequence has the following structure:

$$
R 18_{2}^{9}(\text { version } 1)=\left\{90_{45} 90_{135} 90_{45} 90_{-45} 90_{-135} 90_{-45}\right\}^{9}
$$

where nine repetitions of the bracketed elements occupy exactly two rotational periods of the sample. If the phases are restricted to the interval $0-360^{\circ}$, we get

$$
R 18_{2}^{9}(\text { version } 1)=\left\{90_{45} 90_{135} 90_{45} 90_{315} 90_{225} 90_{315}\right\}^{9}
$$

If the element $\mathcal{R}$ contains no internal phase shifts, or when it only contains $180^{\circ}$ phase shifts, then the conversion of $\mathcal{R}$ into $\mathcal{R}^{\prime}$ may be ignored. For example, if a single $180_{0}$ is used for the element $\mathcal{R}$, then the $R 18_{2}^{9}$ sequence is as follows:

$$
R 18_{2}^{9}(\text { version } 2)=\left\{180_{90} 180_{270}\right\}^{9}
$$

where nine repetitions of the bracketed elements occupy exactly two rotational periods of the sample.
Although the construction principles of the $R N_{n}^{v}$ sequences appear to be quite complicated, the end result is sometimes very simple.

\subsection{Effective Hamiltonian}

In many circumstances, the behavior of the nuclear spins under a pulse sequence may be described using the theory described in Average Hamiltonian Theory, Volume 2. Suppose that the pulse sequence starts at time point $t^{0}$ and lasts until time point $t^{0}+\tau$. Suppose also that the period of the pulse sequence is given by $T=n \tau_{r}$, where $n$ is the space winding number. The propagator for the pulse sequence between the time points $t^{0}$ and $t^{0}+\tau$ is written as

$$
U\left(t^{0}+\tau, t^{0}\right) \cong U_{\mathrm{rf}}\left(t^{0}+\tau, t^{0}\right) \exp \left\{-\mathrm{i} \overline{\mathcal{H}}\left(t^{0}\right) \tau\right\}
$$

where $U_{\mathrm{rf}}$ is the propagator for the rf field alone (equation (6)), $\overline{\mathcal{H}}\left(t^{0}\right)$ is the effective Hamiltonian of the internal spin interactions, for a pulse sequence starting at point $t^{0}$.

In a rotating sample, the effective Hamiltonian depends upon the starting time point $t^{0}$, since the orientation of the sample constantly changes.

The effective Hamiltonian may be approximated using the Magnus expansion:

$$
\overline{\mathcal{H}}\left(t^{0}\right) \cong \overline{\mathcal{H}}^{(1)}\left(t^{0}\right)+\overline{\mathcal{H}}^{(2)}\left(t^{0}\right)+\cdots
$$

where the first two terms are given by

$$
\begin{aligned}
& \overline{\mathcal{H}}^{(1)}\left(t^{0}\right)=T^{-1} \int_{t^{0}}^{t^{0}+T} \mathrm{~d} t \mathcal{H}_{\text {int }}(t) \\
& \overline{\mathcal{H}}^{(2)}\left(t^{0}\right)=(2 \mathrm{i} T)^{-1} \int_{t^{0}}^{t^{0}+T} \mathrm{~d} t_{2} \int_{t^{0}}^{t_{2}} \mathrm{~d} t_{1}\left[\mathcal{H}_{\text {int }}\left(t_{2}\right), \mathcal{H}_{\text {int }}\left(t_{1}\right)\right]
\end{aligned}
$$

Here $\mathcal{H}_{\text {int }}$ is the internal spin Hamiltonian, expressed in the interaction frame of the rf field. This equation employs a consistent numbering system for the Magnus expansion terms, in which the $k$ th Magnus term is proportional to a product of $k$ Hamiltonians. Much literature uses a more awkward numbering, in which the indices start at zero.

The term $\overline{\mathcal{H}}^{(1)}$ is called the average Hamiltonian. The term $\overline{\mathcal{H}}^{(2)}$ is called the second-order correction to the average Hamiltonian, and so on.

If the internal spin Hamiltonian is small compared to the period $T$ of the pulse sequence, then the average Hamiltonian expansion may be terminated after the first or second term.

Note that equation (22) represents the propagator over an arbitrary interval $\tau$, while the average Hamiltonian terms in equation (24) involve integrals over the full period of the pulse sequence. This useful approximation allows results derived for entire periodic pulse sequences to be extended, with caution, to non-integral numbers of $\mathrm{rf}$ cycles. We have generally found this approximation to work well for the symmetry-based sequences. 


\subsection{First-Order Selection Rules}

The interaction frame spin Hamiltonian is a sum of many rotational components, as in equation (8). The average Hamiltonian may therefore be written

$$
\overline{\mathcal{H}}^{(1)}\left(t^{0}\right)=\sum_{l m \lambda \mu} \overline{\mathcal{H}}_{l m \lambda \mu}^{(1)}\left(t^{0}\right)
$$

where

$$
\overline{\mathcal{H}}_{l m \lambda \mu}^{(1)}\left(t^{0}\right)=T^{-1} \int_{t^{0}}^{t^{0}+T} \mathrm{~d} t \mathcal{H}_{l m \lambda \mu}(t)
$$

Each of these components is modulated by the spatial rotation of the sample, and the rotations of the spin polarizations induced by the rf field. If the pulse sequence is a member of the $C N_{n}^{v}$ or $R N_{n}^{v}$ symmetry classes, then the synchronized modulations possess the symmetry properties summarized in equations (10)-(12).

In Refs. ${ }^{\mathbf{4 5 , 6 0}}$ it is shown that these symmetry properties lead to selection rules on the average Hamiltonian terms:

\subsubsection{Selection Rules for $C N_{n}^{v}$ Sequences}

For $C N_{n}^{v}$ sequences, the following selection rule applies:

$$
\overline{\mathcal{H}}_{l m \lambda \mu}^{(1)}=0 \quad \text { if } m n-\mu \nu \neq N Z
$$

where $Z$ is any integer, including zero.

\subsubsection{Selection Rules for $R N_{n}^{v}$ Sequences}

For $R N_{n}^{v}$ sequences, the following selection rule applies:

$$
\overline{\mathcal{H}}_{l m \lambda \mu}^{(1)}=0 \quad \text { if } m n-\mu \nu \neq \frac{N}{2} Z_{\lambda}
$$

Here $Z_{\lambda}$ is any integer with the same parity as the spin rank $\lambda$. If $\lambda$ is even, then $Z_{\lambda}$ takes the values $0, \pm 2, \pm 4, \ldots$. If $\lambda$ is odd, then $Z_{\lambda}$ takes the values $\pm 1, \pm 3, \pm 5, \ldots$.

Equations (27) and (28) are the central results of symmetrybased pulse sequence theory. These two simple equations have wide consequences.

As a first example, consider a pulse sequence with symmetry $C 7 \frac{1}{2}$. Let us examine the fate of double-quantum dipole-dipole terms with quantum numbers $\{l, m, \lambda, \mu\}=$ $\{2,1,2,2\}$. Equation (27) states that the average Hamiltonian of this term vanishes if the quantity $m n-\mu v$ is not equal to an integer multiple of $N$. Substituting in the values $m=1, n=2, \mu=2$ and $v=1$ we obtain $m n-\mu v=$ $(1 \times 2)-(2 \times 1)=0$. This is an integer multiple of $N=7$, so the term $\{l, m, \lambda, \mu\}=\{2,1,2,2\}$ is symmetry allowed. One cannot be sure whether this term actually exists without further calculation, but at least symmetry does not exclude it. Consider now the chemical shift anisotropy term with quantum numbers $\{l, m, \lambda, \mu\}=\{2,-2,1,1\}$. The expression $m n-\mu \nu$ evaluates to the value $m n-\mu \nu=((-2) \times 2)-(1 \times 1)=-5$. This is not an integer multiple of 7 , so this term is symmetry forbidden in the first-order average Hamiltonian.

Now consider the fate of the same two terms under the symmetry $R 18_{1}^{7}$, using the rule in equation (28). The homonuclear dipole-dipole terms have a spin rank $\lambda=2$, which is even. Equation (28) implies that a dipole-dipole term is symmetry-forbidden unless $m n-\mu \nu$ is equal to an even multiple of 9 . For the term $\{l, m, \lambda, \mu\}=\{2,1,2,2\}$, the expression $m n-\mu \nu$ evaluates to $(1 \times 1)-(2 \times 7)=-13$. This is not an even multiple of 9 , so the term $\{2,1,2,2\}$ is forbidden under the symmetry $R 18_{1}^{7}$. For the chemical shift anisotropy term $\{l, m, \lambda, \mu\}=\{2,-2,1,1\}$, on the other hand, the expression $m n-\mu \nu$ evaluates to the value $m n-\mu \nu=$ $((-2) \times 1)-(1 \times 7)=-9$. This is an odd multiple of 9 , and since the spin rank $\lambda$ is also odd, this term is symmetryallowed.

\subsection{Space-Spin Selection Diagrams}

The consequences of equations (27) and (28) are conveniently evaluated using space-spin selection diagrams (SSS diagrams). Two examples are given in Figure 5 and 6.

The branching lines in these diagrams show the possible values of $m n-\mu \nu$ for the particular class of spin interaction, split up into the 'space' part $m n$ and the 'spin' part $\mu \nu$. The number of branches depends on the type of spin interaction, according to Table 2, while the spacing between the branches depends on the symmetry numbers $n$ and $v$. The righthand side of each diagram shows a barrier containing holes spaced by the symmetry number $N$. Those pathways that are blocked by the barrier represent values of $m n-\mu v$ which $d o$ satisfy the inequalities in equations (27) and (28), and represent components that are symmetry-forbidden. Those pathways that pass through the holes in the barrier represent values of $m n-\mu v$ which do not satisfy the inequalities in equations (27) and (28), indicating components that are symmetry-allowed. The SSS diagrams allow the consequences of the inequalities in equations (27) and (28) to be visualized clearly.

The positions of the holes depend on the symmetry class. For the $C N_{n}^{v}$ class of symmetries, the holes in the barrier occur at levels $0, \pm N, \pm 2 N, \ldots$. For the $R N_{n}^{v}$ class of symmetries, the positions of the holes depends on whether the spin rank $\lambda$ is even or odd. For even ranks, the holes occur at levels $0, \pm N$, $\pm 2 N, \ldots$, as in the case of $C N_{n}^{v}$ symmetries. For odd ranks, on the other hand, the holes occur at levels $\pm N / 2, \pm 3 N / 2, \ldots$, where $N / 2$ is an integer, since $N$ is even.

\subsubsection{SSS Diagrams for $\mathrm{C7}_{2}^{1}$ Symmetry}

The behavior of the chemical shift anisotropy (CSA) terms under $C 7_{2}^{1}$ symmetry is shown in Figure 5(a). There are four branches in the space part, corresponding to the $m=$ $\{-2,-1,1,2\}$ components indicated in Table 2 . For clarity, only the branches with $m \leq 0$ are shown: the branches with negative $m$ are mirror images. The $m=0$ component vanishes in the case of exact magic-angle spinning. The 'space' branches are spaced vertically by two units, according to the winding number $n=2$. Each of these branches splits into three spin components, with indices $\mu=\{-1,0,1\}$, and spaced vertically by one unit, according to the winding number $v=1$. Since the value of $N$ is 7, the barrier on the right contains holes at levels $0, \pm 7, \pm 14, \ldots$. There are no CSA pathways which pass through these holes, indicating that the average Hamiltonian contains no CSA terms. The SSS diagram shows immediately that the pulse sequence is compensated for the effects of CSA, to first order. 
(a)
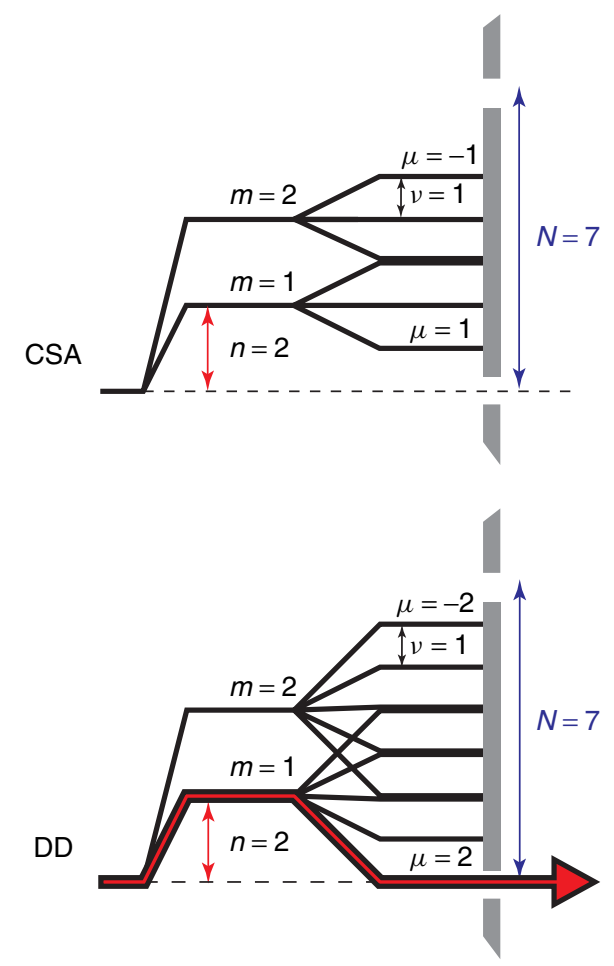

(b)

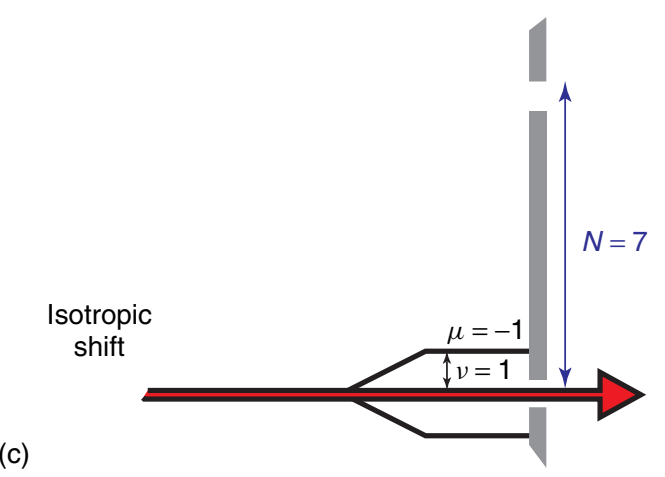

Figure 5 Space-spin selection diagram for $C 7_{2}^{1}$ symmetry, omitting components with $m<0$. (a) Behavior of the CSA terms. All terms are symmetry-forbidden. (b) Behavior of the homonuclear DD coupling terms. The component $\{l, m, \lambda, \mu\}=\{2,1,2,2\}$ is symmetry-allowed. (c) Behavior of the isotropic chemical shift terms. The component $\{l, m, \lambda, \mu\}=\{0,0,1,0\}$ is symmetry-allowed

The behavior of the dipole-dipole coupling (DD) terms under $C 7_{2}^{1}$ symmetry is shown in Figure 5(b). Here too there are four branches in the space part, corresponding to the $m=\{-2,-1,1,2\}$ components in Table 2 . Only the branches with non-negative $m$ are shown, for the sake of clarity. Each of the space branches splits into five spin branches, with indices $\mu=\{-2,-1,0,1,2\}$. As may be seen, the branch with $\{l, m, \lambda, \mu\}=\{2,1,2,2\}$ passes through a hole in the barrier, indicating that this component is symmetry-allowed. The mirror-image branch with $\{l, m, \lambda, \mu\}=\{2,-1,2,-2\}$ is also symmetry-allowed (not shown).

Since all the CSA terms are symmetry-forbidden, and the only symmetry-allowed DD terms have quantum numbers $\mu= \pm 2$, sequences with the symmetry $C 7_{2}^{1}$ behave as $C S A$ compensated double-quantum recoupling sequences.

Now consider the SSS diagram for isotropic chemical shift

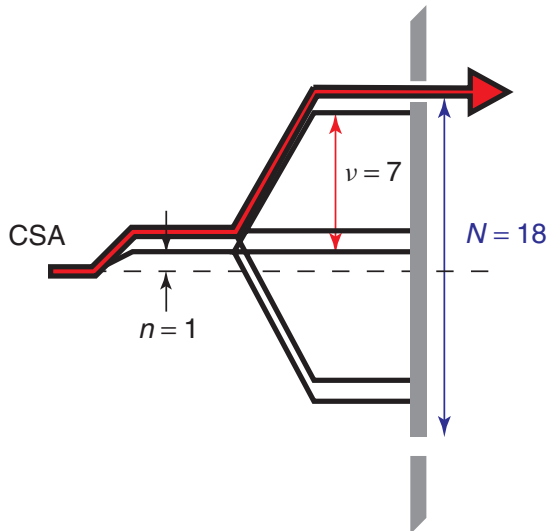

(a)

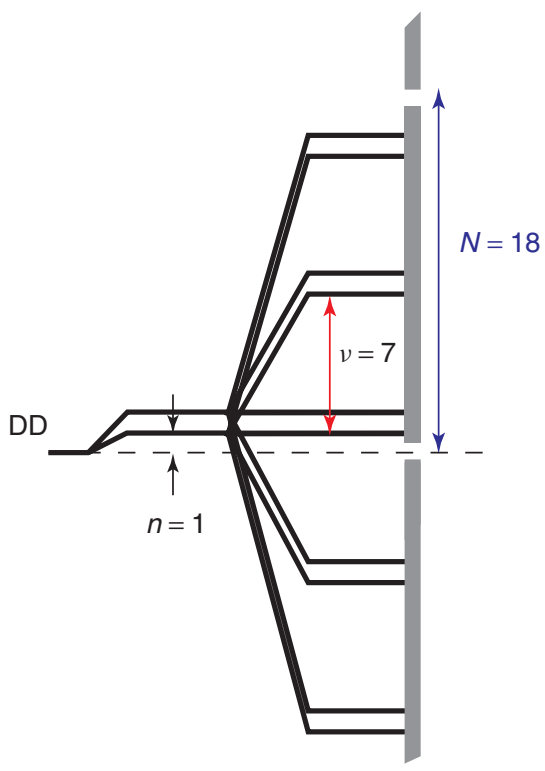

(b)

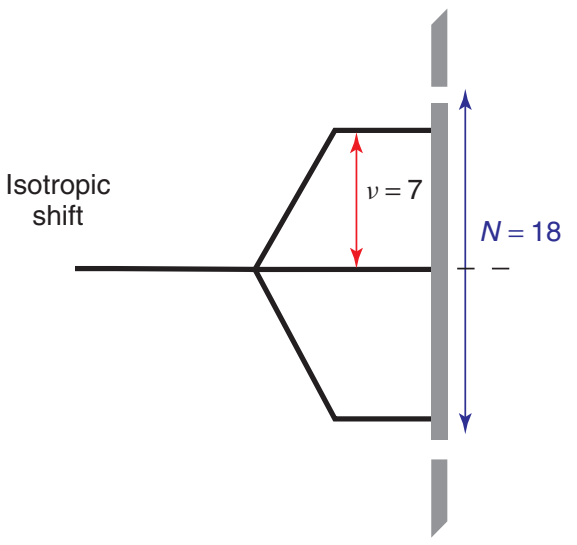

Figure 6 Space-spin selection diagram for $R 18_{1}^{7}$ symmetry, omitting components with $m<0$. (a) Behavior of the CSA terms. The component $\{l, m, \lambda, \mu\}=\{2,2,1,-1\}$ is symmetry-allowed. (b) Behavior of the homonuclear DD coupling terms. All components are symmetryforbidden. (c) Behavior of the isotropic chemical shift terms. All components are symmetry-forbidden. Note that the positions of the holes change, depending on whether the spin rank $\lambda$ is odd or even

terms, shown in Figure 5(c). Since the space and spin ranks are $l=0$ and $\lambda=1$, there is one space component, with $m=0$, 
and three spin components, with $\mu=\{-1,0,1\}$. The SSS diagram shows that the isotropic shift component $\{l, m, \lambda, \mu\}=$ $\{0,0,1,0\}$ is symmetry-allowed under $C 7_{2}^{1}$ symmetry. This implies that $C 7_{2}^{1}$ sequences are vulnerable to isotropic chemical shifts, unless additional precautions are taken. We return to this issue later.

\subsubsection{SSS Diagrams for R18 $8_{1}^{7}$ Symmetry}

The behavior of the CSA terms under $R 18_{1}^{7}$ symmetry is shown in Figure 6(a). The left-hand side shows the two space branches with $m=1$ and 2 . The $m=0$ component vanishes in the case of exact magic-angle spinning. The space branches are spaced vertically by 1 unit, according to the winding number $n=1$. Each branch splits into three spin components, with indices $\mu=\{-1,0,1\}$, and spaced vertically by 7 units, according to the winding number $v=7$. Since the spin rank $\lambda$ of the CSA terms is equal to 1 , which is an odd number, the barrier on the right contains holes at odd multiples of $N / 2$, which is equal to 9 in this case. The holes in the barrier are therefore at levels $\pm 9, \pm 27, \ldots$. As may be seen, the CSA term $\{l, m, \lambda, \mu\}=\{2,2,1,-1\}$ passes through a hole, which indicates that $R 18_{1}^{7}$ symmetry is a CSA recoupling sequence.

The behavior of the DD terms under $R 18_{1}^{7}$ symmetry is shown in Figure 6(b). The space branches with $m=1$ and 2 split into five spin components, with indices $\mu=$ $\{-2,-1,0,1,2\}$. In the case of DD couplings, the spin rank $\lambda$ is equal to 2 , which is an even number. As a result, the barrier on the right contains holes at even multiples of 9, i.e., $0, \pm 18, \pm 36, \ldots$. As may be seen, the barrier blocks all of the DD terms. This indicates that sequences with $R 18_{1}^{7}$ symmetry are insensitive to homonuclear dipole-dipole couplings, to first order.

The behavior of the isotropic chemical shift terms under $R 18_{1}^{7}$ symmetry is shown in Figure 6(c). None of the three components with $m=0$ and $\mu=\{-1,0,1\}$ pass through the barrier. This indicates that sequences with $R 18_{1}^{7}$ symmetry are insensitive to isotropic chemical shifts, to first order.

One may conclude that $R 18_{1}^{7}$ sequences accomplish recoupling of the CSA interactions, compensated to first order for homonuclear DD couplings and isotropic chemical shifts.

\subsection{Higher-Order Selection Rules}

In many cases, the first-order Magnus term (the average Hamiltonian) is not a sufficiently good approximation to the effective Hamiltonian of a pulse sequence. Although the higher-order correction terms $\overline{\mathcal{H}}^{(2)}, \overline{\mathcal{H}}^{(3)}, \ldots$ are more complicated than the first-order terms, they are also subject to selection rules in the case of pulse sequence symmetry.

By analogy with equation (25), the second-order term $\overline{\mathcal{H}}^{(2)}$ may be written as a superposition of many rotational components:

$$
\overline{\mathcal{H}}^{(2)}\left(t^{0}\right)=\sum_{l_{2} m_{2} \lambda_{2} \mu_{2}} \sum_{l_{1} m_{1} \lambda_{1} \mu_{1}} \overline{\mathcal{H}}_{l_{2} m_{2} \lambda_{2} \mu_{2}, l_{1} m_{1} \lambda_{1} \mu_{1}}^{(2)}\left(t^{0}\right)
$$

where each component is given by

$$
\begin{aligned}
& \overline{\mathcal{H}}_{l_{2} m_{2} \lambda_{2} \mu_{2}, l_{1} m_{1} \lambda_{1} \mu_{1}}^{(2)}\left(t^{0}\right) \\
& \quad=(2 \mathrm{i} T)^{-1} \int_{t^{0}}^{t^{0}+T} \mathrm{~d} t_{2} \int_{t^{0}}^{t_{2}} \mathrm{~d} t_{1}\left[\mathcal{H}_{l_{2} m_{2} \lambda_{2} \mu_{2}}\left(t_{2}\right), \mathcal{H}_{l_{1} m_{1} \lambda_{1} \mu_{1}}\left(t_{1}\right)\right]
\end{aligned}
$$

The horrifying complexity is moderated by the following selection rules:

For $C N_{n}^{v}$ sequences, the following selection rule applies:

$$
\overline{\mathcal{H}}_{l_{2} m_{2} \lambda_{2} \mu_{2}, l_{1} m_{1} \lambda_{1} \mu_{1}}^{(2)}=0 \text { if }\left\{\begin{array}{l}
m_{2} n-\mu_{2} v \neq N Z \\
\text { AND } \\
m_{1} n-\mu_{1} v \neq N Z \\
\text { AND } \\
\left(m_{2}+m_{1}\right) n-\left(\mu_{2}+\mu_{1}\right) v \neq N Z
\end{array}\right.
$$

where $Z$ is any integer, including zero.

For $R N_{n}^{v}$ sequences, the following selection rule applies:

$$
\overline{\mathcal{H}}_{l_{2} m_{2} \lambda_{2} \mu_{2}, l_{1} m_{1} \lambda_{1} \mu_{1}}^{(2)}=0 \text { if }\left\{\begin{array}{l}
m_{2} n-\mu_{2} v \neq \frac{N}{2} Z_{\lambda_{2}} \\
\mathrm{AND} \\
m_{1} n-\mu_{1} v \neq \frac{N}{2} Z_{\lambda_{1}} \\
\mathrm{AND} \\
\left(m_{2}+m_{1}\right) n-\left(\mu_{2}+\mu_{1}\right) v \neq \frac{N}{2} Z_{\lambda_{2}+\lambda_{1}}
\end{array}\right.
$$

where $Z_{\lambda_{2}}$ is an integer with the same parity as $\lambda_{2}, Z_{\lambda_{1}}$ is an integer with the same parity as $\lambda_{1}$, and $Z_{\lambda_{2}+\lambda_{1}}$ is an integer with the same parity as $\lambda_{2}+\lambda_{1}$.

These selection rules are proved in Refs. ${ }^{45,60}$

Although the second-order selection rules do not usually allow the elimination of all undesirable terms, they do greatly reduce the labor required to evaluate the terms. For example, in general, there are 208 components to the $\overline{\mathcal{H}}^{(2)}$ term involving commutators of dipole-dipole coupling and CSA interactions. In the presence of a $R 14_{2}^{6}$ sequence, only 16 of these are symmetry-allowed.

The R-sequence selection rule equation (32) is more restrictive than that for $\mathrm{C}$-sequences equation (31). As a result, Rsequences tend to have smaller numbers of higher-order terms than $\mathrm{C}$-sequences, which often translates into improved performance.

The second-order selection rules allow a straightforward count of the number of symmetry-allowed terms of a particular type. Higher-order term counts may be used to get a feel for the qualitative performance of a particular symmetry, without performing detailed calculations. ${ }^{\mathbf{4 1 , 4 5}}$ However, this rather crude criterion should not be relied on completely.

It is possible to extend these second-order selection rules to arbitrarily high orders. ${ }^{45}$

\subsection{Scaling Factors}

The selection rules provide information on which terms are symmetry-forbidden. However, they provide no information on the magnitude of the symmetry-allowed terms.

In general, the form of a symmetry-allowed first-order term is as follows:

$$
\overline{\mathcal{H}}_{l m \lambda \mu}^{\Lambda}\left(t^{0}\right)=\kappa_{l m \lambda \mu}\left[A_{l m}^{\Lambda}\right]^{R} \exp \left\{-\mathrm{i} m\left(\alpha_{R L}^{0}-\omega_{r} t^{0}\right)\right\} T_{\lambda \mu}^{\Lambda}
$$

where $\left[A_{\Lambda}^{l m}\right]^{R}$ is a component of the spatial tensor of interaction $\Lambda$, expressed in the rotor-fixed frame $R$, and $T_{\lambda \mu}^{\Lambda}$ is a component of the spin tensor of interaction $\Lambda$, expressed in the laboratory frame. The factor $\kappa_{l m \lambda \mu}$ is called the scaling factor of the pulse sequence, and depends upon the symmetry class, as well as the detailed structure of the basic element upon which the pulse sequence is built. 
The scaling factor may be a complex number. Its magnitude is always less than one, which indicates that the suppression of unwanted terms is always accompanied by a reduction in the magnitude of the symmetry-allowed terms.

\subsubsection{Scaling Factor Formulae}

General formulae for the scaling factors are given in Ref. ${ }^{\mathbf{6 0}}$ In the case of $C N_{n}^{\nu}$ sequences, the scaling factor for a symmetry-allowed term $\{l, m, \lambda, \mu\}$ is given by

$$
\begin{aligned}
& \kappa_{l m \lambda \mu}= \\
& \tau^{-1} d_{m 0}^{l}\left(\beta_{R L}\right) \int_{0}^{\tau} \mathrm{d} t d_{\mu 0}^{\lambda}\left(-\beta_{\mathrm{rf}}(t)\right) \exp \left\{\mathrm{i}\left(\mu \gamma_{\mathrm{rf}}(t)+m \omega_{r} t\right)\right\}
\end{aligned}
$$

where $\tau=n \tau_{r} / N$ is the duration of a basic element $\mathcal{C}, \tau_{r}=$ $\left|2 \pi / \omega_{r}\right|$ is a rotor period, and $\beta_{\mathrm{rf}}(t)$ and $\gamma_{\mathrm{rf}}(t)$ are rf Euler angles [equation (6)]. The basic element $C$ is assumed to extend from time $t=0$ to $t=\tau$. by

In the case of $R N_{n}^{v}$ sequences, the scaling factor is given

$$
\begin{aligned}
\kappa_{l m \lambda \mu} & =\tau^{-1} d_{m 0}^{l}\left(\beta_{R L}\right) \\
& \times \int_{0}^{\tau} \mathrm{d} t d_{\mu 0}^{\lambda}\left(-\beta_{\mathrm{rf}}(t)\right) \exp \left\{\mathrm{i}\left(\mu \gamma_{\mathrm{rf}}(t)-\mu \frac{\pi v}{N}+m \omega_{r} t\right)\right\}
\end{aligned}
$$

There is an extra complex factor in this case, since the first element of a $R N_{n}^{v}$ sequence is not the same as the basic element.

The scaling factor is calculated most readily if the basic elements of the pulse sequence do not contain any rf phase shifts, or if the phase shifts are integer multiples of $180^{\circ}$. This is the case of amplitude-modulated basic elements. For example, $\mathcal{C}=270_{0} 180_{180} 90_{0}$ is an amplitude-modulated basic element, while $\mathcal{R}=90_{-45} 90_{45} 90_{-45}$ is a phase-modulated basic element. In the case of amplitude-modulated basic elements, the rf Euler angles are given by

$$
\begin{aligned}
& \beta_{\mathrm{rf}}(t)=\int_{0}^{t} \mathrm{~d} t^{\prime} \omega_{\text {nut }}\left(t^{\prime}\right) \\
& \gamma_{\mathrm{rf}}(t)=\pi / 2
\end{aligned}
$$

where $\omega_{\text {nut }}$ is the amplitude of the rf field, expressed as a nutation frequency $\left(180^{\circ}\right.$ phase shifts may be taken into account by reversing the sign of $\left.\omega_{\text {nut }}\right)$.

The calculation of the scaling factor is more difficult if the basic element is phase modulated. General formulae are given in Ref. ${ }^{60}$ Andreas Brinkmann has written a Mathematica ${ }^{\mathbf{6 3}}$ program which provides the scaling factors of general sequences of rectangular pulses. The program is available on the web (http://www.soton.ac.uk/ mhl).

\subsubsection{Physical Interpretation}

The formulae for the scaling factor, equations (34) and (35) contain three factors. The first term, $d_{m 0}^{l}\left(\beta_{R L}\right)$ is purely spatial, and concerns the transformation of spatial rank-l tensors by the physical rotation of the sample. This term depends on the tilt of the rotation axis with respect to the magnetic field. The second term $d_{\mu 0}^{\lambda}\left(-\beta_{\mathrm{rf}}(t)\right)$ corresponds to the time-dependent transformation of rank- $\lambda$ spin operators by the rf field. The third term (the complex exponential factor) takes into account the physical rotation of the sample within the duration of each pulse sequence element. The formula for the R-sequence scaling factor, equation (35), contains a further phase factor, which arises because the first element of a $\mathrm{R}$-sequence is phase shifted with respect to the basic element $\mathcal{R}$.

In general, it is desirable that the magnitude of $\kappa_{l m \lambda \mu}$ is as large as possible. It helps if one develops a physical feel of how $\kappa_{l m \lambda \mu}$ is generated.

In most cases, the angle $\beta_{R L}$ is equal to the magic angle, so that the first term $d_{m 0}^{l}\left(\beta_{R L}\right)$ is not open to manipulation (an exception is provided by the 'zero-field in high-field' pulse sequences, where the choice of the spinning angle is an important element of the pulse sequence design).

In many cases, the symmetry number $N$ is much larger than $n$. In this case, the duration of each element is much less than a rotor period, and it is possible to ignore the rotation of the sample during the individual rf elements, as a first approximation. This corresponds to the neglect of the $m \omega_{r} t$ terms in equations (34) and (35). This is called the quasi-static approximation.

In the quasi-static limit, only the rf rotations affect the accumulating time average of $T_{\lambda \mu}$ spin operators. For example, consider the problem of double-quantum recoupling. The relevant Wigner element in this case is $d_{20}^{2}\left(\beta_{\mathrm{rf}}\right)$, which is proportional to $\sin ^{2}\left(\beta_{\mathrm{rf}}\right)$. This term is always positive, and is maximized at $\beta_{\mathrm{rf}}=90^{\circ}$ and $\beta_{\mathrm{rf}}=270^{\circ}$. It follows that the main contributions to the scaling factor are obtained when the Euler angle $\beta_{\mathrm{rf}}$ is close to the values $90^{\circ}$ or $270^{\circ}$. At the same time, the Euler angle $\gamma_{\mathrm{rf}}$ should be kept fixed. As shown in equation (36), this may be ensured by using an amplitudemodulated basic element.

The simplest cyclic element is $\mathcal{C}=360_{0}$. The rotation under this pulse takes the Euler angle $\beta_{\mathrm{rf}}$ linearly from 0 to $360^{\circ}$. Double-quantum excitation is accomplished near the 'hot spots' at $\beta_{\mathrm{rf}}=90^{\circ}$ and $\beta_{\mathrm{rf}}=270^{\circ}$. No doublequantum excitation is achieved, on the other hand, at the 'cold spots' $\beta_{\mathrm{rf}}=0$ and $\beta_{\mathrm{rf}}=180^{\circ}$. The element $\mathcal{C}=360_{0}$ spends equal time near the 'hot' and 'cold' spots. Nevertheless, the appropriate Wigner element is always positive, so the scaling factor is satisfactory $\left(\left|\kappa_{2122}\right|=0.157\right.$ for the case of $C 7_{2}^{1}$ symmetry).

The scaling factor may be improved by turning the rf field off near the 'hot spots' $\beta_{\mathrm{rf}}=90^{\circ}$ and $\beta_{\mathrm{rf}}=270^{\circ}$, allowing the double-quantum operators more time to accumulate. An example of this technique is given by the sequence

$$
\mathcal{C}=90_{0}-\tau_{\omega}-180_{0}-\tau_{\omega}-90_{0}
$$

where the 'window' duration $\tau_{\omega}$ is selected so that the overall element occupies an interval $\tau=n \tau_{r} / N$. In the limit of infinitely strong and short pulses, the element in equation (37) provides a greatly improved scaling factor of $\left|\kappa_{2122}\right|=0.308$ for the case of $C 7_{2}^{1}$ symmetry. Although the scaling factor may be increased by introducing windows, this method has not been used very often, perhaps because the strong rf pulses make the problem of proton decoupling more acute (see below).

In practice, it is common to use elements which are locally compensated for rf inhomogeneity. This improves the robustness of the pulse sequence to $\mathrm{rf}$ amplitude deviations. The original version of the $\mathrm{C} 7$ pulse sequence used the locallycompensated element $\mathcal{C}=360_{0} 360_{180}$ in a $C 7_{2}^{1}$ symmetry 
scheme. ${ }^{36}$ In this case, each of the double-quantum 'hot spots' at $\beta_{\mathrm{rf}}=90^{\circ}$ and $\beta_{\mathrm{rf}}=270^{\circ}$ are traversed twice during each element. The scaling factor of $\left|\kappa_{2122}\right|=0.155$ is almost the same as for the simple element $\mathcal{C}=360_{0}$, which has $\left|\kappa_{2122}\right|=$ 0.157 . The values are slightly different because of the rotation of the sample during the rf element.

In the quasi-static limit, the efficiency of double-quantum recoupling is greatly reduced if the Euler angle $\gamma_{\mathrm{rf}}$ is allowed to vary. For example, the scaling factor for the phase-modulated element $\mathcal{C}=360_{0} 360_{120} 360_{240}$ is given by $\left|\kappa_{2122}\right|=0.047$ in the case of $C 7_{2}^{1}$ symmetry.

If the values of $N$ and $n$ are comparable, the quasi-static approximation breaks down. In this case, it is necessary to think much more carefully about how the physical rotation of the sample and the spin rotations combine. Amplitudemodulated elements are no longer optimal as far as the scaling factor is concerned. For example, consider the symmetry $C 7_{5}^{1}$, which provides similar selection rules to $C 7_{2}^{1}$ (except that the $\{l, m, \lambda, \mu\}=\{2, \pm 1,2, \mp 2\}$ terms are selected, rather than $\{2, \pm 1,2, \pm 2\})$. In the case of $C 7_{5}^{1}$ symmetry, the scaling factor for the allowed term $\{l, m, \lambda, \mu\}=\{2,1,2,-2\}$ is given by $\left|\kappa_{212-2}\right|=0.064$ for the amplitude-modulated standard element $\mathcal{C}=360_{0} 360_{180}$. This low scaling factor is due to the considerable rotation of the sample during the $\mathcal{C}$ element, leading to a strong variation of the $\omega_{r} t$ term in equation (34). ${ }^{39,40}$ The scaling factor may be greatly improved by using the phase-modulated element $\mathcal{C}=360_{0} 360_{120} 360_{240}$. For the case of $C 7_{5}^{1}$ symmetry, one obtains the value $\left|\kappa_{212-2}\right|=$ $0.144 .{ }^{39}$ The improvement in scaling factor arises because the modulations of the Euler angle $\gamma_{\mathrm{rf}}$ due to the rf phase shifts compensate the modulation of the $\omega_{r} t$ term, to a good approximation. Note that the phase shifts must be performed in the correct sense: The scaling factor for the element $\mathcal{C}=360_{0} 360_{240} 360_{120}$, in the case of $C 75$ symmetry, is only $\left|\kappa_{212-2}\right|=0.044$.

In some cases, pulse sequences work even if the scaling factor is very small. For example, the most commonlyused version of the RFDR pulse sequence ${ }^{\mathbf{3 3 , 3 4}}$ employs XY phase cycling and conforms to $R 4_{4}^{1}$ symmetry. This symmetry is appropriate for zero-quantum DD recoupling, since it allows the homonuclear DD terms $\{l, m, \lambda, \mu\}=\{2, \pm 1,2,0\}$ and $\{2, \pm 2,2,0\}$, while suppressing other DD terms and all chemical shift terms. However, the first reports of this pulse sequence used an $\mathcal{R}$ element consisting of a strong $180^{\circ}$ pulse followed by a 'window' in which the rf field is turned off. This is a puzzling choice of basic element, since the scaling factors for all symmetry-allowed DD terms vanish if the rf pulses are infinitely short. In fact, this pulse sequence reasonably well because the rf pulses always have a finite duration, so that the scaling factor is small but finite. In addition, $J$-couplings and higher-order effects play a role. Recent implementations of RFDR take a more sophisticated approach. ${ }^{\mathbf{6 4 , 6 5}}$

\subsubsection{Vanishing Scaling Factors}

In many cases, the scaling factors for some symmetryallowed terms are deliberately set to zero. For example, the symmetry $C 7_{2}^{1}$ allows the isotropic chemical shift term $\{l, m, \lambda, \mu\}=\{0,0,1,0\}$. Pulse sequences with this symmetry are therefore vulnerable to isotropic shift variations. The basic elements $\mathcal{C}=360_{0}, 360_{0} 360_{180}$ and $90_{0} 360_{180} 270_{0}$ set the scaling factor $\kappa_{0010}$ to zero, compensating these pulse sequences for isotropic chemical shifts or resonance offset effects. $R$ symmetries such as $R 14_{2}^{6}$ do not need this precaution, since all isotropic chemical shift terms are symmetry-forbidden in this case.

Another example is provided by the MELODRAMA sequence for homonuclear dipolar recoupling. ${ }^{\mathbf{6 6}}$ Some versions of this sequence are based on $C 4_{1}^{1}$ symmetry. In these cases, the isotropic shift term $\{l, m, \lambda, \mu\}=\{0,0,1,0\}$ is symmetryallowed, but may be suppressed by using basic elements of the form $\mathcal{C}=(360 k)_{0}$, where $k$ is an integer. Other versions of the MELODRAMA sequence ${ }^{66}$ have $R 4_{1}^{1}$ symmetry, which suppresses all isotropic shift terms without extra precautions. None of these symmetries, however, remove all of the CSA terms.

The recently-described C-REDOR sequences ${ }^{67}$ are remarkable examples of how the basic element may be designed so as to destroy undesirable symmetry-allowed terms. The main variants of C-REDOR sequences are based upon $C 3_{3}^{1}$ and $C 7_{7}^{1}$ symmetry. These symmetries allow CSA terms of the form $\{l, m, \lambda, \mu\}=\{2, \pm 2,1,0\}$ and $\{2, \pm 1,1,0\}$, the isotropic shift term $\{0,0,1,0\}$, and also the homonuclear DD coupling terms $\{2, \pm 2,2,0\}$ and $\{2, \pm 1,2,0\}$. However, by choosing a basic element $\mathcal{C}=90_{0} 360_{180} 270_{0}$, the scaling factors of all terms vanish, except for those belonging to the CSA terms $\{2, \pm 1,1,0\}$. These sequences may therefore be used to selectively recouple CSA terms while suppressing the isotropic chemical shifts and homonuclear DD couplings. C-REDOR has been applied to the selective recoupling of heteronuclear DD interactions, ${ }^{67}$ which behave in the same way as the CSA terms, if the rf field is resonant with only one spin species (see below).

The vanishing scaling factors of certain terms under specific pulse sequences is probably due to additional symmetries. This is a promising area for further theory.

\subsection{Orientation Dependence}

In most cases, the magnitudes of the recoupled spin interactions depend on the orientation of the molecular reference frame with respect to the rotor. The only exceptions are when isotropic spin interactions such as the $J$-coupling or the isotropic chemical shift are selected, or in the special case of 'zero-field in high-field' experiments. ${ }^{\mathbf{2 8 , 2 9}}$

The orientation-dependence of the recoupled spin interactions creates both problems and opportunities. A powerful feature of the symmetry theory is that it allows the form of the orientation-dependence to be manipulated qualitatively by choosing certain combinations of symmetry numbers.

The recoupled spin Hamiltonian in equation (33) is proportional to the spatial tensor component in the rotor-fixed frame, defined through the transformation chain

$$
\left[A_{l m}^{\Lambda}\right]^{R}=\sum_{m^{\prime \prime}, m^{\prime}}\left[A_{l m^{\prime \prime}}^{\Lambda}\right]^{P} D_{m^{\prime \prime} m^{\prime}}^{l}\left(\Omega_{P M}^{\Lambda}\right) D_{m^{\prime} m}^{l}\left(\Omega_{M R}\right)
$$

Here $\left[A_{l m}^{\Lambda}\right]^{P}$ are components of the spatial tensor, expressed in its own principal axis frame. The expression in equation (38) depends on the three Euler angles $\Omega_{M R}=$ $\left\{\alpha_{M R}, \beta_{M R}, \gamma_{M R}\right\}$ defining the orientation of the molecular reference frame with respect to the rotor frame. In general, the recoupled spin Hamiltonian is anisotropic, and depends upon all three orientational Euler angles. 


\subsection{1 $\gamma$-Encoding}

Suppose that a particular set of quantum numbers $\{l, m, \lambda, \mu\}$ is symmetry-allowed in the average Hamiltonian. The spatial Wigner element has the following form:

$$
D_{m^{\prime} m}^{l}\left(\Omega_{M R}\right)=\exp \left\{-\mathrm{i} m^{\prime} \alpha_{M R}\right\} d_{m^{\prime} m}^{l}\left(\beta_{M R}\right) \exp \left\{-\mathrm{i} m \gamma_{M R}\right\}
$$

This may be combined with equation (33) to show how a single recoupled term depends upon the Euler angle $\gamma_{M R}$, the time point $t^{0}$ at which the symmetry-based pulse sequence starts, and the rotor Euler angle $\alpha_{R L}^{0}$ at the time point $t=0$ :

$$
\begin{aligned}
\overline{\mathcal{H}}_{l m \lambda \mu}^{\Lambda}\left(\gamma_{M R}, t^{0}, \alpha_{R L}^{0}\right)= & \overline{\mathcal{H}}_{\operatorname{lm} \lambda \mu}^{\Lambda}(0,0,0) \\
& \times \exp \left\{-\mathrm{i} m\left(\gamma_{M R}+\alpha_{R L}^{0}-\omega_{r} t^{0}\right)\right\}
\end{aligned}
$$

The three parameters $\gamma_{M R}, t^{0}$ and $\alpha_{R L}^{0}$ may be included in a single complex phase factor.

It follows that only the phase, and not the magnitude, of the single term $\overline{\mathcal{H}}_{l m \lambda \mu}^{\Lambda}$ depends on the orientational Euler angle $\gamma_{M R}$. This property is termed $\gamma$-encoding of the recoupled term $\overline{\mathcal{H}}_{\operatorname{lm} \lambda \mu}^{\Lambda}$.

In general, there are several symmetry-allowed terms $\overline{\mathcal{H}}_{l m \lambda \mu}^{\Lambda}$ with the same value of $l, \lambda$ and $\mu$ but different values of $m$. Each of these terms contains the same spin operator $T_{\lambda \mu}^{\Lambda}$, but with a different spatial coefficient. These multiple coefficients interfere in a complicated way. As a result, the complete recoupled spin Hamiltonian is usually not $\gamma$-encoded.

It is possible to maintain $\gamma$-encoding of the entire recoupled Hamiltonian, by imposing a one-to-one correspondence between the symmetry-allowed spatial quantum numbers $\{l, m\}$ and the symmetry-allowed spin quantum numbers $\{\lambda, \mu\}$. This prevents the interference of terms with the same value of $\mu$ but different values of $m$.

For example, consider the symmetry $C 6_{2}^{1}$. This leads to double-quantum recoupling of the DD terms, with suppression of all CSA terms to first order. The symmetry-allowed DD terms have the quantum numbers $\{2,-2,2,2\},\{2,-1,2,-2\}$, $\{2,1,2,2\}$ and $\{2,2,2,-2\}$. The average Hamiltonian is not $\gamma$-encoded, since each spin term $\mu=2$ is associated with two spatial components, $m=-2$ and $m=1$.

If the symmetry number $N$ is increased from 6 to 7, the symmetry $C 7_{2}^{1}$ is generated, which only allows the DD terms $\{2,-1,2,-2\}$ and $\{2,1,2,2\}$. Since each spin component is now associated with only a single spatial component, the recoupled spin Hamiltonian is $\gamma$-encoded. Some of the success of the $\mathrm{C} 7$ pulse sequence and its variants may be attributed to the $\gamma$-encoding of the average Hamiltonian. For example, the theoretical maximum efficiency of double-quantum filtering experiments is usually increased by around $20 \%$ by using a $\gamma$-encoded pulse sequence. ${ }^{\mathbf{3 6}}$ In addition, $\gamma$-encoded pulse sequences enjoy much greater freedom in the relative timing of the pulse sequence and the sample rotation (see below).

The orientation-dependence of $\gamma$-encoded and non- $\gamma$ encoded sequences are contrasted in Figure 7. For $\gamma$-encoded sequences, the magnitude of the recoupled Hamiltonian is cylindrically symmetrical around the sample rotation axis.

\subsubsection{Non- $\gamma$-Encoded Sequences}

Although $\gamma$-encoding is generally a positive feature, it does have some disadvantages. As explained above, $\gamma$-encoding is (a)

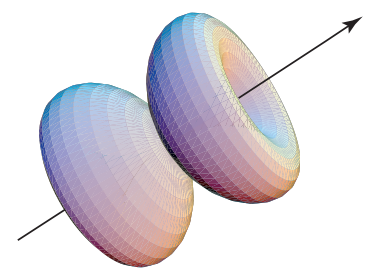

(b)

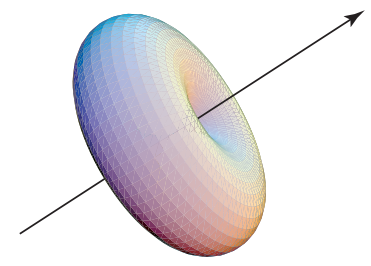

(c)

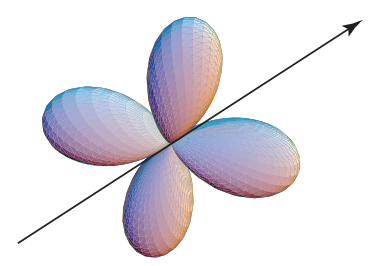

Figure 7 Polar plots showing the orientation-dependence of different dipolar recoupling sequences with respect to the magic-angle rotation axis (solid arrow). The direction of the external field is vertical. In each case, the magnitude of the recoupled Hamiltonian for a given orientation of the internuclear vector is proportional to the distance from the origin to the surface, in the direction of the vector. (a) $\gamma$-encoded recoupling of $m= \pm 1$ terms; (b) $\gamma$-encoded recoupling of $m= \pm 2$ terms; (c) non- $\gamma$-encoded recoupling

generally imposed by eliminating some components of the average Hamiltonian. This enhanced selectivity is generally accompanied by a reduction in the overall magnitude of the recoupled Hamiltonian. In some cases, for example in longrange distance measurements, this is a disadvantage, since the recoupled interactions compete with relaxation and other loss processes. As a result, non- $\gamma$-encoded pulse sequences are sometimes preferable if the largest possible recoupled Hamiltonian is required, and its orientation-dependence is of lesser importance.

The complicated orientation-dependence of non- $\gamma$-encoded sequences may also be put to good use. Spiess and coworkers $^{\mathbf{6 8 , 6 9}}$ have shown that the orientation-dependence generates characteristic signal modulations in certain twodimensional experiments. These modulations may be matched with simulations in order to obtain structural information such as internuclear distances. In these applications, the recoupling pulse sequence must not be $\gamma$-encoded.

A further disadvantage of $\gamma$-encoding is that the construction of compensated supercycles is more difficult.

Some recoupled Hamiltonians cannot be $\gamma$-encoded within the framework of the symmetry theory described here. For example, consider the problem of zero-quantum recoupling in the presence of MAS. Since the selection rules are invariant to an overall sign change, it is not possible to allow terms of the form $\{l, m, \lambda, \mu\}=\{2, m, 2,0\}$ without also allowing terms of the form $\{2,-m, 2,0\}$. Since the $\mu=0$ term is associated with two spatial terms $\pm m$, the resulting Hamiltonian is not $\gamma$-encoded. If desired, a $\gamma$-encoded zero-quantum DD Hamiltonian may be generated by rotational resonance, ${ }^{\mathbf{7 0}-72}$ which 
is based on different principles (see Rotational Resonance in Biology, Volume 7).

\section{$2.13 .3 \quad \beta$-Dependence}

Consider the $\gamma$-encoded recoupling of dipole-dipole interactions between two spins $j$ and $k$. Since the DD interaction is symmetrical, the tensor components $\left[A_{2 m}^{j k}\right]^{P}$, written in the principal axis system of the DD interaction, vanish for the case $m \neq 0$. From equation (33), the recoupled DD interaction between spins $j$ and $k$ has the form

$$
\begin{aligned}
\overline{\mathcal{H}}_{2 m 2 \mu}^{j k}\left(t^{0}\right)= & \kappa_{2 m 2 \mu}\left[A_{20}^{j k}\right]^{P} d_{0 m}^{2}\left(\beta_{P R}^{j k}\right) \\
& \times \exp \left\{-\mathrm{i} m\left(\gamma_{P R}^{j k}+\alpha_{R L}^{0}-\omega_{r} t^{0}\right)\right\} T_{2 \mu}^{j k}
\end{aligned}
$$

Here $\beta_{P R}^{j k}$ and $\gamma_{P R}^{j k}$ are Euler angles relating the principal axis frame of the DD interaction to the rotor-fixed frame. For example, $\beta_{P R}^{j k}$ is the angle subtended by the internuclear $j \rightarrow k$ vector and the rotation axis of the sample.

If the sequence implements $\gamma$-encoded recoupling, then equation (41) contains the entire orientation-dependence of the $T_{2 \mu}^{j k}$ spin operator. As may be seen, the magnitude of the recoupled Hamiltonian depends on $\beta_{P R}^{j k}$ according to the reduced Wigner function $d_{0 m}^{2}\left(\beta_{P R}^{j k}\right)$.

The relevant functions have the following form:

$$
\begin{aligned}
d_{00}^{2}(\beta) & =\frac{1}{2}\left(3 \cos ^{2} \beta-1\right) \\
d_{0 \pm 1}^{2}(\beta) & = \pm \sqrt{\frac{3}{8}} \sin 2 \beta \\
d_{0 \pm 2}^{2}(\beta) & =\sqrt{\frac{3}{8}} \sin ^{2} \beta
\end{aligned}
$$

The type of orientation-dependence may be selected by choosing the $m$-value of the symmetry-allowed component.

For example, consider the double-quantum recoupling sequences with symmetry $C 7_{2}^{1}$. As discussed above, this symmetry implements $\gamma$-encoded recoupling with selection of the $\{l, m, \lambda, \mu\}=\{2,1,2,2\}$ and $\{2,-1,2,-2\}$ terms. Since the $m= \pm 1$ terms are symmetry-allowed, the magnitude of the recoupled Hamiltonian is proportional to $d_{01}^{2}\left(\beta_{P R}^{j k}\right)$, and hence depends upon orientation according to the function $\sin \left(2 \beta_{P R}^{j k}\right)$. The recoupling effect is maximized at the angles $\beta_{P R}^{j k}=45^{\circ}$ and $135^{\circ}$, and vanishes at the orientations $\beta_{P R}^{j k}=0$ or $180^{\circ}$ (internuclear vector along the spinning axis) and also $\beta_{P R}^{j k}=$ $90^{\circ}$ (internuclear vector perpendicular to the spinning axis). A sketch of the orientation dependence of the $C 7_{2}^{1}$ recoupled Hamiltonian is shown in Figure 7(a).

The symmetry $C 8_{3}^{1}$, on the other hand, implements $\gamma$-encoded double-quantum recoupling with selection of the terms $\{l, m, \lambda, \mu\}=\{2,-2,2,2\}$ and $\{2,2,2,-2\}$. In this case, the magnitude of the recoupled Hamiltonian is proportional to $d_{02}^{2}\left(\beta_{P R}^{j k}\right)$, and hence depends upon orientation according to the function $\sin ^{2}\left(\beta_{P R}^{j k}\right)$. In this case, the recoupling effect is maximized at the angle $\beta_{P R}^{j k}=\pi / 2$ (internuclear vector perpendicular to the spinning axis) and vanishes for the angles $\beta_{P R}^{j k}=0$ and $\pi$ (internuclear vector parallel to the spinning axis). A sketch of the orientation dependence of the $C 8_{3}^{1}$ recoupled Hamiltonian is shown in Figure 7(b).
These orientation-dependences may be exploited in the magic-angle spinning of oriented materials. ${ }^{\mathbf{5 5 , 5 6 , 7 3 - 7 5}}$ Glaubitz has proposed a series of different recoupling experiments, in order to deduce the angles subtended by internuclear vectors and the spinning axis with a minimum of ambiguity. ${ }^{75}$ This should enhance the molecular structural information which may be obtained from the magic-angle spinning NMR of oriented samples.

The symmetry-aided manipulation of orientation-dependence may also be useful in the NMR of disordered samples such as powders. It is possible to gain information on the relative orientation of pairs of interactions by correlating them with each other using multiple-dimensional NMR methods. ${ }^{43,44,76-81}$ It should be possible to enhance the information content of such experiments, and resolve ambiguities, by using combinations of recoupling symmetries applied in different time intervals of a multidimensional experiment. At the moment, such experiments are still in the planning stage.

\subsection{Phase-Time Relationships}

The spin tensors transform in the following way when rotated about the $z$-axis:

$$
\exp \left\{-\mathrm{i} \phi I_{z}\right\} T_{\lambda \mu}^{\Lambda} \exp \left\{-\mathrm{i} \phi I_{z}\right\}=T_{\lambda \mu}^{\Lambda} \exp \{-\mathrm{i} \mu \phi\}
$$

This equation may be combined with equation (40) to obtain the dependence of a recoupled Hamiltonian term on the orientational angles $\gamma_{M R}$ and $\alpha_{R L}^{0}$, the rf phase $\phi$, and the initial time point $t^{0}$ :

$$
\begin{aligned}
& \overline{\mathcal{H}}_{l m \lambda \mu}^{\Lambda}\left(\gamma_{M R}, t^{0}, \alpha_{R L}^{0}, \phi\right)=\overline{\mathcal{H}}_{l m \lambda \mu}^{\Lambda}(0,0,0,0) \\
& \times \exp \left\{-\mathrm{i}\left[m\left(\gamma_{M R}+\alpha_{R L}^{0}-\omega_{r} t^{0}\right)+\mu \phi\right]\right\}
\end{aligned}
$$

As discussed in Ref. ${ }^{\mathbf{8 2}}$, this relationship may be used to remove the dependence of the recoupled Hamiltonian on the initial time point $t^{0}$. The desired effect is achieved for the case $\mu \neq 0$ by shifting the overall rf phase according to the formula:

$$
\phi=\frac{m}{\mu} \omega_{r} t^{0}
$$

If this is done, then the average Hamiltonian term $\overline{\mathcal{H}}_{l m \lambda \mu}^{\Lambda}$ is independent of the initial time point $t^{0}$ at which the pulse sequence starts. Equation (45) is called a phase-time relationship.

In general, the recoupled Hamiltonian contains many symmetry-allowed terms, which may have different ratios of $m$ and $\mu$. If that is the case, then the formula in equation (45) cannot be implemented simultaneously for all of them, and it is impossible to compensate the full recoupled Hamiltonian for a change in the timing by a shift in the rf phase. This is clearly the case if the sequence is not $\gamma$-encoded.

If the sequence is $\gamma$-encoded, and if all the symmetryallowed terms have the same ratio $m / \mu$, then the pulse sequence may be applied at arbitrary time points in a pulse sequence, providing the overall $\mathrm{rf}$ phase is adjusted according to equation (45). This is an important practical principle of $\gamma$-encoded recoupling sequences, which has been applied in many different experimental contexts. ${ }^{39,60,79,82-84}$ 
A typical application is two-dimensional double-quantum spectroscopy. The pulse sequence consists of a preparation period, which may consist of a $C N_{n}^{v}$ recoupling sequence, followed by a variable evolution interval $t_{1}$, followed by a mixing interval, which might be a second $C N_{n}^{v}$ sequence, followed by signal detection.

In solution NMR, it is possible to vary the evolution interval $t_{1}$ in arbitrarily small steps, in order to obtain a sufficiently large spectral bandwidth in the double-quantum dimension. This is because the operation of the preparation and mixing sequences is independent of the times at which they are applied.

In magic-angle-spinning solid-state NMR, on the other hand, the situation is completely different, since the rotation of the sample modulates the spin Hamiltonian during the pulse sequence. In general, the average Hamiltonian generated by the second recoupling sequence depends on the time at which it is applied, and hence on the interval $t_{1}$. As a result, multi-dimensional experiments often display strong periodic modulations in the indirectly-detected dimension, which are due to the rotation of the sample during $t_{1}$ (see DoubleQuantum NMR Spectroscopy of Dipolar Coupled Spins Under Fast Magic-Angle Spinning). It is easy to misinterpret these modulations as being due to the evolution of coherences in the interval $t_{1}$.

If the sequence is not $\gamma$-encoded, the only way to avoid these orientational modulations is to restrict $t_{1}$ to be integer multiples of a rotor period, so that the sample has the same orientation at the beginning and end of the evolution interval. However, this is a very strong restriction which in many cases greatly cramps the spectral width.

However, if the sequence is $\gamma$-encoded, and if all symmetry-allowed terms have the same ratio $m / \mu$, then it is possible to vary the evolution interval completely freely by coupling the rf phase to the timing, according to the phase-time relationship equation (45). This allows multiple-quantum spectroscopy with an arbitrarily large bandwidth in the indirectlydetected dimension. Examples, including explicit formulae for the radio-frequency phases, are given in Refs. ${ }^{39,60,79,82-84}$

\subsection{Incomplete Cycles}

As mentioned before, the average Hamiltonian theory is strictly valid only for integer multiples of complete $C N_{n}^{v}$ or $R N_{n}^{v}$ cycles, i.e., complete multiples of $n$ rotor periods. However, in most cases, the theory seems to work very well even when the full cycles are not completed. This allows a further degree of freedom in experimental design, since it becomes possible to adjust the recoupling intervals in relatively fine time steps.

This is an important advantage in experiments which aim to measure the magnitude of recoupled interactions. A fine time resolution of the recoupling interval is often a prerequisite for obtaining a result of the desired accuracy. Fine time resolution also makes it easier to maximize signal strength in multiplequantum-filtering experiments.

There is an important complication associated with the use of incomplete recoupling cycles. In general, the pulse sequence propagator under a recoupling sequence is the product of two terms (equation (22)). The first term is the propagator for the rf field under the pulse sequence, while the second term concerns the average Hamiltonian of the internal spin interactions, which is subject to the selection rules discussed above. In the case of complete $C N_{n}^{v}$ or $R N_{n}^{v}$ sequences, the first term may be omitted, since the complete sequence behaves as a cycle, with an $\mathrm{rf}$ propagator proportional to the unity operator. However, this is not always the case for incomplete pulse sequences.

The $C N_{n}^{v}$ sequences present few problems in this respect, since the rf propagator for an incomplete $C N_{n}^{v}$ sequence is still proportional to the unity operator, providing that integer numbers of $\mathcal{C}$ elements are completed. As a result, only the average Hamiltonian must be taken into account for integer multiples of $\mathcal{C}$ elements.

Matters are more complicated for $R N_{n}^{v}$ sequences. If $q$ elements $\mathcal{R}$ are completed, the rf propagator is given by

$$
U_{\mathrm{rf}}\left(t^{0}+q \tau_{\mathcal{R}}, t^{0}\right)=\exp \left\{-\mathrm{i} 2 q \phi I_{z}\right\} \exp \left\{-\mathrm{i} q \pi I_{x}\right\}
$$

where $I_{x}$ and $I_{z}$ is the longitudinal angular momentum operator of the irradiated spins, and the phase $\phi$ is given ideally by $\phi=\pi \nu / N$. The duration of each element is $\tau_{\mathcal{R}}=n \tau_{r} / N$, where $\tau_{r}$ is a rotor cycle.

In the case of even $q$, the rf propagator leads to an additional $z$-rotation of the spin operators, which must be taken into account by a compensatory rf phase shift of subsequent $\mathrm{rf}$ pulses. This phase shift must be added to the phase shifts imposed by the phase-time relationships, described above, as well as any phase shifts required in the phase cycling procedure, used to select NMR signals passing through certain orders of spin coherence.

In the case of odd $q$, there is a rotation of the spins by an odd multiple of $\pi$ about the $x$-axis, as well as the phase rotation by $2 q \phi$. The additional $x$-rotation may be taken into account by changing the sign of subsequent $\mathrm{rf}$ phases.

The overall implementation of these multiple phase shifts and phase inversions is quite complex. Refs. ${ }^{39,60,79,82-84}$ give some practical examples with explicit formulae for the $\mathrm{rf}$ phases.

If these phase factors are taken into account correctly, it is possible to achieve fine time resolution of both the duration of the recoupling sequences and the intervals between them. In many cases, this is a decisive practical advantage.

\subsection{Supercycles}

In many cases, the performance of the recoupling sequences is enhanced by supercycling, which means repetition of the sequence with some variation of the rf phase shifts or the order of the pulse sequence elements.

The effect of supercycling is well understood on the level of the first-order average Hamiltonian. The higher order effects of supercycling, on the other hand, are currently not well researched, despite their importance. Some of the results sketched below should be regarded as preliminary. Refs. ${ }^{39,60}$ contain some relevant theory.

Most of the supercycles used so far fall into the categories summarized below:

\subsection{1 $180^{\circ}$ Phase Shift Supercycles}

If a complete $C N_{n}^{v}$ or $R N_{n}^{v}$ sequence is repeated with a $180^{\circ}$ phase shift, we get a two-step supercycle denoted $[\mathcal{S}]_{0}[\mathcal{S}]_{180}$. 
Here $[\mathcal{S}]_{\phi}$ indicates an overall phase shift of all elements in the sequence $\mathcal{S}$ by the angle $\phi$.

This supercycle cancels all components with odd values of $\mu$ in the first-order average Hamiltonian. The supercycle therefore implements an additional selection rule which is superimposed on the $C N_{n}^{v}$ or $R N_{n}^{v}$ selection rules.

For example, the symmetry $C 5_{2}^{1}$ implements $\gamma$-encoded double-quantum homonuclear recoupling, with selection of the $\{l, m, \lambda, \mu\}=\{2, \pm 1,2, \pm 2\}$ terms, similar to $C 7_{2}^{1}$. However, $C 5_{2}^{1}$ symmetry also allows CSA terms with quantum numbers $\{2, \pm 2,1, \mp 1\}$ and single-quantum DD terms with quantum numbers $\{2, \pm 2,2, \mp 1\}$. These undesirable terms all have odd values of $\mu$ and may be suppressed by building a supercycle of the form $\left[C 5_{2}^{1}\right]_{0}\left[C 5_{2}^{1}\right]_{180}$. The resulting sequence is called SPC5. ${ }^{40}$ It has the same first-order average Hamiltonian properties as $C 7_{2}^{1}$ but lower rf field requirements. ${ }^{40}$ There are other ways of achieving the same result. ${ }^{39}$

Supercycles of the form $[\mathcal{S}]_{0}[\mathcal{S}]_{180}$ may be used whenever the desired components of the first-order average Hamiltonian have even values of $\mu$. Even when this supercycle does not add to the selectivity of the first-order average Hamiltonian, it tends to reduce higher-order terms. For example, the CSA compensation of $R N_{n}^{v}$ sequences for homonuclear doublequantum recoupling is improved by using supercycles of the form $\left[R N_{n}^{\nu}\right]_{0}\left[R N_{n}^{\nu}\right]_{180} .^{\mathbf{8 5}}$

\subsection{2 $120^{\circ}$ Phase Shift Supercycles}

A higher degree of coherence order selectivity is implemented by using three-step phase cycles of the form $[\mathcal{S}]_{0}[\mathcal{S}]_{120}[\mathcal{S}]_{240}$. Supercycles of this type suppress all terms in the average Hamiltonian in which $\mu$ is not an integer multiple of 3. Since all spin interactions have $\mu$ values between 2 and -2 , this supercycle $[\mathcal{S}]_{0}[\mathcal{S}]_{120}[\mathcal{S}]_{240}$ selects the $\mu=0$ components in the average Hamiltonian, suppressing single-quantum and double-quantum terms.

This type of supercycle is useful for stabilizing zeroquantum recoupling sequences such as $R 4_{4}^{1} \cdot{ }^{\mathbf{6 5}}$

\subsubsection{Phase Inversion Supercycles}

A different effect is achieved by supercycles of the form $\mathcal{S S}^{\prime}$, where $\mathcal{S}^{\prime}$ indicates the sign reversal of all $\mathrm{rf}$ phase shifts in the sequence $\mathcal{S}$. For example, if $\mathcal{S}$ consists of a $R N_{n}^{-v}$ sequence based on the elements $\mathcal{R}=$ $90_{-45} 90_{45} 90_{-45}$ and $\mathcal{R}^{\prime}=90_{45} 90_{-45} 90_{45}$, then $\mathcal{S}^{\prime}$ consists of a $R N_{n}^{v}$ sequence based on the elements $\mathcal{R}=90_{45} 90_{-45} 90_{45}$ and $\mathcal{R}^{\prime}=90_{-45} 90_{45} 90_{-45}$.

This type of supercycle replaces first-order average Hamiltonian terms of the form $a T_{\lambda \mu}+a^{*} T_{\lambda-\mu}$ by the expression $\operatorname{Re}\{a\}\left(T_{\lambda \mu}+T_{\lambda-\mu}\right)$. The effect is to convert the phase modulation of the average Hamiltonian into an amplitude modulation.

This type of supercycle cannot be used for $\gamma$-encoded sequences, but has been used with advantage for zero-quantum recoupling sequences, which cannot be $\gamma$-encoded. ${ }^{65}$ It is used in many popular implementations of $\operatorname{REDOR}^{26,27}$ and RFDR. $^{33,34}$

\subsubsection{Combined Phase Inversion and $180^{\circ}$ Pulse Insertion Supercycles}

It is possible to combine phase inversion with the insertion of strong $180^{\circ}$ pulses, leading to supercycles of the form
$\mathcal{S} \Pi_{0} \mathcal{S}^{\prime} \Pi_{180}$, where $\Pi_{\phi}$ indicates the insertion of a strong, short, $180^{\circ}$ pulse of phase $\phi$.

If the inserted $180^{\circ}$ pulses are ideal and infinitely short, this supercycle maintains the $\gamma$-encoding of the average Hamiltonian and also deletes average Hamiltonian terms with odd values of the spin rank $\lambda$. This type of supercycle is particularly useful for improving the chemical shift compensation of a pulse sequence.

Tycko used this type of supercycle to compensate 'zerofield in high-field' experiments for chemical shifts. ${ }^{\mathbf{2 8 , 2 9}}$

In practice, $180^{\circ}$ pulse insertion supercycles are awkward to implement, especially at high spinning frequencies, because the finite duration of real $180^{\circ}$ pulses disturbs the timing of the other pulse sequence elements, breaking the pulse sequence symmetry.

\subsubsection{Combined Phase Inversion and Cyclic Permutation Supercycles}

A similar effect to the insertion of strong $180^{\circ}$ pulses is achieved by cyclically permuting a $180^{\circ}$ pulse sequence element. For example, the cyclic permutation of a $180^{\circ}$ element $\mathcal{R}$ of the phase-inverted cycle $\mathcal{S}^{\prime}$ may be denoted $\mathcal{R}^{-1} \mathcal{S}^{\prime} \mathcal{R}$, where the notation $\mathcal{R}^{-1} \mathcal{S}^{\prime}$ indicates the deletion of the element $\mathcal{R}$ from the beginning of the sequence $\mathcal{S}^{\prime}$.

For example, consider a $C 14_{4}^{5}$ sequence based on the simple element $\mathcal{C}=360_{0}$, i.e.,

$$
\begin{aligned}
\mathcal{S}= & 360_{0} 360_{128.57} 360_{257.14} 360_{25.71} 360_{154.29} 360_{282.86} 360_{51.43}- \\
& 360_{180} 360_{308.57} 360_{77.14} 360_{205.71} 360_{334.29} 360_{102.86} 360_{231.43}
\end{aligned}
$$

spanning four rotational periods. The phase inverted cycle $\mathcal{S}^{\prime}$ is as follows:

$$
\begin{aligned}
\mathcal{S}^{\prime}= & 360_{0} 360_{231.43} 360_{102.86} 360_{334.29} 360_{205.71} 360_{77.14} 360_{308.57}- \\
& 360_{180} 360_{51.43} 360_{282.86} 360_{154.29} 360_{25.71} 360_{257.14} 360_{128.57}
\end{aligned}
$$

The sequence $\mathcal{R}^{-1} \mathcal{S}^{\prime} \mathcal{R}$ derived by cyclically-permuting the element $\mathcal{R}=180_{0}$ of $\mathcal{S}^{\prime}$ is therefore

$$
\begin{aligned}
\mathcal{R}^{-1} \mathcal{S}^{\prime} \mathcal{R}= & 180_{0} 360_{231.43} 360_{102.86} 360_{334.29} 360_{205.71} 360_{77.14} 360_{308.57-} \\
& 360_{180} 360_{51.43} 360_{282.86} 360_{154.29} 360_{25.71} 360_{257.14-} \\
& 360_{128.57} 180_{0}
\end{aligned}
$$

This type of supercycle has almost the same effect as the combined phase inversion $/ 180^{\circ}$ pulse insertion supercycle discussed above. At the same time there is no need to apply very strong $\mathrm{rf}$ pulses. The timing complications associated with the inevitably finite duration of "strong" pulses are also avoided.

The cyclic permutation of an element creates a shift in the timing of the second sequence, disturbing the symmetry. In the case of $\gamma$-encoded pulse sequences, the timing shift may be compensated by modifying the rf phase of the second cycle, according to the phase-time relationship (equation (45)). This leads to the modified supercycle $[\mathcal{S}]_{0}\left[\mathcal{R}^{-1} \mathcal{S}^{\prime} \mathcal{R}\right]_{\delta}$, where the overall phase shift $\delta$ is given by

$$
\delta=-\frac{m}{\mu} \omega_{r} \tau_{\mathcal{R}}
$$


Here $\tau_{\mathcal{R}}$ is the duration of the cyclically permuted element $\mathcal{R}$, and $m / \mu$ is the ratio of space and spin quantum numbers selected by the $\gamma$-encoded $C N_{n}^{v}$ sequence.

In the case of equation (49), the symmetry $C 14_{4}^{5}$ selects the rotational components $\{l, m, \lambda, \mu\}=\{2, \pm 1,2, \mp 2\}$, and the permuted element has a duration $\tau_{\mathcal{P}}=2 \tau_{r} / 7$. The phase correction is therefore $\delta=\pi / 7=25.71^{\circ}$. The supercycle is

$$
\begin{aligned}
{[\mathcal{S}]_{0}\left[\mathcal{R}^{-1} \mathcal{S}^{\prime} \mathcal{R}\right]_{\delta}=} & 360_{0} 360_{128.57} 360_{257.14}- \\
& 360_{25.71} 360_{154.29} 360_{282.86} 360_{51.43} 360_{180}- \\
& 360_{308.57} 360_{77.14} 360_{205.71} 360_{334.29} 360_{102.86}- \\
& 360_{231.43} 180_{25.71} 360_{257.14} 360_{128.57} 360_{0}- \\
& 360_{231.43} 360_{102.86} 360_{334.29} 360_{205.71-} \\
& 360_{77.14} 360_{308.57} 360_{180} 360_{51.43} 360_{282.86}- \\
& 360_{154.29} 180_{25.71}
\end{aligned}
$$

This comprises one half of the SC14 sequence. ${ }^{39}$

This type of supercycle has beneficial effects on the CSA compensation of recoupling sequences, without destroying the $\gamma$-encoding.

\subsubsection{Additional Supercycles}

It is possible to construct supercycles by using consecutive $C N_{n}^{v}$ sequences based upon different basic elements $\mathcal{C}$. For example, Rienstra et al. ${ }^{\mathbf{3 8}}$ concatenated a $C 7_{2}^{1}$ sequence based upon the basic cycle $\mathcal{C}_{a}=360_{0} 360_{180}$ with a second $C 7_{2}^{1}$ sequence based upon a different basic cycle $\mathcal{C}_{b}=$ $180_{0} 360_{180} 180_{0}$. Some higher-order error terms of the individual cycles cancel out in the mixed supercycle $C 7_{2}^{1}(a) C 7_{2}^{1}(b)$.

More complex supercycles may be created by superimposing the basic supercycle types, and by transposing, mixing, and permuting the elements. For example, the full SC14 supercycle $^{39}$ is

$$
\left[[\mathcal{S}]_{0}\left[\mathcal{R}^{-1} \mathcal{S}^{\prime} \mathcal{R}\right]_{\delta}\right]_{0}\left[[\mathcal{S}]_{0}\left[\mathcal{R}^{-1} \mathcal{S}^{\prime} \mathcal{R}\right]_{\delta}\right]_{180}
$$

where $\mathcal{S}$ is a $C 14_{4}^{5}$ sequence using the basic element $\mathcal{C}=360_{0}$, the element $\mathcal{R}=180_{0}$ is cyclically permuted, and $\delta=25.71^{\circ}$. The CMR7 sequence ${ }^{\mathbf{3 8}}$ employs a particularly complicated supercycle structure.

At the moment, an empirical approach to supercycle construction prevails, since a complete higher-order theory has not yet been developed.

Supercycling should be implemented with care. In many cases, the supercycles are unnecessary, and readily harm the pulse sequence performance if they are used incorrectly.

\subsection{Rf Imperfections}

In practice, all $\mathrm{rf}$ pulse sequences are subject to various types of instrumental imperfection. Some typical imperfections of practical importance are:

1. Spatial inhomogeneity in the amplitude of the rf field over the sample volume;

2. Spatial inhomogeneity in the direction of the $\mathrm{rf}$ field over the sample volume, which translates into a phase modulation of the rf field as the sample rotates, ${ }^{25,86,87}$
3. Transients in the rf field upon changes in the driving field amplitude or phase, caused by the limited frequency bandwidth of the probe, $\boldsymbol{8 5 , 8 8}^{\circ}$

4. Instability or droop in the rf field applied to the probe;

5. Errors in the values of rf phase shifts;

6. Phase instability of the rf field.

Many of these imperfections have been investigated rather thoroughly, and some fit well into the framework of the symmetry theory. In particular, rf amplitude inhomogeneity may be included by using a set of interaction frame Hamiltonian terms with the quantum numbers $l=0, m=0, \lambda^{\text {eff }}=Z_{g}$ and $\mu=\{-1,0,+1\}$. Here $\lambda^{\text {eff }}$ is the 'effective spin rank' of the rf inhomogeneity terms and $Z_{g}$ is any even integer (' $g$ ' stands for gerade, which means 'even' in German).

It is surprising that the 'effective spin rank' $\lambda^{\text {eff }}$ of the rf inhomogeneity terms is even, since the rf spin Hamiltonian actually contains rank 1 spin operators. This is because the rf inhomogeneity terms depend on the phase of the rf field, even before transformation into the interaction frame. As a result, these terms behave differently to the others. ${ }^{\mathbf{8 9}}$

The rf inhomogeneity terms may be inserted into the selection rules and space-spin selection diagrams in the usual way. For example, it is easy to see that the rf inhomogeneity term $\left\{l, m, \lambda^{\text {eff }}, \mu\right\}=\left\{0,0, Z_{g}, 0\right\}$ is symmetry-allowed under $C 7_{2}^{1}$ and $R 18_{1}^{7}$ sequences, while the terms $\left\{0,0, Z_{g}, \pm 1\right\}$ are symmetry-forbidden. In order to achieve first-order $\mathrm{rf}$ compensation, one must therefore set the scaling factors for the symmetry allowed $\left\{0,0, Z_{g}, 0\right\}$ terms to zero. This may be done by using amplitude-modulated basic elements $\mathcal{C}$ or $\mathcal{R}$. It follows that symmetries such as $C 7_{2}^{1}$ and $R 18_{1}^{7}$ have firstorder compensation for $\mathrm{rf}$ inhomogeneity if they are based on amplitude-modulated elements.

In practice, higher-order terms are very important in determining the robustness of a pulse sequence with respect to rf amplitude deviations. These terms may be analyzed by using the selection rules equations (31) and (32). In many cases, it is simpler to use crude geometric arguments as a guideline. ${ }^{39}$ For example, although the symmetry $C 7_{2}^{1}$ is compensated for $\mathrm{rf}$ amplitude errors to first order (in the case of amplitude-modulated basic elements), geometrical arguments show that the second-order rf error terms are large. ${ }^{39}$ As a result, $C 7_{2}^{1}$ sequences must usually be based on elements which have a built-in rf error compensation, such as the original element $\mathcal{C}=360_{0} 360_{180},{ }^{36}$ or the "POST" element $\mathcal{C}=90_{0} 360_{180} 270_{0} \cdot{ }^{37}$ In Ref. ${ }^{39}$ it is shown by geometrical arguments that the symmetry $C 14_{4}^{5}$ has a higher degree of intrinsic rf error compensation, and that it is therefore feasible to use this symmetry together with the uncompensated basic element $\mathcal{C}=360_{0}$. This provides a two-fold reduction in the rf field requirements for a given spinning frequency.

The effect of rf transients has also been analyzed. ${ }^{85}$ A combination of electronic simulations and experimental measurements has led to the development of a simple analytical model for the transient behavior of the rf field at the sample. This model was used for accurate spin dynamical simulations during symmetry-based sequences, including the transient probe response. The effect of transients is minor, at least in the cases examined so far.

There is one common spectrometer imperfection which is a cause for real concern. In many contexts, the $R N_{n}^{v}$ class of 
pulse sequences is extremely sensitive to the precise value of the rf phase shift. As described above, $R N_{n}^{v}$ sequences are composed of elements with the alternating phase values $\pm \pi v / N$. Even small deviations from this precise phase shift value may be disastrous. ${ }^{85}$ A phase shift accuracy of $\pm 0.1^{\circ}$ or better is necessary for many applications of $R N_{n}^{v}$ sequences. In particularly demanding applications, such as long-range distance measurements, the phase shift accuracy should be an order of magnitude better still. Such extreme accuracy stretches current rf hardware. At the moment, only direct digital synthesis (DDS) of the rf wave produces sufficiently reliable and accurate phase shifts. Unfortunately, not all commercial spectrometers are equipped with such devices.

The extreme phase-shift sensitivity of $R N_{n}^{v}$ sequences may be understood using equation (46). This equation shows that a complete $R N_{n}^{v}$ sequence rotates resonant spins about the $z$-axis through the angle $2 N \phi$. Since the value of the phase shift is ideally $\phi=\pi \nu / N$, the overall rotation angle generated by one $R N_{n}^{v}$ sequence is ideally $\Phi=2 \pi v$ radians. This is an integer multiple of $360^{\circ}$, and so has no effect on the spin system. If a set of $K$ consecutive $R N_{n}^{v}$ sequences are applied, the overall rotation is through an angle $\Phi=2 \pi \nu K$, which also has no effect. However, if the value of $\phi$ is in error by a small angle $\Delta$, then the overall rotation over $K$ cycles is in excess by the angle $2 N K \Delta$. For example, in the case of a $R 14_{2}^{6}$ sequence, a phase deviation of only $\Delta=0.1^{\circ}$ leads to an accumulating error of $2.8^{\circ}$ per complete $R N_{n}^{v}$ sequence. Since a typical short-range pulse sequence employs around five complete sequences, the accumulated error rotation is about $14^{\circ}$. This is enough to degrade the double-quantum filtering efficiency significantly, especially since the double-quantum coherences are twice as sensitive as single-quantum coherences to phase shift errors. In a long-range application where around 50 complete sequences are used, the $0.1^{\circ}$ phase shift error is disastrous.

$C N_{n}^{v}$ sequences are much less sensitive to phase shift errors, since there are no accumulating rotations in this case.

The extreme phase shift sensitivity of $R N_{n}^{v}$ sequences may be removed by using the supercycle $\mathcal{S S}^{\prime}$. However, this method is only applicable to non- $\gamma$-encoded sequences. Work is under way towards a more general solution of this serious practical problem.

\subsection{Selection of Sequences}

Although the symmetry theory provides a sound framework for the search for suitable pulse sequences, this framework still allows enormous freedom. In order to develop a pulse sequence for a specific task, one must:

- Identify the space-spin components $\{l, m, \lambda, \mu\}$ which must be selected, and those that must be suppressed;

- Identify the set of suitable $C N_{n}^{v}$ or $R N_{n}^{v}$ symmetries;

- Identify the desired range of MAS frequencies;

- Identify a suitable basic element $\left(\mathcal{C}\right.$ in the case of $C N_{n}^{v}$ symmetry, or $\mathcal{R}$ in the case of $R N_{n}^{v}$ symmetry);

- Identify a suitable supercycle, if required.

For each problem, there are usually a large number of feasible symmetries, and within each symmetry, there is an infinity of possible basic elements.
This great freedom is both a strength and a disadvantage: It is a strength because it allows one to design a sequence tailored to the specific requirements of the experiment; it is a disadvantage because there are too many possibilities for a complete search.

The first two steps are easy: The application itself defines the desired and undesired space-spin components, and it is a straightforward task to identify the set of appropriate symmetries. This may be done manually using space-spin selection diagrams, or by using a Mathematica program ${ }^{\mathbf{6 3}}$ developed for this purpose (available at $h t t p: / / w w w . s o t o n . a c . u k / \sim m h l$ ).

The next stage is to choose the symmetry class and the basic element. Here one must pay attention to the following factors:

- The scaling factor for a given basic element $\mathcal{C}$ (or $\mathcal{R}$ ) under the symmetry class $C N_{n}^{v}$ (or $R N_{n}^{v}$ ) should be reasonably high.

- The required rf field depends on the basic element and also the symmetry class (more specifically, the ratio of $N$ to $n$ ). If the pulse sequence is intended to be broadband, it is usually desirable that the nutation frequency under the rf field should be as large as possible without being impractically large (see the subject of heteronuclear decoupling, discussed below).

- A rough guide to the relative performance of different symmetries is available by counting the number of symmetryallowed higher-order terms, using the selection rules in equations (31) and (32).

- It is sometimes unnecessary for the pulse sequence symmetry to suppress all of the undesired rotational components. Undesired components may also be suppressed by using a basic element which provides a zero scaling factor for the undesired rotational components. Supercycling may also be used to suppress the undesired symmetry-allowed components. These strategies are used in the SPC $5^{40}$ and C-REDOR ${ }^{67}$ schemes.

Previous experience in designing composite pulses ${ }^{\mathbf{7 - 9 , 6 1 , 6 2}}$ has been useful for guiding the choice of $\mathcal{C}$ and $\mathcal{R}$ elements (see Composite Pulses, Volume 2). For example, the composite pulse $360_{0} 360_{180}$ is known to be a robust rf cycle with good rf amplitude compensation and reasonably broadband behavior with respect to isotropic chemical shifts. Its chemical shift performance may be enhanced considerably by cyclicallypermuting a $90_{0}$ pulse, leading to $\mathcal{C}=90_{0} 360_{180} 270_{0}$. This 'permutationally offset-stabilized' (POST) element is a stalwart of liquid-state broadband decoupling experiments, ${ }^{7-9}$ and was used in the design of the POST-C7 sequence. ${ }^{37}$ Similarly, the element $\mathcal{R}=90_{0} 270_{180}$ is known as an offset-compensated $180^{\circ}$ composite pulse, ${ }^{7-9,61,62}$ and often proves to be a good choice in the context of $R N_{n}^{v}$ sequences. ${ }^{\mathbf{4 1 , 6 0}}$

More recently, semi-automated computer searches have been used to identify suitable sequences. For example, Brinkmann ${ }^{60}$ developed a combinatorial approach in which a large number of combinations of feasible symmetries, basic elements, and supercycle schemes are tested numerically by simulation under a variety of test situations.

The future may see a move towards more sophisticated basic elements, employing smoothly modulated pulse shapes rather than rectangular pulses. A full flowering of this field may require further improvements in the speed and flexibility of the electronic hardware. 


\section{HETERONUCLEAR SEQUENCES}

In practice, most samples contain several nuclear spin species. The selection rules and design principles may be extended to the case of heteronuclear spin interactions.

\subsection{Heteronuclear Spin Interactions}

Suppose that the rotating sample contains two interacting spin species, denoted $I$ and $S$, which are subjected to resonant $\mathrm{rf}$ fields at the corresponding nuclear Larmor frequencies. The nuclear spin interactions may be classified in terms of their spherical ranks under spatial (molecular) rotations, rotations of the $I$-spin polarizations by rf fields, rotations of the $S$-spin polarizations by rf fields, and rotations of the external magnetic field. The corresponding classification scheme is shown in Table 3. As may be seen, all interactions, with the exception of the homonuclear $J$-couplings, have different rotational properties. The symmetry theory of homonuclear rotorsynchronized pulse sequences is extended to the heteronuclear case by employing two different sets of spin quantum numbers.

In the presence of $\mathrm{rf}$ fields on both spin species $I$ and $S$, the interaction frame Hamiltonian may be described as a superposition of many rotational components:

$$
\mathcal{H}^{\Lambda}=\sum_{m=-l}^{+l} \sum_{\mu_{I}=-\lambda_{I}}^{+\lambda_{I}} \sum_{\mu_{S}=-\lambda_{S}}^{+\lambda_{S}} \mathcal{H}_{l m \lambda_{I} \mu_{I} \lambda_{S} \mu_{S}}^{\Lambda}
$$

as listed in Table 4.

The fundamental design task in heteronuclear spin systems is to design pulse sequence symmetries which select a small

Table 3 Rotational signatures of interactions in heteronuclear systems

\begin{tabular}{lcccc}
\hline Interaction & $\begin{array}{c}\text { Space } \\
\text { rank, } l\end{array}$ & $\begin{array}{c}I \text {-Spin } \\
\text { rank, } \lambda_{I}\end{array}$ & $\begin{array}{c}S \text {-Spin } \\
\text { rank, } \lambda_{S}\end{array}$ & $\begin{array}{c}\text { Field } \\
\text { rank }\end{array}$ \\
\hline$I$-Spin isotropic shift & 0 & 1 & 0 & 1 \\
$I$-Spin CSA & 2 & 1 & 0 & 1 \\
$I$-Spin $J$-coupling & 0 & 0 & 0 & 0 \\
$I$-Spin DD-coupling & 2 & 2 & 0 & 0 \\
$S$-Spin isotropic shift & 0 & 0 & 1 & 1 \\
$S$-Spin CSA & 2 & 0 & 1 & 1 \\
$S$-Spin $J$-coupling & 0 & 0 & 0 & 0 \\
$S$-Spin DD-coupling & 2 & 0 & 2 & 0 \\
$I S$-coupling & 0 & 1 & 1 & 0 \\
$I S$ DD-coupling & 2 & 1 & 1 & 0 \\
\hline
\end{tabular}

number of combinations of these terms, while suppressing others.

\subsection{Strong Heteronuclear Decoupling}

A common method for heteronuclear decoupling at the same time as homonuclear recoupling involves the application of a $C N_{n}^{v}$ or $R N_{n}^{v}$ sequence on one channel while a strong resonant $\mathrm{rf}$ field is applied to the second channel. If the rf field is strong enough, this suppresses the heteronuclear spin interactions, so that the two spin species are decoupled from each other.

If the strong resonant irradiation is on the $I$-spin channel, the appropriate selection rules are:

Case of $C N_{n}^{\nu_{S}}$ sequences with $I$-spin decoupling:

$$
\overline{\mathcal{H}}_{l m \lambda_{I} \mu_{I} \lambda_{S} \mu_{S}}^{(1)}=0 \quad \text { if } \quad m n-\mu v_{S} \neq N Z \quad \text { or if } \lambda_{I}=1
$$

Case of $R N_{n}^{v_{S}}$ sequences with $I$-spin decoupling:

$$
\overline{\mathcal{H}}_{l m \lambda_{I} \mu_{I} \lambda_{S} \mu_{S}}^{(1)}=0 \quad \text { if } m n-\mu v_{S} \neq \frac{N}{2} Z_{\lambda_{S}} \quad \text { or if } \lambda_{I}=1
$$

Empirically, it is found that effective heteronuclear decoupling requires a relatively large ratio of the nutation frequencies of the spins under their corresponding rf fields, i.e., if the decoupling irradiation is applied to the $I$-spins, while a recoupling sequence is applied to the $S$-spins, the ratio of nutation frequencies should be at least $\left|\omega_{\text {nut }}^{I} / \omega_{\text {nut }}^{S}\right|>\sim 3 .^{\mathbf{3 8}}$

In the absence of $S$-spin irradiation, two-pulse phase modulation (TPPM) of the decoupler field usually enhances the quality of the decoupling. ${ }^{\mathbf{1 0}}$ This does not usually appear to be the case when an rf field is also applied to the $S$-spins. Under some circumstances, however, the decoupling quality may be improved by placing the $I$-spin rf field well off-resonance, so as to approach or satisfy the Lee-Goldburg condition. ${ }^{11}$ These effects are of great practical importance but are not fully understood.

Since many of the applications of the symmetry-based sequences are in isotopically-labelled organic solids, good heteronuclear decoupling is of great importance. The need for a very strong decoupler field is a strong constraint on the applications of these pulse sequences, since typical NMR probes cannot tolerate simultaneous resonant $\mathrm{rf}$ fields on two irradiation channels for very long. A typical practical limit corresponds to nutation frequencies of around $100 \mathrm{kHz}$

\begin{tabular}{|c|c|c|c|c|c|c|}
\hline Interaction & $\begin{array}{l}\text { Space } \\
\text { rank, } l\end{array}$ & $\begin{array}{c}\text { Space } \\
\text { components, } m\end{array}$ & $\begin{array}{c}I-\text { Spin } \\
\operatorname{rank}, \lambda_{I}\end{array}$ & $\begin{array}{c}I \text {-Spin } \\
\text { components, } \mu_{I}\end{array}$ & $\begin{array}{l}S \text {-Spin } \\
\operatorname{rank}, \lambda_{S}\end{array}$ & $\begin{array}{c}S \text {-Spin } \\
\text { components, } \mu_{S}\end{array}$ \\
\hline$I$-Spin isotropic shift & 0 & 0 & 1 & $\{-1,0,1\}$ & 0 & 0 \\
\hline$I$-Spin CSA & 2 & $\{-2,-1,1,2\}$ & 1 & $\{-1,0,1\}$ & 0 & 0 \\
\hline$I$-Spin $J$-coupling & 0 & 0 & 0 & 0 & 0 & 0 \\
\hline I-Spin DD-coupling & 2 & $\{-2,-1,1,2\}$ & 2 & $\{-2,-1,0,1,2\}$ & 0 & 0 \\
\hline$S$-Spin isotropic shift & 0 & 0 & 0 & 0 & 1 & $\{-1,0,1\}$ \\
\hline$S$-Spin CSA & 2 & $\{-2,-1,1,2\}$ & 0 & 0 & 1 & $\{-1,0,1\}$ \\
\hline$S$-Spin $J$-coupling & 0 & 0 & 0 & 0 & 0 & 0 \\
\hline$S$-Spin DD-coupling & 2 & $\{-2,-1,1,2\}$ & 0 & 0 & 2 & $\{-2,-1,0,1,2\}$ \\
\hline IS J-coupling & 0 & 0 & 1 & $\{-1,0,1\}$ & 1 & $\{-1,0,1\}$ \\
\hline IS DD-coupling & 2 & $\{-2,-1,1,2\}$ & 1 & $\{-1,0,1\}$ & 1 & $\{-1,0,1\}$ \\
\hline
\end{tabular}

Table 4 Components of $I$ and $S$-spin interactions in the interaction frame of two resonant rf fields, in the case of exact magic-angle spinning 
for the $I$-spin channel and around $50 \mathrm{kHz}$ for the $S$-spin channel, applied for intervals of up to around $20 \mathrm{~ms}$. Since the symmetry-based sequences require a fixed ratio of the nutation frequency to the spinning frequency, the limitation in the tolerable $\mathrm{rf}$ power restricts the operational spinning frequency. For example, the $\mathrm{C} 7$ pulse sequence is typically limited to spinning frequencies of $7 \mathrm{kHz}$ or less. ${ }^{\mathbf{3 6}}$

\subsection{Single-Channel Sequences}

If the rf field is only applied to one spin species, the opportunities for symmetry selection are restricted. For example, if rf fields are applied at the $I$-spin Larmor frequency, but not at the $S$-spin Larmor frequency, the $S$-spin quantum number is restricted to $\mu_{S}=0$. In effect, only the first 5 columns in Table 4 are relevant. Under these circumstances, the rotational properties of the $I$-spin CSA and that of the $I S$ DD coupling are indistinguishable: Both have $l=2$ and $\lambda_{I}=1$. Similarly, the $I$-spin isotropic shift and that of the $I S J$-coupling both have $l=0$ and $\lambda_{I}=1$.

The selection rules for symmetry-based pulse sequences with rf irradiation on the $I$-spin channel are:

Case of $C N_{n}^{v_{I}}$ sequences with no $S$-spin rf fields:

$$
\overline{\mathcal{H}}_{l m \lambda_{I} \mu_{I} \lambda_{S} \mu_{S}}^{(1)}=0 \quad \text { if } m n-\mu v_{I} \neq N Z
$$

Case of $R N_{n}^{\nu_{I}}$ sequences with no $S$-spin rf fields:

$$
\overline{\mathcal{H}}_{l m \lambda_{I} \mu_{I} \lambda_{S} \mu_{S}}^{(1)}=0 \quad \text { if } m n-\mu v_{I} \neq \frac{N}{2} Z_{\lambda_{I}}
$$

Here $Z_{\lambda_{I}}$ is any integer with the same parity as the spin rank $\lambda_{I}$. These are the same as equations (27) and (28). The $S$-spin quantum numbers $\lambda_{S}$ and $\mu_{S}$ are irrelevant.

It follows that if the rf irradiation is restricted to one channel, the same pulse sequences recouple the heteronuclear DD interactions and the CSA interactions of the resonant spins.

\subsection{Dual Pulse Sequences}

If rf fields are applied at the Larmor frequencies of both spin species, there are many more opportunities for symmetry selection. As described in Ref. ${ }^{\mathbf{6 0}}$ four different symmetry classes may be envisaged, denoted $C N_{n}^{\nu_{I}, \nu_{S}}, C R N_{n}^{\nu_{I}, \nu_{S}}, R C N_{n}^{\nu_{I}, \nu_{S}}$ and $R N_{n}^{v_{I}, v_{S}}$ Figure 8. The basic elements may be different on the two channels, although they must have the same duration $n \tau_{r} / N$. The phase shift scheme for the $I$-spin irradiation follows the usual construction rule for $\mathrm{C}$-sequences (in the case of $C N_{n}^{v_{I}, v_{S}}$ or $C R N_{n}^{v_{I}, v_{S}}$ ), or for R-sequences (in the case of $R C N_{n}^{v_{I}, v_{S}}$ or $R N_{n}^{v_{I}, v_{S}}$ ), and depends on the symmetry numbers $N, n$ and $v_{I}$. Similarly, the phase shift scheme for the $S$-spin irradiation follows the construction rule for $\mathrm{C}$-sequences (in the case of $C N_{n}^{v_{I}, v_{S}}$ or $R C N_{n}^{v_{I}, v_{S}}$ ), or for R-sequences (in the case of $C R N_{n}^{v_{l}, v_{S}}$ or $R N_{n}^{v_{I}, v_{S}}$ ), and depends on the symmetry numbers $N, n$ and $v_{S}$.

The selection rules for dual sequences may be derived by extension of the single-channel case, and are given by the following expressions: ${ }^{60}$

Case of $C N_{n}^{v_{I}, v_{S}}$ sequences:

$$
\overline{\mathcal{H}}_{l m \lambda_{I} \mu_{I} \lambda_{S} \mu_{S}}^{(1)}=0 \quad \text { if } m n-\mu_{I} v_{I}-\mu_{S} v_{S} \neq N Z
$$

(a)

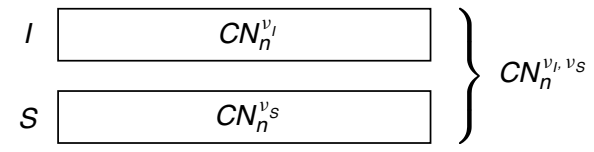

(b)

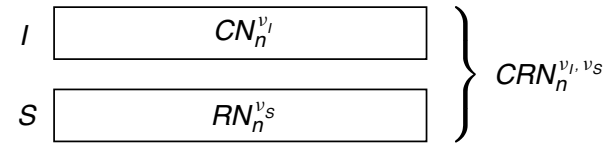

(c)

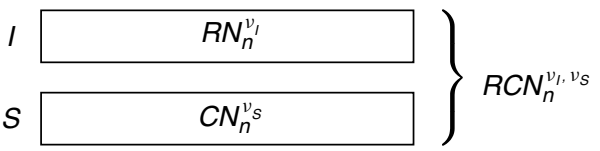

(d)

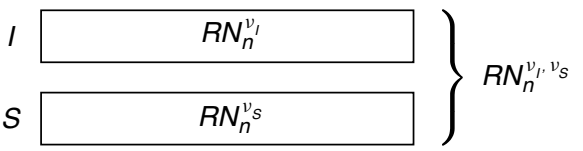

Figure 8 Naming convention for 2-channel heteronuclear pulse sequences

Case of $R C N_{n}^{v_{I}, v_{S}}$ sequences:

$$
\overline{\mathcal{H}}_{l m \lambda_{I} \mu_{I} \lambda_{S} \mu_{S}}^{(1)}=0 \quad \text { if } m n-\mu_{I} v_{I}-\mu_{S} v_{S} \neq \frac{N}{2} Z_{\lambda_{I}}
$$

Case of $C R N_{n}^{v_{I}, v_{S}}$ sequences:

$$
\overline{\mathcal{H}}_{l m \lambda_{I} \mu_{I} \lambda_{S} \mu_{S}}^{(1)}=0 \quad \text { if } m n-\mu_{I} v_{I}-\mu_{S} v_{S} \neq \frac{N}{2} Z_{\lambda_{S}}
$$

Case of $R N_{n}^{v_{l}, v_{S}}$ sequences:

$$
\overline{\mathcal{H}}_{l m \lambda_{I} \mu_{I} \lambda_{S} \mu_{S}}^{(1)}=0 \quad \text { if } m n-\mu_{I} v_{I}-\mu_{S} v_{S} \neq \frac{N}{2} Z_{\lambda_{I}+\lambda_{S}}
$$

The consequences of these selection rules may be explored by extended space-spin selection diagrams, as described in Ref. ${ }^{60}$ Expressions for the higher-order selection rules, and the dual-channel scaling factors, are also available. ${ }^{\mathbf{6 0}}$

The dual-channel selection rules enable the design of pulse sequences which select interactions on the basis of their full rotational properties, as detailed in Table 4. For example, the symmetry $C R 14_{3}^{2,2}$ suppresses all average Hamiltonian terms except those with the quantum numbers $\left\{l, m, \lambda_{I}, \mu_{I}, \lambda_{S}, \mu_{S}\right\}=\{0,0,0,0,0,0\}$ and $\{2, \mp 1,1, \pm 1,1$, $\pm 1\}$. This symmetry therefore enables $\gamma$-encoded doublequantum heteronuclear recoupling, with suppression of all homonuclear DD couplings, all chemical shift terms, and all heteronuclear $J$-couplings. ${ }^{\mathbf{6 0}}$

In principle, the selection rules (equations (58)-(61)) permit the rational design of homonuclear recoupling sequences with simultaneous heteronuclear decoupling. In principle, this approach could avoid the severe power requirements of the strong heteronuclear decoupling method (see above). However, no dual sequences with significant advantages over conventional decoupling methods have been found yet.

\section{APPLICATIONS}

This section presents a brief summary of some of the published applications of the symmetry theory sketched above. 
Sets of symmetry solutions appropriate for a number of different recoupling and decoupling problems are provided. The field is developing rapidly and these descriptions are by no means exhaustive.

\subsection{Double-Quantum Homonuclear Recoupling}

Double-quantum homonuclear recoupling has a number of important practical applications, including (see Homonuclear Recoupling Schemes in MAS NMR, Volume 4 and Solid State Investigations of Less-Common Nuclei with Small Magnetic Moments):

- Passing signals through double-quantum coherences allows the suppression of signals from isolated spins-1/2. This is particularly useful, for example, in the spectroscopy of multiply- ${ }^{13} \mathrm{C}$-labelled macromolecules, since the 'background' signals from naturally-occurring isolated ${ }^{13} \mathrm{C}$ nuclei are suppressed, allowing the selective observation of the labelled spin clusters. ${ }^{\mathbf{3 1}, 36,43,44}$

- Double-quantum spectroscopy, and double-quantum filtered correlation spectroscopy, are useful for peak assignments.

- Double-quantum coherences are sensitive to the correlations between local magnetic fields. Double-quantum NMR allows the relative orientations of different spin interactions to be determined, and hence the estimation of interbond angles and torsional angles. $\mathbf{4 3 , 4 4 , 7 6 - 8 1 , 9 0 , 9 1}$

- Double-quantum recoupling provides a route to the excitation of higher coherence orders..$^{81,92-94}$

Double-quantum homonuclear decoupling sequences may be classified according to whether or not they provide a $\gamma$ encoded recoupled Hamiltonian.

\subsection{1 $\gamma$-Encoded Double-Quantum Recoupling}

Many $C N_{n}^{v}$ symmetries provide $\gamma$-encoded double-quantum recoupling. As described above, $\gamma$-encoding simplifies the orientation-dependence of the recoupling, improving the clarity of the dipolar oscillations and the efficiency of the double-quantum excitation, and improving the time resolution.

Some of the symmetries achieving $\gamma$-encoded doublequantum recoupling selectively recouple the $m= \pm 1$ terms (Table 5), while others recouple the $m= \pm 2$ terms (Table 6). In general, the former are more efficient. The isotropic chemical shift terms are symmetry-allowed so the scaling factors of these terms must be set to zero by a suitable choice of the basic element $\mathcal{C}$. Table 5 includes the symmetry $C 7_{2}^{1}$, which was initially demonstrated with the basic element $\mathcal{C}=360_{0} 360_{180} \cdot{ }^{36}$ The 'POST' element $\mathcal{C}=90_{0} 360_{180} 270_{0}$ is more robust with respect to chemical shift offsets and is now favored. ${ }^{37}$ The symmetry $C 14_{4}^{5}$ allows operation at high spinning frequencies, if it is stabilized by the use of supercycles. ${ }^{39} \mathrm{~A}$ variant of $C 7_{2}^{1}$ employing a complex supercycle structure has been described. ${ }^{38}$ Sequences with the symmetry $C 5_{2}^{1}$ have also been used, in conjunction with a two-step supercycle to remove the components $\mu= \pm 1 .{ }^{40}$

The $C 7_{2}^{1}$ sequences have already been applied to 'real' systems, such as membrane proteins. For example, $C 7_{2}^{1}$ sequences were applied to a ${ }^{13} \mathrm{C}_{2}$-labelled sample of the photoreceptor protein bovine rhodopsin, allowing selective observation of the labelled sites with suppression of the background signals
Table 5 A set of $C N_{n}^{v}$ symmetries for $\gamma$-encoded homonuclear double-quantum recoupling, with selection of $\{l, m, \lambda, \mu\}=\{2, \pm 1,2$, $\pm 2\}$ or $\{2, \pm 1,2, \mp 2\}$ terms, and suppression of all CSA terms. The isotropic shift term $\{0,0,1,0\}$ and the homonuclear $J$-coupling $\{0,0,0,0\}$ are also symmetry-allowed. All inequivalent solutions in the range $N \leq 20, n \leq 10$ and $v \leq 10$ are shown

\begin{tabular}{llllll}
\hline$C 7_{1}^{3}$ & $C 9_{1}^{4}$ & $C 11_{1}^{5}$ & $C 13_{1}^{6}$ & $C 15_{1}^{7}$ & $C 17_{1}^{8}$ \\
$C 19_{1}^{9}$ & $C 7_{2}^{1}$ & $C 8_{2}^{1}$ & $C 8_{2}^{3}$ & $C 9_{2}^{1}$ & $C 10_{2}^{1}$ \\
$C 11_{2}^{1}$ & $C 12_{2}^{1}$ & $C 12_{2}^{5}$ & $C 13_{2}^{1}$ & $C 14_{2}^{1}$ & $C 15_{2}^{1}$ \\
$C 16_{2}^{1}$ & $C 16_{2}^{7}$ & $C 17_{2}^{1}$ & $C 18_{2}^{1}$ & $C 19_{2}^{1}$ & $C 20_{2}^{1}$ \\
$C 20_{2}^{9}$ & $C 7_{3}^{2}$ & $C 11_{3}^{4}$ & $C 13_{3}^{5}$ & $C 17_{3}^{7}$ & $C 19_{3}^{8}$ \\
$C 7_{4}^{2}$ & $C 9_{4}^{2}$ & $C 10_{4}^{3}$ & $C 11_{4}^{2}$ & $C 13_{4}^{2}$ & $C 14_{4}^{5}$ \\
$C 15_{4}^{2}$ & $C 17_{4}^{2}$ & $C 18_{4}^{7}$ & $C 19_{4}^{2}$ & $C 7_{5}^{1}$ & $C 9_{5}^{2}$ \\
$C 11_{5}^{3}$ & $C 13_{5}^{4}$ & $C 17_{5}^{6}$ & $C 19_{5}^{7}$ & $C 7_{6}^{3}$ & $C 8_{6}^{1}$ \\
$C 8_{6}^{3}$ & $C 10_{6}^{3}$ & $C 11_{6}^{3}$ & $C 13_{6}^{3}$ & $C 14_{6}^{3}$ & $C 16_{6}^{3}$ \\
$C 16_{6}^{5}$ & $C 17_{6}^{3}$ & $C 19_{6}^{3}$ & $C 20_{6}^{3}$ & $C 20_{6}^{7}$ & $C 9_{7}^{1}$ \\
$C 11_{7}^{2}$ & $C 13_{7}^{3}$ & $C 15_{7}^{4}$ & $C 17_{7}^{5}$ & $C 19_{7}^{6}$ & $C 7_{8}^{3}$ \\
$C 9_{8}^{4}$ & $C 10_{8}^{1}$ & $C 11_{8}^{4}$ & $C 13_{8}^{4}$ & $C 14_{8}^{3}$ & $C 15_{8}^{4}$ \\
$C 17_{8}^{4}$ & $C 18_{8}^{5}$ & $C 19_{8}^{4}$ & $C 7_{9}^{1}$ & $C 11_{9}^{1}$ & $C 13_{9}^{2}$ \\
$C 17_{9}^{4}$ & $C 19_{9}^{5}$ & $C 7_{10}^{2}$ & $C 8_{10}^{1}$ & $C 8_{10}^{3}$ & $C 9_{10}^{4}$ \\
$C 11_{10}^{5}$ & $C 12_{10}^{1}$ & $C 12_{10}^{5}$ & $C 13_{10}^{5}$ & $C 14_{10}^{5}$ & $C 16_{10}^{3}$ \\
$C 16_{10}^{5}$ & $C 17_{10}^{5}$ & $C 18_{10}^{5}$ & $C 19_{10}^{5}$ & & \\
\hline
\end{tabular}

Table 6 A set of $C N_{n}^{\nu}$ symmetries for $\gamma$-encoded homonuclear double-quantum recoupling, with selection of $\{l, m, \lambda, \mu\}=$ $\{2, \pm 2,2, \pm 2\}$ or $\{2, \pm 2,2, \mp 2\}$ terms, and suppression of all CSA terms. The isotropic shift term $\{0,0,1,0\}$ and the homonuclear $J$-coupling $\{0,0,0,0\}$ are also symmetry-allowed. All inequivalent solutions in the range $N \leq 20, n \leq 10$ and $v \leq 10$ are shown

\begin{tabular}{llllll}
\hline$C 8_{1}^{3}$ & $C 10_{1}^{4}$ & $C 12_{1}^{5}$ & $C 14_{1}^{6}$ & $C 16_{1}^{7}$ & $C 18_{1}^{8}$ \\
$C 20_{1}^{9}$ & $C 10_{2}^{3}$ & $C 14_{2}^{5}$ & $C 18_{2}^{7}$ & $C 8_{3}^{1}$ & $C 10_{3}^{2}$ \\
$C 14_{3}^{4}$ & $C 16_{3}^{5}$ & $C 20_{3}^{7}$ & $C 10_{4}^{1}$ & $C 14_{4}^{3}$ & $C 18_{4}^{5}$ \\
$C 8_{5}^{1}$ & $C 12_{5}^{1}$ & $C 14_{5}^{2}$ & $C 16_{5}^{3}$ & $C 18_{5}^{4}$ & $C 10_{6}^{1}$ \\
$C 14_{6}^{1}$ & $C 8_{7}^{3}$ & $C 10_{7}^{2}$ & $C 12_{7}^{1}$ & $C 16_{7}^{1}$ & $C 18_{7}^{2}$ \\
$C 20_{7}^{3}$ & $C 10_{8}^{3}$ & $C 14_{8}^{1}$ & $C 18_{8}^{1}$ & $C 8_{9}^{3}$ & $C 10_{9}^{4}$ \\
$C 14_{9}^{2}$ & $C 16_{9}^{1}$ & $C 20_{9}^{1}$ & $C 14_{10}^{3}$ & $C 18_{10}^{1}$ & \\
\hline
\end{tabular}

(see Bacteriorhodopsin \& Rhodopsin, Volume 2). The evolution of the ${ }^{13} \mathrm{C}_{2}$ double-quantum coherences in the presence of local fields from the neighboring protons allowed determination of a $\mathrm{H}-\mathrm{C}-\mathrm{C}-\mathrm{H}$ torsional angle in the retinylidene chromophore. ${ }^{43}$ A conformational change could be detected in a trapped photointermediate. ${ }^{44}$ The same experiment has also been demonstrated on carbohydrates. ${ }^{77,78}$ The experiment may be extended to allow the determination of ${ }^{15} \mathrm{~N}-{ }^{13} \mathrm{C}-{ }^{13} \mathrm{C}-{ }^{15} \mathrm{~N}$ torsional angles in peptides and proteins. ${ }^{79-81}$

If double-quantum recoupling sequences are applied to single-quantum coherences in clusters of more than two spins, triple-quantum coherences are developed. ${ }^{\mathbf{9 2}}$ These triplequantum coherences may also be used for torsional angle investigations. ${ }^{81}$ The double-quantum recoupled spin dynamics in three-spin-1/2 clusters have been investigated by twodimensional spectroscopy. ${ }^{39}$ High orders of coherence have been excited by applying $C 7{ }_{2}^{1}$ sequences to abundant protons. ${ }^{\mathbf{9 3}}$ The relaxation of zero-quantum coherence has been studied by using a $C 7_{2}^{1}$ sequence to excite double-quantum coherence, 
followed by a coherence transfer into zero-quantum coherence using a selective pulse pair. ${ }^{\mathbf{8 3}}$

$C 7_{2}^{1}$ sequences have also been applied to glasses, ${ }^{95-98}$ zeolites, ${ }^{\mathbf{9 9}}$ inorganic phosphates, ${ }^{\mathbf{1 0 0 - 1 0 2}}$ proteins ${ }^{\mathbf{1 0 3}}$ and drug molecules. 104

Figure 9 shows a two-dimensional double-quantum ${ }^{13} \mathrm{C}$ spectrum of $\left[\mathrm{U}_{-}{ }^{13} \mathrm{C}\right]$-L-tyrosine, obtained using the SC14 pulse sequence at a spinning frequency of $20 \mathrm{kHz} .{ }^{39}$ The utility of such spectra for peak assignment is obvious.

There are also a large number of $R N_{n}^{v}$ symmetries suitable for $\gamma$-encoded double-quantum recoupling. A set of symmetries which selectively recouple the $m= \pm 1$ terms are listed in Table 7. All of these symmetries suppress the isotropic chemical shift terms, and many of them are particularly robust with respect to chemical shift anisotropies. The symmetries $R 14_{2}^{6}$ and $R 22_{4}^{9}$ with $\mathcal{R}=90_{180} 270_{0}$ have been mainly used so far. ${ }^{41,85}$ Measurement of the excitation dynamics of the doublequantum coherences allows the accurate estimation of ${ }^{13} \mathrm{C}-{ }^{13} \mathrm{C}$

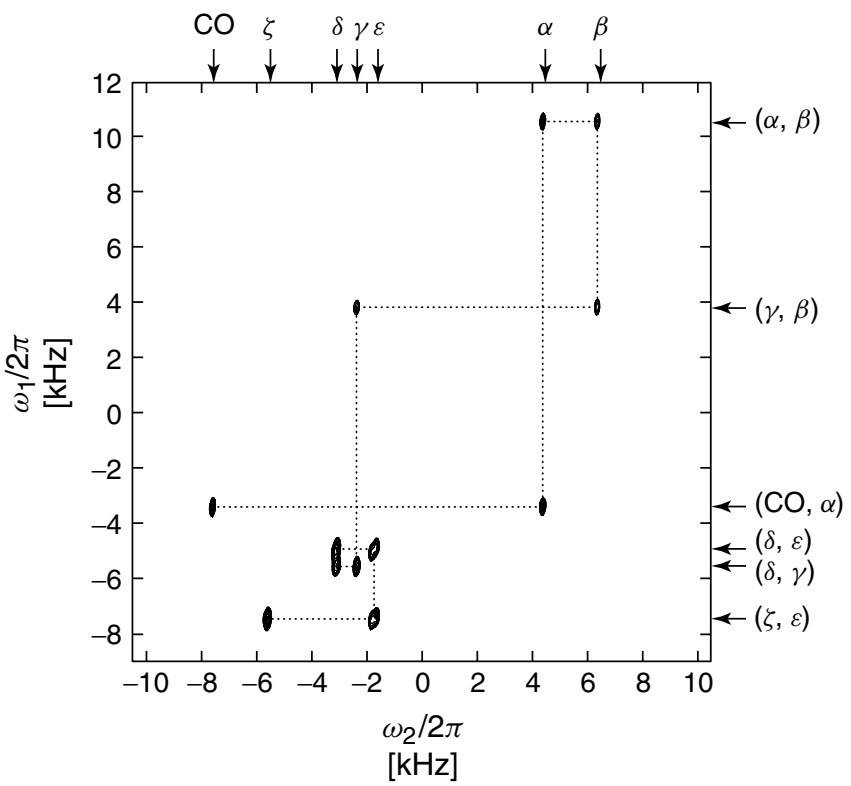

Figure 9 Two-dimensional double-quantum spectrum of $\left[\mathrm{U}-{ }^{13} \mathrm{C}\right]-$ L-tyrosine, obtained at a field of $9.4 \mathrm{~T}$ and a spinning frequency of $20 \mathrm{kHz}$, using the SC14 pulse sequence. The dashed lines are guides for the eye. (Adapted from Ref. ${ }^{\mathbf{3 9}}$ )

Table 7 A set of $R N_{n}^{v}$ symmetries for $\gamma$-encoded homonuclear double-quantum recoupling, with selection of $\{l, m, \lambda, \mu\}$ $=\{2, \pm 1,2, \pm 2\}$ or $\{2, \pm 1,2, \mp 2\}$ terms, and suppression of all isotropic shift and CSA terms. The homonuclear $J$-coupling $\{0,0,0,0\}$ is also symmetry-allowed. All inequivalent solutions in the range $N \leq 20, n, \leq 10$ and $v \leq 10$ are shown

\begin{tabular}{llll}
\hline$R 12_{2}^{1}$ & $R 12_{2}^{5}$ & $R 14_{2}^{1}$ & $R 14_{2}^{6}$ \\
$R 16_{2}^{1}$ & $R 16_{2}^{7}$ & $R 18_{2}^{1}$ & $R 18_{2}^{8}$ \\
$R 20_{2}^{1}$ & $R 20_{2}^{9}$ & $R 14_{4}^{2}$ & $R 14_{4}^{5}$ \\
$R 18_{4}^{2}$ & $R 18_{4}^{7}$ & & \\
\hline
\end{tabular}

bond lengths and medium-range distances in ${ }^{13} \mathrm{C}_{2}$-labelled systems, even in the presence of large CSA interactions. ${ }^{\mathbf{8 5}}$

As discussed above, the orientation-dependence of the recoupling under the $m= \pm 1$ sequences (Tables 5 and 7) is different from that under the $m= \pm 2$ sequences (Table 6). This is expected to improve the information content of the NMR spectroscopy of rotating oriented samples. ${ }^{\mathbf{7 5}}$ Preliminary results have been obtained. ${ }^{\mathbf{1 0 5}}$

\subsubsection{Non- $\gamma$-Encoded Double-Quantum Recoupling}

It is also possible to implement $2 \mathrm{Q}$ homonuclear recoupling without $\gamma$-encoding. An example is the back-to-back (BABA) recoupling sequence $\mathbf{1 0 2 , 1 0 6 - 1 0 8}^{\mathbf{1 0}}$ which is given by

$$
90_{0}-\tau-90_{180} 90_{90}-\tau-90_{270}
$$

The rf pulses are assumed to be strong and short and the delay $\tau$ is adjusted so that the complete sequence lasts one rotor period. This sequence corresponds to the first two elements of a $C 4_{2}^{1}$ sequence, using the basic element $\mathcal{C}=90_{0}-$ $\tau-90_{180}$. The $C 4_{2}^{1}$ symmetry implements double-quantum recoupling, but also allows many unwanted terms, such as the isotropic chemical shift term $\{l, m, \lambda, \mu\}=\{0,0,1,0\}$, CSA terms of the form $\{l, m, \lambda, \mu\}=\{2, \pm 2,1,0\}$, and zeroquantum DD terms of the form $\{l, m, \lambda, \mu\}=\{2, \pm 2,2,0\}$. However, the scaling factors for all of these terms vanish if the rf pulses are made infinitely short. In this limit, the only symmetry-allowed terms in the first-order average Hamiltonian are of the form $\{l, m, \lambda, \mu\}=\{2, \pm 1,2, \pm 2\}$ and $\{2, \pm 1,2, \mp 2\}$. In the limit of very strong rf fields, BABA therefore implements non- $\gamma$-encoded homonuclear DD recoupling with compensation for chemical shifts. Although the lack of $\gamma$-encoding has disadvantages, the same property allows the robustness of the sequence to be enhanced by constructing a $\mathcal{S S}^{\prime}$ supercycle, i.e.,

$$
\begin{aligned}
& 90_{0}-\tau-90_{180} 90_{90}-\tau-90_{270} 90_{0}-\tau-90_{180} \\
& 90_{270}-\tau-90_{90}
\end{aligned}
$$

In addition, the absence of $\gamma$-encoding causes orientationally-induced modulations in 2D experiments, which may be analyzed in order to estimate internuclear distances. ${ }^{\mathbf{6 , 6 9}}$ The

Table 8 A set of $C N_{n}^{v}$ symmetries for non- $\gamma$-encoded homonuclear double-quantum recoupling. The symmetries $C 6_{2}^{1}$ and $C 6_{8}^{1}$ suppress all CSA terms and all homonuclear DD terms except $\quad\{l, m, \lambda, \mu\}=\{2, \pm 2,2, \mp 2\}$ and $\{2, \pm 1,2, \pm 2\}$. The symmetries $C 6_{4}^{1}$ and $C 6_{10}^{1}$ suppress all CSA terms and all homonuclear DD terms except $\{l, m, \lambda, \mu\}=\{2, \pm 2,2, \pm 2\}$ and $\{2, \pm 1,2, \mp 2\}$. The isotropic shift term $\{0,0,1,0\}$ and the homonuclear $J$-coupling $\{0,0,0,0\}$ are symmetryallowed. All inequivalent solutions in the range $N \leq 20, n \leq 10$ and $v \leq 10$ are shown

\begin{tabular}{llll}
\hline$C 6_{2}^{1}$ & $C 6_{4}^{1}$ & $C 6_{8}^{1}$ & $C 6_{10}^{1}$ \\
\hline
\end{tabular}


supercycled version of BABA has been applied to a variety of systems including phosphate networks ${ }^{\mathbf{1 0 6}, 107}$ and glasses. ${ }^{\mathbf{1 0 8}}$

There are several $C$-symmetries which provide non- $\gamma$ encoded 2Q recoupling with suppression of all CSA terms, even in the case of finite rf fields (see Table 8). The isotropic chemical shift terms are readily suppressed by an appropriate choice of the basic element. These $\mathrm{C}$-sequences may provide an alternative to BABA in the case of limited rf field strength.

\subsection{Zero-Quantum Homonuclear Recoupling}

The radio-frequency-driven recoupling (RFDR) pulse sequence is widely used to transfer longitudinal magnetization between dipolar coupled spin sites in the solid state (see Homonuclear Recoupling Schemes in MAS NMR, Volume 4). The most common application is as the mixing interval in a two-dimensional experiment. If the $J$-couplings are neglected, the 2D spectrum displays cross peaks which indicate the spatial proximity of spin sites.

The most popular version of RFDR employs the so-called XY8 phase scheme. ${ }^{33,34}$ This corresponds to a sequence of strong $180^{\circ}$ pulses, spaced at intervals of one rotor period, with rf phases of $\{0,90,0,90,90,0,90,0\}$. This corresponds to a $\mathcal{S S}^{\prime}$ supercycle of a $R 4_{4}^{1}$ sequence, with an overall $45^{\circ}$ phase shift. This is indeed an appropriate symmetry for zeroquantum homonuclear recoupling, as may be seen from the list of solutions in Table 9. Symmetries such as $R 4_{4}^{1}$ select terms of the form $\{l, m, \lambda, \mu\}=\{2, \pm 1,2,0\}$ and $\{2, \pm 2,2,0\}$. The recoupling is not $\gamma$-encoded, which harms its efficiency in a
Table 9 A set of $R N_{n}^{v}$ symmetries for recoupling of the $\{l, m, \lambda, \mu\}=\{2, \pm 2,2,0\}$ and $\{2, \pm 1,2,0\}$ terms with suppression of all other homonuclear DD terms, CSA terms and isotropic shift terms. The homonuclear $J$-coupling $\{0,0,0,0\}$ is symmetry-allowed. All inequivalent solutions in the range $N \leq 40, n \leq 10$ and $v \leq 20$ are shown

\begin{tabular}{llll}
$R 4_{4}^{1}$ & $R 6_{6}^{1}$ & $R 6_{6}^{2}$ & $R 4_{8}^{1}$ \\
$R 8_{8}^{1}$ & $R 8_{8}^{3}$ & $R 10_{10}^{1}$ & $R 10_{10}^{2}$ \\
$R 10_{10}^{3}$ & $R 10_{10}^{4}$ & & \\
\hline
\end{tabular}

non-oriented sample, but the lack of $\gamma$-encoding permits the use of the stabilizing supercycle $\mathcal{S S}^{\prime}$.

The curious aspect of RFDR is that the scaling factor for the symmetry-allowed terms tends to zero in the limit of a strong rf field. Indeed, simulations show that the only reason RFDR works is because of the finite duration of practical rf pulses, interference from chemical shifts, ${ }^{\mathbf{3 4}}$ and the significant effect of $J$-couplings (which may even be dominant in some circumstances). Ishii ${ }^{64}$ has given a symmetrybased analysis of RFDR with finite rf pulses, and proposed the use of symmetries such as $R 4_{4}^{1}$ and $R 6_{6}^{1}$. Brinkmann and Schmedt auf der Günne ${ }^{\mathbf{6 5}}$ have demonstrated additional symmetries and explored the use of more complex $\mathcal{R}$ elements in order to allow operation at high spinning frequencies. One recommended sequence involves the supercycle $\left[\mathcal{S S}^{\prime}\right]_{0}\left[\mathcal{S S}^{\prime}\right]_{120}\left[\mathcal{S S}^{\prime}\right]_{240}$ where $\mathcal{S}$ is a $R 6_{6}^{2}$ sequence using the

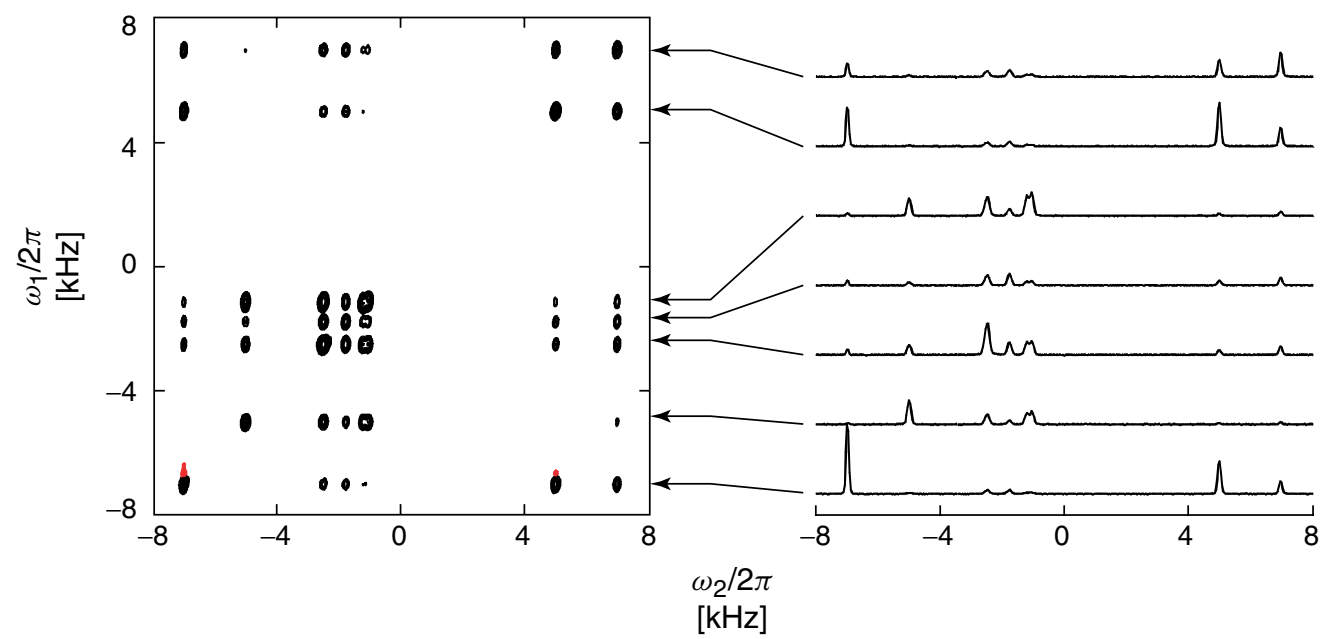

Figure 10 Two-dimensional correlation spectrum of $\left[\mathrm{U}-{ }^{13} \mathrm{C}\right]-\mathrm{L}-t y r o s i n e$, obtained at a field of $9.4 \mathrm{~T}$ and a spinning frequency of $23 \mathrm{kHz}$, using a supercycled $R 6_{6}^{2}$ pulse sequence, with a mixing interval of 9.9 ms. (Adapted from Ref. ${ }^{65}$ )

Table 10 A set of $R N_{n}^{v}$ symmetries for $\gamma$-encoded recoupling of the $\{l, m, \lambda, \mu\}=\{2, \pm 2,2, \pm 1\}$ or $\{2, \pm 2,2, \mp 1\}$ components of the homonuclear DD couplings with suppression of all other homonuclear DD terms, CSA terms and isotropic shift terms. The homonuclear $J$-coupling $\{0,0,0,0\}$ is symmetry-allowed. All inequivalent solutions in the range $N \leq 20, n \leq 10$ and $v \leq 10$ are shown

\begin{tabular}{llllllllll}
\hline$R 10_{1}^{2}$ & $R 12_{1}^{2}$ & $R 14_{1}^{2}$ & $R 16_{1}^{2}$ & $R 18_{1}^{2}$ & $R 20_{1}^{2}$ & $R 14_{2}^{4}$ & $R 18_{2}^{4}$ & $R 10_{3}^{4}$ & $R 14_{3}^{6}$ \\
$R 16_{3}^{6}$ & $R 20_{3}^{6}$ & $R 14_{4}^{8}$ & $R 18_{4}^{8}$ & $R 12_{5}^{10}$ & $R 14_{5}^{10}$ & $R 16_{5}^{10}$ & $R 18_{5}^{10}$ & $R 14_{6}^{12}$ & $R 20_{6}^{12}$ \\
$R 10_{7}^{4}$ & $R 12_{7}^{2}$ & $R 16_{7}^{14}$ & $R 18_{7}^{14}$ & $R 20_{7}^{14}$ & $R 14_{8}^{2}$ & $R 18_{8}^{16}$ & $R 10_{9}^{2}$ & $R 14_{9}^{4}$ & $R 16_{9}^{2}$ \\
$R 20_{9}^{18}$ & $R 14_{10}^{6}$ & $R 18_{10}^{2}$ & & & & & & & \\
\hline
\end{tabular}


Table 11 A set of $C N_{n}^{v}$ symmetries for selection of homonuclear $J$-couplings $\{l, m, \lambda, \mu\}=\{0,0,0,0\}$ with suppression of all homonuclear DD couplings and CSA terms. Some isotropic shift terms are also symmetry-allowed. All inequivalent solutions in the range $N \leq 20, n \leq 10$ and $v \leq 10$ are shown

\begin{tabular}{|c|c|c|c|c|c|c|c|c|c|}
\hline$C 3_{1}^{0}$ & $C 4_{1}^{0}$ & $C 5_{1}^{0}$ & $C 6_{1}^{0}$ & $C 6_{1}^{3}$ & $C 7_{1}^{0}$ & $C 8_{1}^{0}$ & $C 8_{1}^{4}$ & $C 9_{1}^{0}$ & $C 9_{1}^{3}$ \\
\hline$C 10_{1}^{0}$ & $C 10_{1}^{3}$ & $C 10_{1}^{5}$ & $C 11_{1}^{0}$ & $C 11_{1}^{3}$ & $C 11_{1}^{4}$ & $C 12_{1}^{0}$ & $C 12_{1}^{3}$ & $C 12_{1}^{4}$ & $C 12_{1}^{6}$ \\
\hline$C 13_{1}^{0}$ & $C 13_{1}^{3}$ & $C 13_{1}^{4}$ & $C 13_{1}^{5}$ & $C 14_{1}^{0}$ & $C 14_{1}^{3}$ & $C 14_{1}^{4}$ & $C 14_{1}^{5}$ & $C 14_{1}^{7}$ & $C 15_{1}^{0}$ \\
\hline$C 15_{1}^{3}$ & $C 15_{1}^{4}$ & $C 15_{1}^{5}$ & $C 15_{1}^{6}$ & $C 16_{1}^{0}$ & $C 16_{1}^{3}$ & $C 16_{1}^{4}$ & $C 16_{1}^{5}$ & $C 16_{1}^{6}$ & $C 16_{1}^{8}$ \\
\hline$C 17_{1}^{0}$ & $C 17_{1}^{3}$ & $C 17_{1}^{4}$ & $C 17_{1}^{5}$ & $C 17_{1}^{6}$ & $C 17_{1}^{7}$ & $C 18_{1}^{0}$ & $C 18_{1}^{3}$ & $C 18_{1}^{4}$ & $C 18_{1}^{5}$ \\
\hline$C 18_{1}^{6}$ & $C 18_{1}^{7}$ & $C 18_{1}^{9}$ & $C 19_{1}^{0}$ & $C 19_{1}^{3}$ & $C 19_{1}^{4}$ & $C 19_{1}^{5}$ & $C 19_{1}^{6}$ & $C 19_{1}^{7}$ & $C 19_{1}^{8}$ \\
\hline$C 20_{1}^{0}$ & $C 20_{1}^{3}$ & $C 20_{1}^{4}$ & $C 20_{1}^{5}$ & $C 20_{1}^{6}$ & $C 20_{1}^{7}$ & $C 20_{1}^{8}$ & $C 20_{1}^{10}$ & $C 3_{2}^{0}$ & $C 5_{2}^{0}$ \\
\hline$C 6_{2}^{3}$ & $C 7_{2}^{0}$ & $C 9_{2}^{0}$ & $C 9_{2}^{3}$ & $C 10_{2}^{5}$ & $C 11_{2}^{0}$ & $C 11_{2}^{3}$ & $C 11_{2}^{5}$ & $C 12_{2}^{3}$ & $C 13_{2}^{0}$ \\
\hline$C 13_{2}^{3}$ & $C 13_{2}^{5}$ & $C 13_{2}^{6}$ & $C 14_{2}^{3}$ & $\mathrm{C} 14_{2}^{7}$ & $C 15_{2}^{0}$ & $C 15_{2}^{3}$ & $C 15_{2}^{5}$ & $C 15_{2}^{6}$ & $C 15_{2}^{7}$ \\
\hline$C 16_{2}^{3}$ & $C 16_{2}^{5}$ & $C 17_{2}^{0}$ & $C 17_{2}^{3}$ & $C 17_{2}^{5}$ & $C 17_{2}^{6}$ & $C 17_{2}^{7}$ & $C 17_{2}^{8}$ & $C 18_{2}^{3}$ & $C 18_{2}^{5}$ \\
\hline$C 18_{2}^{9}$ & $C 19_{2}^{0}$ & $C 19_{2}^{3}$ & $C 19_{2}^{5}$ & $C 19_{2}^{6}$ & $C 19_{2}^{7}$ & $C 19_{2}^{8}$ & $C 19_{2}^{9}$ & $C 20_{2}^{3}$ & $C 20_{2}^{5}$ \\
\hline $\mathrm{C} 2 \mathrm{O}_{2}^{7}$ & $C 4_{3}^{0}$ & $C 5_{3}^{0}$ & $C 7_{3}^{0}$ & $C 8_{3}^{0}$ & $C 8_{3}^{4}$ & $C 9_{3}^{1}$ & $C 9_{3}^{2}$ & $C 9_{3}^{4}$ & $\mathrm{C} 10_{3}^{0}$ \\
\hline$C 10_{3}^{1}$ & $C 10_{3}^{5}$ & $C 11_{3}^{0}$ & $C 11_{3}^{1}$ & $C 11_{3}^{2}$ & $C 12_{3}^{1}$ & $C 12_{3}^{2}$ & $C 12_{3}^{4}$ & $C 12_{3}^{5}$ & $\mathrm{C} 13_{3}^{0}$ \\
\hline$C 13_{3}^{1}$ & $C 13_{3}^{2}$ & $C 13_{3}^{4}$ & $C 14_{3}^{0}$ & $\mathrm{C} 14_{3}^{1}$ & $C 14_{3}^{2}$ & $C 14_{3}^{5}$ & $C 14_{3}^{7}$ & $C 15_{3}^{1}$ & $C 15_{3}^{2}$ \\
\hline$C 15_{3}^{4}$ & $C 15_{3}^{5}$ & $C 15_{3}^{7}$ & $C 16_{3}^{0}$ & $C 16_{3}^{1}$ & $C 16_{3}^{2}$ & $C 16_{3}^{4}$ & $C 16_{3}^{7}$ & $C 16_{3}^{8}$ & $C 17_{3}^{0}$ \\
\hline$C 17_{3}^{1}$ & $C 17_{3}^{2}$ & $C 17_{3}^{4}$ & $C 17_{3}^{5}$ & $C 17_{3}^{8}$ & $C 18_{3}^{1}$ & $C 18_{3}^{2}$ & $C 18_{3}^{4}$ & $C 18_{3}^{5}$ & $C 18_{3}^{7}$ \\
\hline$C 18_{3}^{8}$ & $C 19_{3}^{0}$ & $C 19_{3}^{1}$ & $C 19_{3}^{2}$ & $C 19_{3}^{4}$ & $C 19_{3}^{5}$ & $C 19_{3}^{7}$ & $C 19_{3}^{9}$ & $C 20_{3}^{0}$ & $C 20_{3}^{1}$ \\
\hline $\mathrm{C} 2 \mathrm{O}_{3}^{2}$ & $C 20_{3}^{4}$ & $C 20_{3}^{5}$ & $C 20_{3}^{8}$ & $\mathrm{C} 2 \mathrm{O}_{3}^{9}$ & $C 20_{3}^{10}$ & $C 3_{4}^{0}$ & $C 5_{4}^{0}$ & $C 6_{4}^{3}$ & $C 7_{4}^{0}$ \\
\hline$C 9_{4}^{0}$ & $C 9_{4}^{3}$ & $C 10_{4}^{5}$ & $C 11_{4}^{0}$ & $C 11_{4}^{1}$ & $C 11_{4}^{5}$ & $C 12_{4}^{1}$ & $C 12_{4}^{3}$ & $C 12_{4}^{5}$ & $C 13_{4}^{0}$ \\
\hline$C 13_{4}^{1}$ & $C 13_{4}^{3}$ & $C 13_{4}^{6}$ & $C 14_{4}^{1}$ & $\mathrm{C} 14_{4}^{7}$ & $C 15_{4}^{0}$ & $C 15_{4}^{1}$ & $C 15_{4}^{3}$ & $C 15_{4}^{5}$ & $C 15_{4}^{6}$ \\
\hline$C 16_{4}^{1}$ & $C 16_{4}^{3}$ & $C 16_{4}^{5}$ & $C 16_{4}^{7}$ & $C 17_{4}^{0}$ & $C 17_{4}^{1}$ & $C 17_{4}^{3}$ & $C 17_{4}^{5}$ & $C 17_{4}^{6}$ & $C 17_{4}^{7}$ \\
\hline$C 18_{4}^{1}$ & $C 18_{4}^{3}$ & $C 18_{4}^{9}$ & $C 19_{4}^{0}$ & $C 19_{4}^{1}$ & $C 19_{4}^{3}$ & $C 19_{4}^{5}$ & $C 19_{4}^{6}$ & $C 19_{4}^{7}$ & $C 19_{4}^{9}$ \\
\hline$C 20_{4}^{1}$ & $C 20_{4}^{3}$ & $C 20_{4}^{5}$ & $\mathrm{C} 2 \mathrm{O}_{4}^{7}$ & $\mathrm{C} 2 \mathrm{O}_{4}^{9}$ & $C 3_{5}^{0}$ & $C 4_{5}^{0}$ & $C 6_{5}^{0}$ & $C 6_{5}^{3}$ & $C 7_{5}^{0}$ \\
\hline$C 8_{5}^{0}$ & $C 8_{5}^{4}$ & $C 9_{5}^{0}$ & $C 9_{5}^{3}$ & $C 11_{5}^{0}$ & $C 11_{5}^{2}$ & $C 11_{5}^{4}$ & $C 12_{5}^{0}$ & $C 12_{5}^{3}$ & $C 12_{5}^{4}$ \\
\hline$C 12_{5}^{6}$ & $C 13_{5}^{0}$ & $C 13_{5}^{1}$ & $C 13_{5}^{2}$ & $C 13_{5}^{6}$ & $C 14_{5}^{0}$ & $C 14_{5}^{1}$ & $C 14_{5}^{3}$ & $C 14_{5}^{6}$ & $C 14_{5}^{7}$ \\
\hline$C 15_{5}^{1}$ & $C 15_{5}^{2}$ & $C 15_{5}^{3}$ & $C 15_{5}^{4}$ & $C 15_{5}^{6}$ & $C 15_{5}^{7}$ & $C 16_{5}^{0}$ & $C 16_{5}^{1}$ & $C 16_{5}^{2}$ & $C 16_{5}^{4}$ \\
\hline$C 16_{5}^{7}$ & $C 16_{5}^{8}$ & $C 17_{5}^{0}$ & $C 17_{5}^{1}$ & $C 17_{5}^{2}$ & $C 17_{5}^{3}$ & $C 17_{5}^{4}$ & $C 17_{5}^{8}$ & $C 18_{5}^{0}$ & $C 18_{5}^{1}$ \\
\hline$C 18_{5}^{2}$ & $C 18_{5}^{3}$ & $C 18_{5}^{6}$ & $C 18_{5}^{7}$ & $C 18_{5}^{9}$ & $C 19_{5}^{0}$ & $C 19_{5}^{1}$ & $C 19_{5}^{2}$ & $C 19_{5}^{3}$ & $C 19_{5}^{4}$ \\
\hline$C 19_{5}^{6}$ & $C 19_{5}^{8}$ & $C 20_{5}^{1}$ & $C 20_{5}^{2}$ & $C 20_{5}^{3}$ & $C 20_{5}^{4}$ & $C 20_{5}^{6}$ & $C 20_{5}^{7}$ & $C 20_{5}^{8}$ & $C 20_{5}^{9}$ \\
\hline$C 5_{6}^{0}$ & $C 7_{6}^{0}$ & $C 9_{6}^{1}$ & $C 9_{6}^{2}$ & $C 9_{6}^{4}$ & $C 10_{6}^{5}$ & $C 11_{6}^{0}$ & $C 11_{6}^{2}$ & $C 11_{6}^{4}$ & $C 13_{6}^{0}$ \\
\hline$C 13_{6}^{2}$ & $C 13_{6}^{4}$ & $C 13_{6}^{5}$ & $C 14_{6}^{5}$ & $C 14_{6}^{7}$ & $C 15_{6}^{1}$ & $C 15_{6}^{2}$ & $C 15_{6}^{4}$ & $C 15_{6}^{5}$ & $C 15_{6}^{7}$ \\
\hline$C 16_{6}^{1}$ & $C 16_{6}^{7}$ & $C 17_{6}^{0}$ & $C 17_{6}^{1}$ & $C 17_{6}^{2}$ & $C 17_{6}^{4}$ & $C 17_{6}^{7}$ & $C 17_{6}^{8}$ & $C 18_{6}^{1}$ & $C 18_{6}^{5}$ \\
\hline$C 18_{6}^{7}$ & $C 19_{6}^{0}$ & $C 19_{6}^{1}$ & $C 19_{6}^{2}$ & $C 19_{6}^{4}$ & $C 19_{6}^{5}$ & $C 19_{6}^{8}$ & $C 19_{6}^{9}$ & $C 20_{6}^{1}$ & $C 20_{6}^{5}$ \\
\hline$C 20_{6}^{9}$ & $C 3_{7}^{0}$ & $C 4_{7}^{0}$ & $C 5_{7}^{0}$ & $C 6_{7}^{0}$ & $C 6_{7}^{3}$ & $C 8_{7}^{0}$ & $C 8_{7}^{4}$ & $C 9_{7}^{0}$ & $C 9_{7}^{3}$ \\
\hline$C 10_{7}^{0}$ & $C 10_{7}^{1}$ & $C 10_{7}^{5}$ & $C 11_{7}^{0}$ & $C 11_{7}^{1}$ & $C 11_{7}^{5}$ & $C 12_{7}^{0}$ & $C 12_{7}^{3}$ & $C 12_{7}^{4}$ & $C 12_{7}^{6}$ \\
\hline$C 13_{7}^{0}$ & $C 13_{7}^{2}$ & $C 13_{7}^{4}$ & $C 13_{7}^{5}$ & $C 15_{7}^{0}$ & $C 15_{7}^{2}$ & $C 15_{7}^{3}$ & $C 15_{7}^{5}$ & $C 15_{7}^{6}$ & $C 16_{7}^{0}$ \\
\hline$C 16_{7}^{3}$ & $C 16_{7}^{4}$ & $C 16_{7}^{5}$ & $C 16_{7}^{6}$ & $C 16_{7}^{8}$ & $C 17_{7}^{0}$ & $C 17_{7}^{1}$ & $C 17_{7}^{2}$ & $C 17_{7}^{4}$ & $C 17_{7}^{6}$ \\
\hline$C 17_{7}^{8}$ & $C 18_{7}^{0}$ & $C 18_{7}^{1}$ & $C 18_{7}^{3}$ & $C 18_{7}^{5}$ & $C 18_{7}^{6}$ & $C 18_{7}^{8}$ & $C 18_{7}^{9}$ & $C 19_{7}^{0}$ & $C 19_{7}^{1}$ \\
\hline$C 19_{7}^{2}$ & $C 19_{7}^{3}$ & $C 19_{7}^{4}$ & $C 19_{7}^{8}$ & $C 19_{7}^{9}$ & $C 20_{7}^{0}$ & $C 20_{7}^{1}$ & $C 20_{7}^{2}$ & $C 20_{7}^{4}$ & $C 20_{7}^{5}$ \\
\hline$C 20_{7}^{8}$ & $C 20_{7}^{9}$ & $C 20_{7}^{10}$ & $C 3_{8}^{0}$ & $C 5_{8}^{0}$ & $C 6_{8}^{3}$ & $C 7_{8}^{0}$ & $C 9_{8}^{0}$ & $C 9_{8}^{3}$ & $C 10_{8}^{5}$ \\
\hline$C 11_{8}^{0}$ & $C 11_{8}^{1}$ & $C 11_{8}^{2}$ & $C 12_{8}^{1}$ & $C 12_{8}^{3}$ & $C 12_{8}^{5}$ & $C 13_{8}^{0}$ & $C 13_{8}^{1}$ & $C 13_{8}^{2}$ & $C 13_{8}^{6}$ \\
\hline$C 14_{8}^{5}$ & $C 14_{8}^{7}$ & $C 15_{8}^{0}$ & $C 15_{8}^{2}$ & $C 15_{8}^{3}$ & $C 15_{8}^{5}$ & $C 15_{8}^{6}$ & $C 17_{8}^{0}$ & $C 17_{8}^{2}$ & $C 17_{8}^{3}$ \\
\hline$C 17_{8}^{5}$ & $C 17_{8}^{6}$ & $C 17_{8}^{7}$ & $C 18_{8}^{3}$ & $\mathrm{C} 18_{8}^{7}$ & $C 18_{8}^{9}$ & $C 19_{8}^{\circ}$ & $C 19_{8}^{1}$ & $C 19_{8}^{2}$ & $C 19^{\circ}$ \\
\hline$C 19_{8}^{6}$ & $\mathrm{C} 19_{8}^{7}$ & $C 19_{8}^{9}$ & $C 20_{8}^{1}$ & $\mathrm{C} 2 \mathrm{O}_{8}^{3}$ & $C 20_{8}^{5}$ & $\mathrm{C} 2 \mathrm{O}_{8}^{7}$ & $C 20_{8}^{9}$ & $C 4_{9}^{0}$ & $C 5_{9}^{0}$ \\
\hline$C 7_{9}^{0}$ & $C 8_{9}^{0}$ & $C 8_{9}^{4}$ & $C 10_{9}^{0}$ & $C 10_{9}^{3}$ & $C 10_{9}^{5}$ & $C 11_{9}^{0}$ & $C 11_{9}^{3}$ & $C 11_{9}^{5}$ & $C 12_{9}^{1}$ \\
\hline$C 12_{9}^{2}$ & $C 12_{9}^{4}$ & $C 12_{9}^{5}$ & $C 13_{9}^{0}$ & $C 13_{9}^{1}$ & $C 13_{9}^{3}$ & $C 13_{9}^{6}$ & $C 14_{9}^{0}$ & $C 14_{9}^{1}$ & $C 14_{9}^{3}$ \\
\hline$C 14_{9}^{6}$ & $C 14_{9}^{7}$ & $C 15_{9}^{1}$ & $C 15_{9}^{2}$ & $C 15_{9}^{4}$ & $C 15_{9}^{5}$ & $C 15_{9}^{7}$ & $C 16_{9}^{0}$ & $C 16_{9}^{3}$ & $C 16_{9}^{4}$ \\
\hline
\end{tabular}


Table 11 (Continued)

\begin{tabular}{llllllllll}
\hline$C 16_{9}^{5}$ & $C 16_{9}^{6}$ & $C 16_{9}^{8}$ & $C 17_{9}^{0}$ & $C 17_{9}^{2}$ & $C 17_{9}^{3}$ & $C 17_{9}^{5}$ & $C 17_{9}^{6}$ & $C 17_{9}^{7}$ & $C 19_{9}^{0}$ \\
$C 19_{9}^{2}$ & $C 19_{9}^{3}$ & $C 19_{9}^{4}$ & $C 19_{9}^{6}$ & $C 19_{9}^{7}$ & $C 19_{9}^{8}$ & $C 20_{9}^{0}$ & $C 20_{9}^{3}$ & $C 20_{9}^{4}$ & $C 20_{9}^{5}$ \\
$C 20_{9}^{6}$ & $C 20_{9}^{7}$ & $C 20_{9}^{8}$ & $C 20_{9}^{10}$ & $C 3_{10}^{0}$ & $C 6_{10}^{8}$ & $C 7_{10}^{0}$ & $C 9_{10}^{0}$ & $C 9_{10}^{3}$ & $C 11_{10}^{0}$ \\
$C 11_{10}^{3}$ & $C 11_{10}^{4}$ & $C 12_{10}^{3}$ & $C 13_{10}^{0}$ & $C 13_{10}^{1}$ & $C 13_{10}^{2}$ & $C 13_{10}^{4}$ & $C 14_{10}^{1}$ & $C 14_{10}^{7}$ & $C 15_{10}^{1}$ \\
$C 15_{10}^{2}$ & $C 15_{10}^{3}$ & $C 15_{10}^{4}$ & $C 15_{10}^{6}$ & $C 15_{10}^{7}$ & $C 16_{10}^{1}$ & $C 16_{10}^{7}$ & $C 17_{10}^{0}$ & $C 17_{10}^{1}$ & $C 17_{10}^{2}$ \\
$C 17_{10}^{4}$ & $C 17_{10}^{6}$ & $C 17_{10}^{8}$ & $C 18_{10}^{3}$ & $C 18_{10}^{7}$ & $C 18_{10}^{9}$ & $C 19_{10}^{0}$ & $C 19_{10}^{2}$ & $C 19_{10}^{3}$ & $C 19_{10}^{4}$ \\
$C 19_{10}^{6}$ & $C 19_{10}^{7}$ & $C 19_{10}^{8}$ & & & & & & & \\
\hline
\end{tabular}

Table 12 A set of $R N_{n}^{v}$ symmetries for selection of homonuclear $J$-couplings $\{l, m, \lambda, \mu\}=\{0,0,0,0\}$ with suppression of all homonuclear DD couplings, CSA terms and isotropic shift terms. All inequivalent solutions in the range $N \leq 20, n \leq 10$ and $v \leq 10$ are shown

\begin{tabular}{llllllllll}
\hline$R 6_{1}^{0}$ & $R 8_{1}^{0}$ & $R 10_{1}^{0}$ & $R 12_{1}^{0}$ & $R 12_{1}^{3}$ & $R 14_{1}^{0}$ & $R 14_{1}^{3}$ & $R 14_{1}^{4}$ & $R 16_{1}^{0}$ & $R 16_{1}^{3}$ \\
$R 16_{1}^{4}$ & $R 16_{1}^{5}$ & $R 18_{1}^{0}$ & $R 18_{1}^{3}$ & $R 18_{1}^{4}$ & $R 18_{1}^{5}$ & $R 18_{1}^{6}$ & $R 20_{1}^{0}$ & $R 20_{1}^{3}$ & $R 20_{1}^{4}$ \\
$R 20_{1}^{5}$ & $R 20_{1}^{6}$ & $R 20_{1}^{7}$ & $R 6_{2}^{0}$ & $R 10_{2}^{0}$ & $R 12_{2}^{3}$ & $R 14_{2}^{0}$ & $R 16_{2}^{3}$ & $R 16_{2}^{5}$ & $R 18_{2}^{0}$ \\
$R 18_{2}^{3}$ & $R 18_{2}^{6}$ & $R 20_{2}^{3}$ & $R 20_{2}^{5}$ & $R 20_{2}^{7}$ & $R 8_{3}^{0}$ & $R 10_{3}^{0}$ & $R 14_{3}^{0}$ & $R 14_{3}^{2}$ & $R 14_{3}^{5}$ \\
$R 16_{3}^{0}$ & $R 16_{3}^{1}$ & $R 16_{3}^{4}$ & $R 16_{3}^{7}$ & $R 18_{3}^{1}$ & $R 18_{3}^{2}$ & $R 18_{3}^{4}$ & $R 18_{3}^{5}$ & $R 18_{3}^{7}$ & $R 18_{3}^{8}$ \\
$R 20_{3}^{0}$ & $R 20_{3}^{1}$ & $R 20_{3}^{2}$ & $R 20_{3}^{5}$ & $R 20_{3}^{8}$ & $R 20_{3}^{9}$ & $R 6_{4}^{0}$ & $R 10_{4}^{0}$ & $R 12_{4}^{1}$ & $R 12_{4}^{3}$ \\
$R 12_{4}^{5}$ & $R 14_{4}^{0}$ & $R 18_{4}^{0}$ & $R 18_{4}^{3}$ & $R 18_{4}^{6}$ & $R 20_{4}^{1}$ & $R 20_{4}^{3}$ & $R 20_{4}^{5}$ & $R 20_{4}^{7}$ & $R 20_{4}^{9}$ \\
$R 6_{5}^{0}$ & $R 8_{5}^{0}$ & $R 12_{5}^{0}$ & $R 12_{5}^{3}$ & $R 14_{5}^{0}$ & $R 14_{5}^{1}$ & $R 14_{5}^{6}$ & $R 16_{5}^{0}$ & $R 16_{5}^{1}$ & $R 16_{5}^{4}$ \\
$R 16_{5}^{7}$ & $R 18_{5}^{0}$ & $R 18_{5}^{2}$ & $R 18_{5}^{3}$ & $R 18_{5}^{6}$ & $R 18_{5}^{7}$ & & & & \\
\hline
\end{tabular}

Table 13 A set of $C N_{n}^{v}$ symmetries for selection of isotropic chemical shift terms. All CSA terms and homonuclear DD coupling terms are suppressed. The homonuclear $J$-coupling $\{0,0,0,0\}$ is symmetry-allowed. All inequivalent solutions in the range $N \leq 20, n \leq 10$ and $v \leq 10$ are shown

\begin{tabular}{|c|c|c|c|c|c|c|c|c|c|}
\hline$C 3_{1}^{0}$ & $C 4_{1}^{0}$ & $C 5_{1}^{0}$ & $C 6_{1}^{0}$ & $C 6_{1}^{3}$ & $C 7_{1}^{0}$ & $C 8_{1}^{0}$ & $C 8_{1}^{4}$ & $C 9_{1}^{0}$ & $C 9_{1}^{3}$ \\
\hline$C 10_{1}^{0}$ & $C 10_{1}^{3}$ & $C 10_{1}^{5}$ & $C 11_{1}^{3}$ & $C 11_{1}^{4}$ & $C 12_{1}^{3}$ & $C 12_{1}^{4}$ & $C 12_{1}^{6}$ & $C 13_{1}^{3}$ & $C 13_{1}^{4}$ \\
\hline$C 13_{1}^{5}$ & $C 14_{1}^{3}$ & $C 14_{1}^{4}$ & $C 14_{1}^{5}$ & $C 14_{1}^{7}$ & $C 15_{1}^{3}$ & $C 15_{1}^{4}$ & $C 15_{1}^{5}$ & $C 15_{1}^{6}$ & $C 16_{1}^{3}$ \\
\hline$C 16_{1}^{4}$ & $C 16_{1}^{5}$ & $C 16_{1}^{6}$ & $C 16_{1}^{8}$ & $C 17_{1}^{3}$ & $C 17_{1}^{4}$ & $C 17_{1}^{5}$ & $C 17_{1}^{6}$ & $C 17_{1}^{7}$ & $C 18_{1}^{3}$ \\
\hline$C 18_{1}^{4}$ & $C 18_{1}^{5}$ & $C 18_{1}^{6}$ & $C 18_{1}^{7}$ & $C 18_{1}^{9}$ & $C 19_{1}^{3}$ & $C 19_{1}^{4}$ & $C 19_{1}^{5}$ & $C 19_{1}^{6}$ & $C 19_{1}^{7}$ \\
\hline$C 19_{1}^{8}$ & $C 20_{1}^{3}$ & $C 20_{1}^{4}$ & $C 20_{1}^{5}$ & $C 20_{1}^{6}$ & $C 20_{1}^{7}$ & $C 20_{1}^{8}$ & $C 20_{1}^{10}$ & $C 3_{2}^{0}$ & $C 5_{2}^{0}$ \\
\hline$C 6_{2}^{3}$ & $C 7_{2}^{0}$ & $C 9_{2}^{0}$ & $C 9_{2}^{3}$ & $C 10_{2}^{5}$ & $C 11_{2}^{3}$ & $C 11_{2}^{5}$ & $C 12_{2}^{3}$ & $C 13_{2}^{3}$ & $C 13_{2}^{5}$ \\
\hline$C 13_{2}^{6}$ & $C 14_{2}^{3}$ & $C 14_{2}^{7}$ & $C 15_{2}^{3}$ & $C 15_{2}^{5}$ & $C 15_{2}^{6}$ & $C 15_{2}^{7}$ & $C 16_{2}^{3}$ & $C 16_{2}^{5}$ & $C 17_{2}^{3}$ \\
\hline$C 17_{2}^{5}$ & $C 17_{2}^{6}$ & $C 17_{2}^{7}$ & $C 17_{2}^{8}$ & $C 18_{2}^{3}$ & $C 18_{2}^{5}$ & $C 18_{2}^{9}$ & $C 19_{2}^{3}$ & $C 19_{2}^{5}$ & $C 19$ \\
\hline$C 19_{2}^{7}$ & $C 19_{2}^{8}$ & $C 19_{2}^{9}$ & $C 20_{2}^{3}$ & $C 20_{2}^{5}$ & $\mathrm{C} 2 \mathrm{O}_{2}^{7}$ & $C 4_{3}^{0}$ & $\mathrm{CS}_{3}^{0}$ & $C 7_{3}^{0}$ & $C 8_{3}^{0}$ \\
\hline$C 8_{3}^{4}$ & $C 9_{3}^{1}$ & $C 9_{3}^{2}$ & $C 9_{3}^{4}$ & $C 10_{3}^{0}$ & $C 10_{3}^{1}$ & $C 10_{3}^{5}$ & $C 11_{3}^{1}$ & $C 11_{3}^{2}$ & $C 12_{3}^{1}$ \\
\hline$C 12_{3}^{2}$ & $C 12_{3}^{4}$ & $C 12_{3}^{5}$ & $C 13_{3}^{1}$ & $C 13_{3}^{2}$ & $C 13_{3}^{4}$ & $C 14_{3}^{1}$ & $C 14_{3}^{2}$ & $C 14_{3}^{5}$ & $C 14_{3}^{7}$ \\
\hline$C 15_{3}^{1}$ & $C 15_{3}^{2}$ & $C 15_{3}^{4}$ & $C 15_{3}^{5}$ & $C 15_{3}^{7}$ & $C 16_{3}^{1}$ & $C 16_{3}^{2}$ & $C 16_{3}^{4}$ & $C 16_{3}^{7}$ & $C 16_{3}^{8}$ \\
\hline$C 17_{3}^{1}$ & $C 17_{3}^{2}$ & $C 17_{3}^{4}$ & $C 17_{3}^{5}$ & $C 17_{3}^{8}$ & $C 18_{3}^{1}$ & $C 18_{3}^{2}$ & $C 18_{3}^{4}$ & $C 18_{3}^{5}$ & $C 18_{3}^{7}$ \\
\hline$C 18_{3}^{8}$ & $C 19_{3}^{1}$ & $C 19_{3}^{2}$ & $C 19_{3}^{4}$ & $C 19_{3}^{5}$ & $C 19_{3}^{7}$ & $C 19_{3}^{9}$ & $C 20_{3}^{1}$ & $C 20_{3}^{2}$ & $C 20_{3}^{4}$ \\
\hline$C 20_{3}^{5}$ & $C 20_{3}^{8}$ & $C 20_{3}^{9}$ & $C 20_{3}^{10}$ & $C 3_{4}^{0}$ & $C 5_{4}^{0}$ & $C 6_{4}^{3}$ & $C 7_{4}^{0}$ & $C 9_{4}^{0}$ & $C 9_{4}^{3}$ \\
\hline$C 10_{4}^{5}$ & $C 11_{4}^{1}$ & $C 11_{4}^{5}$ & $C 12_{4}^{1}$ & $C 12_{4}^{3}$ & $C 12_{4}^{5}$ & $C 13_{4}^{1}$ & $C 13_{4}^{3}$ & $C 13_{4}^{6}$ & $C 14_{4}^{1}$ \\
\hline$C 14_{4}^{7}$ & $C 15_{4}^{1}$ & $C 15_{4}^{3}$ & $C 15_{4}^{5}$ & $C 15_{4}^{6}$ & $C 16_{4}^{1}$ & $C 16_{4}^{3}$ & $C 16_{4}^{5}$ & $C 16_{4}^{7}$ & $C 17_{4}^{1}$ \\
\hline$C 17_{4}^{3}$ & $C 17_{4}^{5}$ & $C 17_{4}^{6}$ & $C 17_{4}^{7}$ & $C 18_{4}^{1}$ & $C 18_{4}^{3}$ & $C 18_{4}^{9}$ & $C 19_{4}^{1}$ & $C 19_{4}^{3}$ & $C 19^{5}$ \\
\hline$C 19_{4}^{6}$ & $C 19_{4}^{7}$ & $C 19_{4}^{9}$ & $C 20_{4}^{1}$ & $C 20_{4}^{3}$ & $C 20_{4}^{5}$ & $C 20_{4}^{7}$ & $C 20_{4}^{9}$ & $C 3_{5}^{0}$ & $C 4_{5}^{0}$ \\
\hline$C 6_{5}^{0}$ & $C 6_{5}^{3}$ & $C 7_{5}^{0}$ & $C 8_{5}^{0}$ & $C 8_{5}^{4}$ & $C 9_{5}^{0}$ & $C 9_{5}^{3}$ & $C 11_{5}^{2}$ & $C 11_{5}^{4}$ & $C 12_{5}^{3}$ \\
\hline$C 12_{5}^{4}$ & $C 12_{5}^{6}$ & $C 13_{5}^{1}$ & $C 13_{5}^{2}$ & $C 13_{5}^{6}$ & $C 14_{5}^{1}$ & $C 14_{5}^{3}$ & $C 14_{5}^{6}$ & $C 14_{5}^{7}$ & $C 15_{5}^{1}$ \\
\hline$C 15_{5}^{2}$ & $C 15_{5}^{3}$ & $C 15_{5}^{4}$ & $C 15_{5}^{6}$ & $C 15_{5}^{7}$ & $C 16_{5}^{1}$ & $C 16_{5}^{2}$ & $C 16_{5}^{4}$ & $C 16_{5}^{7}$ & $C 16_{5}^{8}$ \\
\hline$C 17_{5}^{1}$ & $C 17_{5}^{2}$ & $C 17_{5}^{3}$ & $C 17_{5}^{4}$ & $C 17_{5}^{8}$ & $C 18_{5}^{1}$ & $C 18_{5}^{2}$ & $C 18_{5}^{3}$ & $C 18_{5}^{6}$ & $C 18_{5}^{7}$ \\
\hline$C 18_{5}^{9}$ & $C 19_{5}^{1}$ & $C 19_{5}^{2}$ & $C 19_{5}^{3}$ & $C 19_{5}^{4}$ & $C 19_{5}^{6}$ & $C 19_{5}^{8}$ & $C 20_{5}^{1}$ & $C 20_{5}^{2}$ & $C 20_{5}^{3}$ \\
\hline
\end{tabular}


Table 13 (Continued)

\begin{tabular}{|c|c|c|c|c|c|c|c|c|c|}
\hline$C 20_{5}^{4}$ & $C 20_{5}^{6}$ & $C 20_{5}^{7}$ & $C 20_{5}^{8}$ & $C 20_{5}^{9}$ & $C 5_{6}^{0}$ & $C 7_{6}^{0}$ & $C 9_{6}^{1}$ & $C 9_{6}^{2}$ & $C 9_{6}^{4}$ \\
\hline$C 10_{6}^{5}$ & $C 11_{6}^{2}$ & $C 11_{6}^{4}$ & $C 13_{6}^{2}$ & $C 13_{6}^{4}$ & $C 13_{6}^{5}$ & $C 14_{6}^{5}$ & $C 14_{6}^{7}$ & $C 15_{6}^{1}$ & $C 15_{6}^{2}$ \\
\hline$C 15_{6}^{4}$ & $C 15_{6}^{5}$ & $C 15_{6}^{7}$ & $C 16_{6}^{1}$ & $C 16_{6}^{7}$ & $C 17_{6}^{1}$ & $C 17_{6}^{2}$ & $C 17_{6}^{4}$ & $C 17_{6}^{7}$ & $C 17_{6}^{8}$ \\
\hline$C 18_{6}^{1}$ & $C 18_{6}^{5}$ & $C 18_{6}^{7}$ & $C 19_{6}^{1}$ & $C 19_{6}^{2}$ & $C 19_{6}^{4}$ & $C 19_{6}^{5}$ & $C 19_{6}^{8}$ & $C 19_{6}^{9}$ & $C 20_{6}^{1}$ \\
\hline$C 20_{6}^{5}$ & $C 20_{6}^{9}$ & $C 3_{7}^{0}$ & $C 4_{7}^{0}$ & $C 5_{7}^{0}$ & $C 6_{7}^{0}$ & $C 6_{7}^{3}$ & $C 8_{7}^{0}$ & $C 8_{7}^{4}$ & $C 9_{7}^{0}$ \\
\hline$C 9_{7}^{3}$ & $C 10_{7}^{0}$ & $C 10_{7}^{1}$ & $C 10_{7}^{5}$ & $C 11_{7}^{1}$ & $C 11_{7}^{5}$ & $C 12_{7}^{3}$ & $C 12_{7}^{4}$ & $C 12_{7}^{6}$ & $C 13_{7}^{2}$ \\
\hline$C 13_{7}^{4}$ & $C 13_{7}^{5}$ & $C 15_{7}^{2}$ & $C 15_{7}^{3}$ & $C 15_{7}^{5}$ & $C 15_{7}^{6}$ & $C 16_{7}^{3}$ & $C 16_{7}^{4}$ & $C 16_{7}^{5}$ & $C 16_{7}^{6}$ \\
\hline$C 16_{7}^{8}$ & $C 17_{7}^{1}$ & $C 17_{7}^{2}$ & $C 17_{7}^{4}$ & $C 17_{7}^{6}$ & $C 17_{7}^{8}$ & $C 18_{7}^{1}$ & $C 18_{7}^{3}$ & $C 18_{7}^{5}$ & $C 18_{7}^{6}$ \\
\hline$C 18_{7}^{8}$ & $C 18_{7}^{9}$ & $C 19_{7}^{1}$ & $C 19_{7}^{2}$ & $C 19_{7}^{3}$ & $C 19_{7}^{4}$ & $C 19_{7}^{8}$ & $C 19_{7}^{9}$ & $C 20_{7}^{1}$ & $C 20_{7}^{2}$ \\
\hline$C 20_{7}^{4}$ & $C 20_{7}^{5}$ & $C 20_{7}^{8}$ & $C 20_{7}^{9}$ & $C 20_{7}^{10}$ & $C 3_{8}^{0}$ & $C 5_{8}^{0}$ & $C 6_{8}^{3}$ & $C 7_{8}^{0}$ & $C 9_{8}^{0}$ \\
\hline$C 9_{8}^{3}$ & $C 10_{8}^{5}$ & $C 11_{8}^{1}$ & $C 11_{8}^{2}$ & $C 12_{8}^{1}$ & $C 12_{8}^{3}$ & $C 12_{8}^{5}$ & $C 13_{8}^{1}$ & $C 13_{8}^{2}$ & $C 13_{8}^{6}$ \\
\hline$C 14_{8}^{5}$ & $C 14_{8}^{7}$ & $C 15_{8}^{2}$ & $C 15_{8}^{3}$ & $C 15_{8}^{5}$ & $C 15_{8}^{6}$ & $C 17_{8}^{2}$ & $C 17_{8}^{3}$ & $C 17_{8}^{5}$ & $C 17_{8}^{6}$ \\
\hline$C 17_{8}^{7}$ & $C 18_{8}^{3}$ & $C 18_{8}^{7}$ & $C 18_{8}^{9}$ & $C 19_{8}^{1}$ & $C 19_{8}^{2}$ & $C 19_{8}^{5}$ & $C 19_{8}^{6}$ & $C 19_{8}^{7}$ & $C 19_{8}^{9}$ \\
\hline$C 20_{8}^{1}$ & $C 20_{8}^{3}$ & $C 20_{8}^{5}$ & $C 20_{8}^{7}$ & $C 20_{8}^{9}$ & $C 4_{9}^{0}$ & $C 5_{9}^{0}$ & $C 7_{9}^{0}$ & $C 8_{9}^{0}$ & $C 8_{9}^{4}$ \\
\hline$C 10_{9}^{0}$ & $C 10_{9}^{3}$ & $C 10_{9}^{5}$ & $C 11_{9}^{3}$ & $C 11_{9}^{5}$ & $C 12_{9}^{1}$ & $C 12_{9}^{2}$ & $C 12_{9}^{4}$ & $C 12_{9}^{5}$ & $C 13_{9}^{1}$ \\
\hline$C 13_{9}^{3}$ & $C 13_{9}^{6}$ & $C 14_{9}^{1}$ & $C 14_{9}^{3}$ & $C 14_{9}^{6}$ & $C 14_{9}^{7}$ & $C 15_{9}^{1}$ & $C 15_{9}^{2}$ & $C 15_{9}^{4}$ & $C 15_{9}^{5}$ \\
\hline$C 15_{9}^{7}$ & $C 16_{9}^{3}$ & $C 16_{9}^{4}$ & $C 16_{9}^{5}$ & $C 16_{9}^{6}$ & $C 16_{9}^{8}$ & $C 17_{9}^{2}$ & $C 17_{9}^{3}$ & $C 17_{9}^{5}$ & $C 17_{9}^{6}$ \\
\hline$C 17_{9}^{7}$ & $C 19_{9}^{2}$ & $C 19_{9}^{3}$ & $C 19_{9}^{4}$ & $C 19_{9}^{6}$ & $C 19_{9}^{7}$ & $C 19_{9}^{8}$ & $C 20_{9}^{3}$ & $C 20_{9}^{4}$ & $C 20_{9}^{5}$ \\
\hline$C 20_{9}^{6}$ & $C 20_{9}^{7}$ & $C 20_{9}^{8}$ & $C 20_{9}^{10}$ & $C 3_{10}^{0}$ & $C 6_{10}^{3}$ & $C 7_{10}^{0}$ & $C 9_{10}^{0}$ & $C 9_{10}^{3}$ & $C 11_{10}^{3}$ \\
\hline$C 11_{10}^{4}$ & $C 12_{10}^{3}$ & $C 13_{10}^{1}$ & $C 13_{10}^{2}$ & $C 13_{10}^{4}$ & $C 14_{10}^{1}$ & $C 14_{10}^{7}$ & $C 15_{10}^{1}$ & $C 15_{10}^{2}$ & $C 15_{10}^{3}$ \\
\hline$C 15_{10}^{4}$ & $C 15_{10}^{6}$ & $C 15_{10}^{7}$ & $C 16_{10}^{1}$ & $C 16_{10}^{7}$ & $C 17_{10}^{1}$ & $C 17_{10}^{2}$ & $C 17_{10}^{4}$ & $C 17_{10}^{6}$ & $C 17_{10}^{8}$ \\
\hline$C 18_{10}^{3}$ & $C 18_{10}^{7}$ & $C 18_{10}^{9}$ & $C 19_{10}^{2}$ & $C 19_{10}^{3}$ & $C 19_{10}^{4}$ & $C 19_{10}^{6}$ & $C 19_{10}^{7}$ & $C 19_{10}^{8}$ & \\
\hline
\end{tabular}

Table 14 A set of $R N_{n}^{v}$ symmetries for selection of isotropic chemical shift terms. In all cases, the terms $\{l, m, \lambda, \mu\}=\{0,0,1, \pm 1\}$ are selected. All CSA terms and homonuclear DD coupling terms are suppressed. The homonuclear $J$-coupling $\{0,0,0,0\}$ is symmetry-allowed. All inequivalent solutions in the range $N \leq 20, n \leq 10$ and $v \leq 10$ are shown

\begin{tabular}{llllllll}
\hline$R 6_{1}^{3}$ & $R 8_{1}^{4}$ & $R 10_{1}^{5}$ & $R 12_{1}^{6}$ & $R 14_{1}^{7}$ & $R 16_{1}^{8}$ & $R 18_{1}^{9}$ & $R 20_{1}^{10}$ \\
$R 6_{2}^{3}$ & $R 10_{2}^{5}$ & $R 14_{2}^{7}$ & $R 18_{2}^{9}$ & $R 8_{3}^{4}$ & $R 10_{3}^{5}$ & $R 14_{3}^{7}$ & $R 16_{3}^{8}$ \\
$R 20_{3}^{10}$ & $R 6_{4}^{3}$ & $R 10_{4}^{5}$ & $R 14_{4}^{7}$ & $R 18_{4}^{9}$ & $R 6_{5}^{3}$ & $R 8_{5}^{4}$ & $R 12_{5}^{6}$ \\
$R 14_{5}^{7}$ & $R 16_{5}^{8}$ & $R 18_{5}^{9}$ & & & & & \\
\hline
\end{tabular}

Table 15 A set of $R N_{n}^{v}$ symmetries for $\gamma$-encoded recoupling of CSA interactions, with selection of $\{l, m, \lambda, \mu\}=\{2, \pm 2,1, \pm 1\}$ or $\{2, \pm 2,1, \mp 1\}$ terms, and suppression of isotropic shift and homonuclear DD terms. The homonuclear $J$-coupling $\{0,0,0,0\}$ is also symmetry-allowed. The same solutions implement $\gamma$-encoded heteronuclear recoupling if they are applied to only one spin species. All inequivalent solutions in the range $N \leq 20, n \leq 10$ and $v \leq 10$ are shown

\begin{tabular}{llllllllll}
\hline$R 10_{1}^{3}$ & $R 12_{1}^{4}$ & $R 14_{1}^{5}$ & $R 16_{1}^{6}$ & $R 18_{1}^{7}$ & $R 20_{1}^{8}$ & $R 14_{2}^{3}$ & $R 18_{2}^{5}$ & $R 10_{3}^{1}$ & $R 14_{3}^{1}$ \\
$R 16_{3}^{2}$ & $R 20_{3}^{4}$ & $R 14_{4}^{1}$ & $R 18_{4}^{1}$ & $R 12_{5}^{4}$ & $R 14_{5}^{3}$ & $R 16_{5}^{2}$ & $R 18_{5}^{1}$ & $R 14_{6}^{5}$ & $R 10_{7}^{1}$ \\
$R 12_{7}^{4}$ & $R 16_{7}^{6}$ & $R 18_{7}^{5}$ & $R 20_{7}^{4}$ & $R 14_{8}^{5}$ & $R 18_{8}^{7}$ & $R 10_{9}^{3}$ & $R 14_{9}^{3}$ & $R 16_{9}^{6}$ & $R 20_{9}^{8}$ \\
$R 14_{10}^{1}$ & $R 18_{10}^{7}$ & & & & & & & & \\
\hline
\end{tabular}

Table 16 A set of $R N_{n}^{v}$ symmetries for non- $\gamma$-encoded zero-quantum recoupling of CSA interactions, with selection of $\{l, m, \lambda, \mu\}=\{2, \pm 2,1,0\}$ terms, and suppression of all isotropic shift and homonuclear DD terms. The homonuclear $J$-coupling $\{0,0,0,0\}$ is also symmetry-allowed. The same solutions implement zero-quantum heteronuclear recoupling if they are applied to only one spin species. All inequivalent solutions in the range $N \leq 20, n \leq 10$ and $v \leq 10$ are shown

\begin{tabular}{llllllllll}
\hline$R 12_{3}^{1}$ & $R 12_{3}^{2}$ & $R 12_{3}^{4}$ & $R 12_{3}^{5}$ & $R 16_{4}^{1}$ & $R 16_{4}^{3}$ & $R 16_{4}^{5}$ & $R 16_{4}^{7}$ & $R 20_{5}^{1}$ & $R 20_{5}^{2}$ \\
$R 20_{5}^{3}$ & $R 20_{5}^{4}$ & $R 20_{5}^{6}$ & $R 20_{5}^{7}$ & $R 20_{5}^{8}$ & $R 20_{5}^{9}$ & $R 12_{9}^{1}$ & $R 12_{9}^{2}$ & $R 12_{9}^{4}$ & $R 12_{9}^{5}$ \\
\hline
\end{tabular}


element $\mathcal{R}=90_{180} 270_{0}$. An experimental correlation spectrum of $\left[\mathrm{U}-{ }^{13} \mathrm{C}\right]$-L-tyrosine at a spinning frequency of $20 \mathrm{kHz}$ is shown in Figure 10.

\subsection{Homonuclear Single-Quantum Recoupling}

Table 10 shows a set of symmetries for recoupling the $\mu=$ \pm 1 components of the homonuclear DD interaction. These symmetries are expected to be useful in multiple-quantum spin counting experiments. ${ }^{\mathbf{9 3 , 9 4 , 1 0 9 , 1 1 0}}$

\subsection{Selection of $\boldsymbol{J}$-Couplings}

Compilations of symmetries appropriate for the selection of homonuclear $J$-couplings $\{l, m, \lambda, \mu\}=\{0,0,0,0\}$ with suppression of homonuclear DD couplings and chemical shift anisotropies are given in Tables 11 and 12. The $R N_{n}^{v}$ symmetries in Table 12 also suppress isotropic chemical shift terms. For the $C N_{n}^{v}$ symmetries in Table 11, on the other hand, the scaling factors for the isotropic shift terms must be set to zero by an appropriate choice of $\mathcal{C}$.

These symmetries may be used in through-bond correlation spectroscopy (TOBSY) experiments. ${ }^{4-52}$ The pulse sequence is used as the mixing interval in a two-dimensional experiment; The transfer of longitudinal magnetization generates cross peaks in the $2 \mathrm{D}$ spectrum, indicating spin sites with finite mutual $J$-couplings (see Through-Bond Experiments in Solids).

The original pulse sequences of this kind, suggested by Baldus and Meier ${ }^{\mathbf{4 8 - 5 2}}$ have symmetries $R 6_{1}^{0}$ and $R 8_{1}^{0}$. Two groups $^{\mathbf{5 0 , 5 1}}$ have proposed a new range of pulse sequence, operational at higher spinning frequencies, which employ symmetries such as $C 9_{3}^{1}, C 9_{6}^{1}$ and others included in Table 11 . Chan has demonstrated a $R 30_{6}^{14}$ sequence which also has good performance at high spinning frequency. ${ }^{\mathbf{5 2}}$

\subsection{Selection of Isotropic Chemical Shifts}

High-resolution isotropic shift spectra may be obtained in solids if all DD couplings and CSA terms are suppressed. In principle, this may be done by sufficiently rapid sample spinning. However, in practice, the required spinning frequencies are usually impractical. A well-designed rf pulse sequence may assist the spatial averaging, leading to acceptable resolution at practical spinning frequencies. The most common applications of such CRAMPS methods are in the high-resolution ${ }^{1} \mathrm{H}$ NMR of organic powdered solids (see Line Narrowing Methods in Solids, Volume 4).

Tables 13 and 14 show symmetry solutions for fastspinning CRAMPS. All these symmetries suppress the homonuclear DD couplings and the CSA interactions, while allowing some isotropic shift terms. In this application it is particularly important that the scaling factors for the isotropic shift terms are as large as possible. This requires careful design of the element $\mathcal{C}$.

Spiess and co-workers employed $C 3_{1}^{0}$ and $C 4_{1}^{0}$ symmetries in fast-spinning CRAMPS experiments, using the known WHH4 cycles $^{\mathbf{1 2 , 1 3}}$ as the basic element $\mathcal{C}^{\mathbf{4 6 , 4 7}}$ Recently, Madhu and Zhao demonstrated the use of $R 18_{2}^{9}$ symmetry. ${ }^{111}$ Figure 11 shows an experimental ${ }^{1} \mathrm{H}$ spectrum obtained at a spinning frequency of $20 \mathrm{kHz}$ with a $R 18_{2}^{9}$ sequence

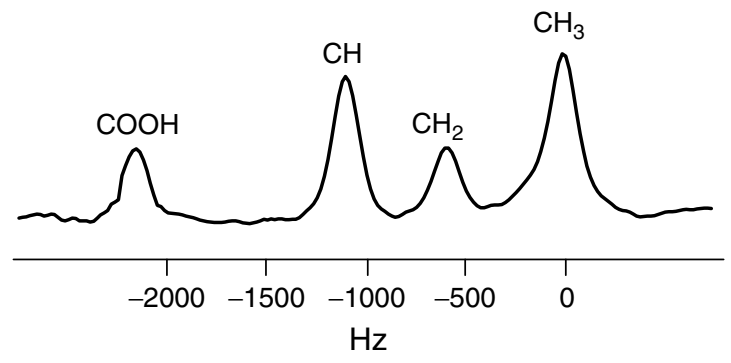

Figure $11{ }^{1} \mathrm{H}$ spectrum of monoethylfumarate, obtained at a field of $9.4 \mathrm{~T}$ and a spinning frequency of $20 \mathrm{kHz}$ with a $R 18_{2}^{9}$ sequence. After correcting for the pulse sequence scaling factor, the linewidths of all four peaks are given by approximately $0.9 \mathrm{ppm}$. (Adapted from Ref. ${ }^{111}$ )

constructed from basic elements $\mathcal{R}=90_{-45} 90_{45} 90_{-45}$. Note the use of a phase-modulated basic element, in order to enhance the local averaging of the strong homonuclear interactions.

\subsection{CSA Recoupling}

Table 15 shows a set of symmetries leading to $\gamma$-encoded recoupling of CSA interactions, with suppression of isotropic chemical shifts and homonuclear DD couplings. The recoupled average Hamiltonian is transverse, i.e., it has the form $I_{x} \cos \phi+I_{y} \sin \phi$.

These sequences could be useful for measuring the CSA principal values of individual sites in coupled spin clusters.

Table 16 shows a set of symmetries leading to recoupling of the zero-quantum $(\mu=0)$ part of the CSA interaction, with suppression of isotropic chemical shifts and homonuclear DD couplings. In this case, the recoupling is not $\gamma$-encoded, and the recoupled average Hamiltonian is longitudinal, i.e., proportional to the operator $I_{z}$.

\section{7 $\gamma$-Encoded Heteronuclear Recoupling}

The solutions in Table 15 may also be used for the selective $\gamma$-encoded recoupling of heteronuclear DD interactions. If these sequences are applied repetitively at the Larmor frequency of one spin species, while the NMR signals from a coupled spin species are observed, the resulting spectrum displays a splitting, due to the recoupled heteronuclear interactions. Since the recoupling is $\gamma$-encoded, the splittings are prominent, even in orientationally-disordered systems such as powders.

Zhao has demonstrated the measurement of ${ }^{13} \mathrm{C}-{ }^{1} \mathrm{H}$ and ${ }^{15} \mathrm{~N}-{ }^{1} \mathrm{H}$ DD couplings, using sequences with $R 18_{1}^{7}$ and $R 18_{2}^{5}$ symmetries. ${ }^{112,113}$ An experimental example is shown in Figure 12. The figure shows the individual ${ }^{15} \mathrm{~N}$ spectra of the two different $\mathrm{NH}$ groups in the imidazole ring of Lhistidine hydrochloride monohydrate, obtained in a $2 \mathrm{D}$ experiment in which a $R 18_{2}^{5}$ sequence is applied at the ${ }^{1} \mathrm{H}$ Larmor frequency. ${ }^{113}$ There is a visible difference in the two splittings, which corresponds to a $4 \mathrm{pm}$ difference in the two $\mathrm{NH}$ bond lengths. This difference is attributed to an intermolecular hydrogen bond formed by one of the $\mathrm{NH}$ groups. This result shows the potential of recoupling experiments for investigating the details of intermolecular interactions and other bonding 
Table 17 A set of $C R N_{n}^{v_{I}, v_{S}}$ symmetries for $\gamma$-encoded double-quantum heteronuclear recoupling, with suppression of all CSA terms, isotropic chemical shift terms, homonuclear DD terms, and heteronuclear $J$-couplings. All symmetries recouple terms of the form $\left\{l, m, \lambda_{I}, \mu_{I}, \lambda_{S}, \mu_{S}\right\}=$ $\{2, \mp 1,1, \pm 1,1, \pm 1\}$ The homonuclear $J$-couplings $\{0,0,0,0,0,0\}$ are symmetry-allowed. All in-equivalent solutions in the range $N \leq 20, n \leq 9$ and $N / n \leq 7$ are shown

\begin{tabular}{llllll}
\hline$C R 14_{3}^{2,2}$ & $C R 14_{3}^{-5,-5}$ & $C R 16_{3}^{1,4}$ & $C R 16_{3}^{-4,-7}$ & $C R 18_{3}^{-1,7}$ & $C R 18_{3}^{1,5}$ \\
$C R 18_{3}^{-2,8}$ & $C R 18_{3}^{2,4}$ & $C R 18_{3}^{-4,-8}$ & $C R 18_{3}^{-5,-7}$ & $C R 20_{3}^{-1,8}$ & $C R 20_{3}^{-2,9}$ \\
$C R 20_{3}^{2,5}$ & $C R 20_{3}^{-5,-8}$ & $C R 20_{4}^{-1,7}$ & $C R 20_{4}^{1,5}$ & $C R 20_{4}^{-3,9}$ & $C R 20_{4}^{3,3}$ \\
$C R 20_{4}^{-5,-9}$ & $C R 20_{4}^{-7,-7}$ & $C R 14_{5}^{1,1}$ & $C R 14_{5}^{-6,-6}$ & $C R 16_{5}^{-1,4}$ & $C R 16_{5}^{-4,7}$ \\
$C R 18_{5}^{-2,6}$ & $C R 18_{5}^{2,2}$ & $C R 18_{5}^{-3,7}$ & $C R 18_{5}^{-7,-7}$ & $C R 16_{6}^{1,1}$ & $C R 16_{6}^{-7,-7}$ \\
$C R 20_{6}^{-1,5}$ & $C R 20_{6}^{-5,9}$ & $C R 16_{7}^{-3,4}$ & $C R 16_{7}^{-4,5}$ & $C R 18_{7}^{-1,3}$ & $C R 18_{7}^{1,1}$ \\
$C R 18_{7}^{-6,8}$ & $C R 18_{7}^{-8,-8}$ & $C R 20_{7}^{1,2}$ & $C R 20_{7}^{-2,5}$ & $C R 20_{7}^{-5,8}$ & $C R 20_{7}^{-8,-9}$ \\
$C R 20_{8}^{-1,3}$ & $C R 20_{8}^{1,1}$ & $C R 20_{8}^{-3,5}$ & $C R 20_{8}^{-5,7}$ & $C R 20_{8}^{-7,9}$ & $C R 20_{8}^{-9,-9}$ \\
$C R 14_{9}^{-1,-1}$ & $C R 14_{9}^{6,6}$ & $C R 16_{9}^{3,-4}$ & $C R 16_{9}^{4,-5}$ & $C R 20_{9}^{-3,4}$ & $C R 20_{9}^{-4,5}$ \\
$C R 20_{9}^{-5,6}$ & $C R 20_{9}^{-6,7}$ & & & & \\
\hline
\end{tabular}

Table 18 A set of $R N_{n}^{v_{I}, v_{S}}$ symmetries for $\gamma$-encoded double-quantum heteronuclear recoupling, with suppression of all CSA terms, isotropic chemical shift terms and homonuclear DD terms. All symmetries recouple terms of the form $\left\{l, m, \lambda_{I}, \mu_{I}, \lambda_{S}, \mu_{S}\right\}=\{2, \mp 1,1, \pm 1,1, \pm 1\}$ The homonuclear $J$-couplings $\{0,0,0,0,0,0\}$ and heteronuclear $J$-coupling $\{0,0,1,0,1,0\}$ are symmetry-allowed. All inequivalent solutions in the range $N \leq 20, n \leq 9$ and $N / n \leq 7$ are shown

\begin{tabular}{llllll}
\hline$R 14_{3}^{2,-5}$ & $R 16_{3}^{1,-4}$ & $R 16_{3}^{4,-7}$ & $R 18_{3}^{-1,-2}$ & $R 18_{3}^{1,-4}$ & $R 18_{3}^{2,-5}$ \\
$R 18_{3}^{4,-7}$ & $R 18_{3}^{5,-8}$ & $R 18_{3}^{7,8}$ & $R 20_{3}^{-1,-2}$ & $R 20_{3}^{2,-5}$ & $R 20_{3}^{5,-8}$ \\
$R 20_{3}^{8,9}$ & $R 20_{4}^{-1,-3}$ & $R 20_{4}^{1,-5}$ & $R 20_{4}^{3,-7}$ & $R 20_{4}^{5,-9}$ & $R 20_{4}^{7,9}$ \\
$R 14_{5}^{1,-6}$ & $R 16_{5}^{-1,-4}$ & $R 16_{5}^{4,7}$ & $R 18_{5}^{-2,-3}$ & $R 18_{5}^{2,-}-7$ & $R 18_{5}^{6,7}$ \\
$R 16_{6}^{1,-7}$ & $R 20_{6}^{-1,-5}$ & $R 20_{6}^{5,9}$ & $R 16_{7}^{-3,-4}$ & $R 16_{7}^{4,5}$ & $R 18_{7}^{-1,-6}$ \\
$R 18_{7}^{1,-8}$ & $R 18_{7}^{3,8}$ & $R 20_{7}^{1,-8}$ & $R 20_{7}^{-2,-5}$ & $R 20_{7}^{2,-9}$ & $R 20_{7}^{5,8}$ \\
$R 20_{8}^{-1,-7}$ & $R 20_{8}^{1,-9}$ & $R 20_{8}^{-3,-5}$ & $R 20_{8}^{3,9}$ & $R 20_{8}^{5,7}$ & $R 14_{9}^{1,-6}$ \\
$R 16_{9}^{3,4}$ & $R 16_{9}^{-4,-5}$ & $R 20_{9}^{-3,-6}$ & $R 20_{9}^{-4,-5}$ & $R 20_{9}^{4,7}$ & $R 20_{9}^{5,6}$ \\
\hline
\end{tabular}

perturbations, in systems that are unsuitable for crystallography or solution NMR.

The symmetry $C 5_{1}^{1}$ has also been used for $\gamma$-encoded heteronuclear recoupling, although it does not fully suppress the homonuclear DD terms. ${ }^{114-116}$ The performance of $C 5_{1}^{1}$ is acceptable if it is used together with a basic element $\mathcal{C}$ with good local averaging properties for the homonuclear DD interactions. The frequency-switched Lee-Goldburg (FSLG) ${ }^{\mathbf{1 7}}$ and MREV $^{14,15}$ sequences have both been used.14-116 Nevertheless, one expects some interference from the homonuclear DD couplings in the $C 5_{1}^{1}$-based methods.

\subsection{Non- $\boldsymbol{\gamma}$-Encoded Heteronuclear Recoupling}

The symmetries in Table 16 lead to zero-quantum recoupling of heteronuclear DD interactions, with suppression of chemical shift terms and homonuclear DD couplings. The recoupled heteronuclear interactions are proportional to the operator product $I_{z} S_{z}$.

If the $R N_{n}^{v}$ sequence is applied to the $I$-spin species, the $S$-spin NMR signals display a splitting which may be used to estimate the magnitude of the heteronuclear DD coupling. Since the recoupling is not $\gamma$-encoded, the splittings are relatively obscure in a powder sample.

Although the CSA interactions of resonant spins are recoupled at the same time as the heteronuclear DD interactions, the influence of the recoupled CSA terms is often minor, since the terms commute. In the single-quantum case of Table 15 , on the other hand, the two recoupled interactions do not necessarily commute, and may interfere with each other in a complex way. The solutions in Table 15 are therefore only suitable for the heteronuclear recoupling of spins with small CSA interactions, while the solutions in Table 16 are more generally applicable.

The symmetries in Table 16 may be regarded as extensions of the REDOR pulse sequence. ${ }^{\mathbf{2 6 , 2 7}}$ Unlike REDOR, they implement homonuclear dipolar decoupling of the irradiated spin species, and should be applicable to systems with homonuclear dipolar interactions.

REDOR itself consists of two $180^{\circ}$ pulses per rotor period, and therefore belongs to the symmetry class $R N_{N / 2}^{v}$. The most common implementation of REDOR follows the XY8 phase scheme, which corresponds to the supercycle $\mathcal{S S}^{\prime}$ of the symmetry $R 4_{2}^{1}$. This symmetry implements selection of the $\{l, m, \lambda, \mu\}=\{2, \pm 1,1,0\}$ terms, just as the solutions in Table 16 . However, the $R 4_{2}^{1}$ symmetry also allows homonuclear DD terms of the form $\{2, \pm 2,2,0\},\{2, \pm 1,2, \pm 2\}$ and $\{2, \pm 1,2, \mp 2\}$. These recoupled homonuclear DD interactions interfere with the operation of REDOR in non-dilute spin systems, a defect which should be reduced by the symmetries in Table 16.

Chan has discovered a different way of suppressing the homonuclear DD interactions in the REDOR method. ${ }^{67}$ The 


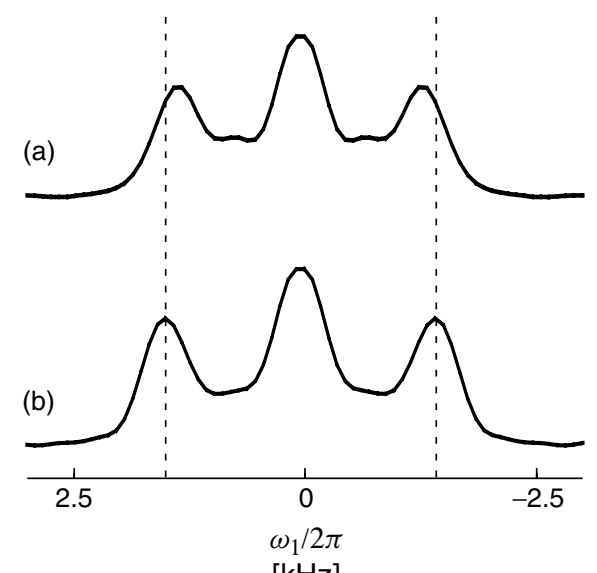

$[\mathrm{kHz}]$

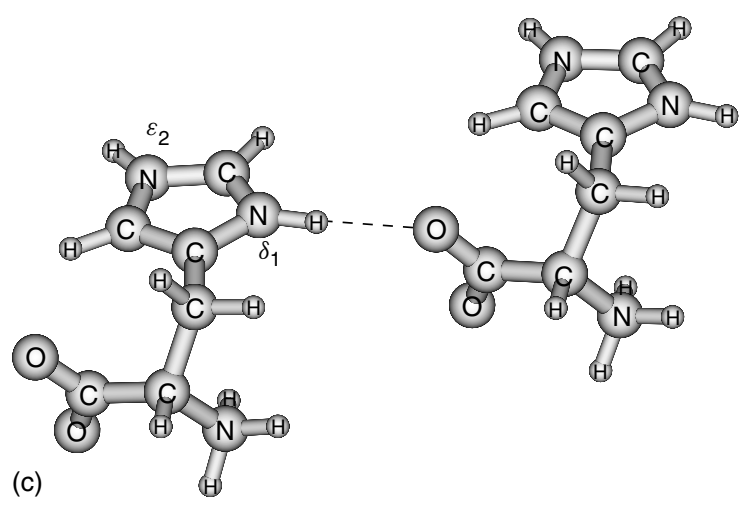

Figure 12 (a) Experimental ${ }^{15} \mathrm{~N}$ spectrum of the $\delta_{1}$ nitrogen site of $\left[\mathrm{U}-{ }^{13} \mathrm{C},{ }^{15} \mathrm{~N}\right]$-L-histidinium hydrochloride monohydrate, obtained by a $2 \mathrm{D}$ experiment in the presence of $R 18_{2}^{5}$ irradiation of the protons, at a magnetic field of $9.4 \mathrm{~T}$ and a spinning frequency of $20 \mathrm{kHz}$. The three-peak structure is due to the recoupled heteronuclear interaction between the ${ }^{15} \mathrm{~N}$ and the directly-bonded ${ }^{1} \mathrm{H}$. (b) Experimental ${ }^{15} \mathrm{~N}$ spectrum of the $\varepsilon_{2}$ nitrogen site. Note that the splitting is slightly larger than in plot (a). These spectra show that the $\delta_{1} \mathrm{NH}$ bond is about $4 \mathrm{pm}$ longer than the $\varepsilon_{2} \mathrm{NH}$ bond. This elongation is attributed to the existence of an intermolecular hydrogen bond (see crystal structure in (c)). (Adapted from Ref. ${ }^{\mathbf{1 1 3}}$ )

C-REDOR sequences are based upon the symmetries $C 3_{3}^{1}$ and $C 7_{7}^{1}$. These symmetries allow heteronuclear DD terms of the form $\{l, m, \lambda, \mu\}=\{2, \pm 1,1,0\}$ and $\{2, \pm 2,1,0\}$, and also the isotropic chemical shift term $\{0,0,1,0\}$ and homonuclear DD terms of the form $\{2, \pm 1,2,0\}$ and $\{2, \pm 2,2,0\}$. This does not seem very promising since most of these terms are undesirable. However, by using the POST element $\mathcal{C}=90_{0} 360_{180} 270_{0}$, the scaling factors for all of the symmetry-allowed terms with the exception of $\{2, \pm 1,1,0\}$ and $\{0,0,0,0\}$ may be made to vanish. The result is a REDOR-like average Hamiltonian with suppression of homonuclear DD couplings. This has been demonstrated experimentally. ${ }^{67}$

\subsection{Double-Quantum Heteronuclear Recoupling by Dual Pulse Sequences}

It is also possible to recouple heteronuclear interactions by applying synchronized rf irradiation to both spin species. This procedure allows $\gamma$-encoded recoupling of the heteronuclear interactions with the suppression of CSA interference.
The 'dual' selection rules in equations (58)-(61) may be used to generate the symmetry solutions. Sets of symmetries suitable for $\gamma$-encoded double-quantum heteronuclear recoupling are shown in Table 17 and 18. All of these symmetries recouple the terms of the form $\left\{l, m, \lambda_{I}, \mu_{I}, \lambda_{S}, \mu_{S}\right\}=$ $\{2, \mp 1,1, \pm 1,1, \pm 1\}$ with suppression of all isotropic chemical shift, homonuclear DD coupling and CSA terms. In the case of the $C R N_{n}^{v_{I}, v_{S}}$ symmetries in Table 17, the heteronuclear $J$-couplings are also suppressed.

Brinkmann ${ }^{\mathbf{6 0}}$ has shown that these symmetries may be used for the generation of heteronuclear multiple-quantum coherences, for the measurement of heteronuclear coupling constants, and for implementing magnetization transfer between spin species. For example, a sequence with the symmetry $R 24_{9}^{5,10}$ has been used to obtain a heteronuclear multiplequantum spectrum of a labelled amino acid. ${ }^{\mathbf{6 0}}$

\subsection{Heteronuclear Decoupling}

The two-pulse phase modulation (TPPM) decoupling method ${ }^{\mathbf{1 0}}$ may also be understood qualitatively using the symmetry theory. The TPPM modulation scheme corresponds to a $R N_{n}^{v}$ sequence with a large ratio of $N$ to $n$, although exact synchronization with the sample rotation does not appear to be important in this case. Edén has shown ${ }^{\mathbf{4 1 , 4 5}}$ that in many cases, a similar decoupling quality to TPPM may be achieved with exactly synchronized $C N_{n}^{v}$ and $R N_{n}^{v}$ sequences, and that the decoupling quality correlates reasonably well with higher-order term counts obtained by applying the rules in equations (31) and (32). In addition, there is evidence that the quality of heteronuclear decoupling is enhanced by implementing homonuclear recoupling at the same time, using symmetries such as $R 24_{2}^{1}{ }^{41}$ This actually happens inadvertently in many implementations of TPPM. This observation is consistent with the well-known effect of 'self-decoupling', where strong homonuclear couplings within one spin species can narrow the peaks of a second, coupled, spin species. ${ }^{4}$ (See Heteronuclear Decoupling in Solids.)

\section{CONCLUSIONS}

The symmetry theory sketched here is still in its infancy, although it is already clear that it provides much insight into the operation of many existing pulse sequences, as well as many new experimental possibilities. There remain many theoretical problems, such as the treatment of higher-order terms, and many practical problems, such as the phase-shift sensitivity of the $R N_{n}^{v}$ sequences. It is hoped that this summary will encourage many other research groups to explore the subject.

\section{REFERENCES}

1. E. R. Andrew, A. Bradbury, and R. G. Eades, Nature, 1959, 183, 1802.

2. I. J. Lowe, Phys. Rev. Lett., 1959, 2, 285.

3. E. O. Stejskal, J. Schaefer, and J. S. Waugh, J. Magn. Reson., 1977, 28, 105.

4. M. Mehring, 'High Resolution NMR in Solids', 2nd edn., Springer: Berlin, 1982. 
5. A. Abragam, 'The Principles of Nuclear Magnetism', Clarendon Press: Oxford, 1961.

6. R. R. Ernst, J. Chem. Phys., 1966, 45, 3845.

7. M. H. Levitt and R. Freeman, J. Magn. Reson., 1981, 43, 502.

8. J. S. Waugh, J. Magn. Reson., 1982, 49, 517.

9. M. H. Levitt, R. Freeman, and T. A. Frenkiel, Adv. Magn. Reson., 1983, 11, 47.

10. A. E. Bennett, C. M. Rienstra, M. Auger, K. V. Lakshmi, and R. G. Griffin, J. Chem. Phys., 1995, 103, 1.

11. M. Lee and W. I. Goldburg, Phys. Rev. A, 1965, 140, 1261.

12. J. S. Waugh, M. L. Huber, and U. Haeberlen, Phys. Rev. Lett., 1968, 20, 180.

13. U. Haeberlen and J. S. Waugh, Phys. Rev., 1968, 175, 453.

14. P. Mansfield, J. Phys. C, 1971, 4, 1444.

15. W. K. Rhim, D. D. Elleman, L. B. Schreiber, and R. W. Vaughan, J. Chem. Phys., 1974, 60, 4595.

16. D. P. Burum, M. Linder, and R. R. Ernst, J. Magn. Reson., 1981, 44, 173.

17. A. Bielecki, A. C. Kolbert, and M. H. Levitt, Chem. Phys. Lett., 1989, 155, 341.

18. M. Hohwy and N. C. Nielsen, J. Chem. Phys., 1997, 106, 1.

19. B. C. Gerstein, R. G. Pembleton, R. D. Wilson, and L. J. Ryan, J. Chem. Phys., 1977, 66, 361.

20. G. E. Maciel, C. E. Bronnimann, and B. Hawkins, Adv. Magn. Reson., 1990, 14, 125.

21. W. T. Dixon, J. Chem. Phys., 1982, 77, 1800.

22. O. N. Antzutkin, Z. Song, X. Feng, and M. H. Levitt, J. Chem. Phys., 1994, 100, 130.

23. Y. Yarim-Agaev, P. N. Tutunjian, and J. S. Waugh, J. Magn. Reson., 1982, 47, 51.

24. T. G. Oas, R. G. Griffin, and M. H. Levitt, J. Chem. Phys., 1988, 89, 692.

25. M. H. Levitt, T. G. Oas, and R. G. Griffin, Isr. J. Chem., 1988, 28, 271.

26. T. Gullion and J. Schaefer, J. Magn. Reson., 1989, 81, 196-200.

27. T. Gullion and J. Schaefer, Adv. Magn. Reson., 1989, 13, 57.

28. R. Tycko, Phys. Rev. Lett., 1988, 60, 2734.

29. R. Tycko, J. Chem. Phys., 1990, 92, 5776.

30. R. Tycko and G. Dabbagh, Chem. Phys. Lett., 1990, 173, 461.

31. R. Tycko and G. Dabbagh, J. Am. Chem. Soc., 1991, 113, 9444.

32. R. Tycko and S. O. Smith, J. Chem. Phys., 1993, 98, 932.

33. A. E. Bennett, J. H. Ok, R. G. Griffin, and S. Vega, J. Chem. Phys., 1992, 96, 8624.

34. D. K. Sodickson, M. H. Levitt, S. Vega, and R. G. Griffin, J. Chem. Phys., 1993, 98, 6742.

35. N. C. Nielsen, H. Bildsøe, H. J. Jakobsen, and M. H. Levitt, J. Chem. Phys., 1994, 101, 1805.

36. Y. K. Lee, N. D. Kurur, M. Helmle, O. G. Johannessen, N. C. Nielsen, and M. H. Levitt, Chem. Phys. Lett., 1995, 242, 304.

37. M. Hohwy, H. J. Jakobsen, M. Edén, M. H. Levitt, and N. C. Nielsen, J. Chem. Phys., 1998, 108, 2686.

38. C. M. Rienstra, M. E. Hatcher, L. J. Mueller, B. Sun, S. W. Fesik, and R. G. Griffin, J. Am. Chem. Soc., 1998, 120, $10602-10612$.

39. A. Brinkmann, M. Edén, and M. H. Levitt, J. Chem. Phys., 2000, 112, 8539-8554.

40. M. Hohwy, C. M. Rienstra, C. P. Jaroniec, and R. G. Griffin, J. Chem. Phys., 1999, 110, 7983-7992.

41. M. Carravetta, M. Edén, X. Zhao, A. Brinkmann, and M. H. Levitt, Chem. Phys. Lett., 2000, 321, 205-215.

42. A. Brinkmann, M. Carravetta, X. Zhao, M. Edén, J. Schmedt auf der Günne, and M. H. Levitt, in 'Perspectives on Solid State NMR in Biology', eds S. Kühne and H. J. M. de Groot, Kluwer: Dordrecht, 2001.

43. X. Feng, P. J. E. Verdegem, Y. K. Lee, D. Sandström, M. Edén, P. Bovee-Geurts, W. J. de Grip, J. Lugtenburg, H. J. M. de Groot, and M. H. Levitt, J. Am. Chem. Soc., 1997, 119, 6853-6857.
44. X. Feng, P. J. E. Verdegem, M. Edén, D. Sandström, Y. K. Lee, P. Bovee-Geurts, W. J. de Grip, J. Lugtenburg, H. J. M. de Groot, and M. H. Levitt, J. Biomol. NMR, 2000, 16, 1-8.

45. M. Edén and M. H. Levitt, J. Chem. Phys., 1999, 111, $1511-1519$

46. D. E. Demco, S. Hafner, and H. W. Spiess, J. Magn. Reson. A, 1995, 116, 36

47. S. Hafner and H. W. Spiess, J. Magn. Reson. A, 1996, 121, 160. 48. M. Baldus and B. H. Meier, J. Magn. Reson. A, 1996, 121, 65.

49. M. Baldus, R. J. Iulicci, and B. H. Meier, J. Am. Chem. Soc., 1997, 119, 1121-1124.

50. E. H. Hardy, R. Verel, and B. H. Meier, J. Magn. Reson., 2001, 148, 459-464.

51. A. S. D. Heindrichs, H. Geen, C. Giordani, and J. J. Titman, Chem. Phys. Lett., 2001, 335, 89-96.

52. J. C. C. Chan and G. Brunklaus, in press.

53. D. A. Varshalovich, A. N. Moskalev, and V. K. Kheronskii, 'Quantum Theory of Angular Momentum', World Scientific: Singapore, 1988.

54. M. H. Levitt, J. Magn. Reson., 1989, 82, 427.

55. Z. Song, O. N. Antzutkin, Y. K. Lee, S. C. Shekar, A. Rupprecht, and M. H. Levitt, Biophys. J., 1997, 73, 1539.

56. L. van Dam and M. H. Levitt, J. Mol. Biol., 2000, 304, $541-560$.

57. A. Samoson, E. Lippmaa, and A. Pines, Mol. Phys., 1988, 65, 1013.

58. A. Llor and J. Virlet, Chem. Phys. Lett., 1988, 152, 248.

59. B. F. Chmelka and A. Pines, Science, 1989, 246, 71.

60. A. Brinkmann and M. H. Levitt, J. Chem. Phys., 2001, 115, 357-384.

61. M. H. Levitt, Prog. NMR Spectrosc., 1986, 18, 61.

62. M. H. Levitt and R. Freeman, J. Magn. Reson., 1981, 43, 65.

63. S. Wolfram, 'Mathematica: A System for Doing Mathematics by Computer', Addison-Wesley: New York, 1991.

64. Y. Ishii, J. Chem. Phys., 2001, 114, 8473-8483.

65. A. Brinkmann, J. Schmedt auf der Günne, and M. H. Levitt, to be published.

66. B. -Q. Sun, P. R. Costa, D. Kocisko, P. T. Lansbury, and R. G. Griffin, J. Chem. Phys., 1995, 102, 702.

67. J. C. C. Chan, Chem. Phys. Lett., 2001, 335, 289-297.

68. H. Geen, J. J. Titman, J. Gottwald, and H. W. Spiess, J. Magn. Reson. A, 1995, 114, 264.

69. R. Graf, D. E. Demco, S. Hafner, and H. W. Spiess, Solid State Nucl. Magn. Reson., 1998, 12, 139.

70. D. P. Raleigh, M. H. Levitt, and R. G. Griffin, Chem. Phys. Lett., 1988, 146, 71.

71. M. H. Levitt, D. P. Raleigh, F. Creuzet, and R. G. Griffin, J. Chem. Phys., 1990, 92, 6347.

72. M. Helmle, Y. K. Lee, P. J. E. Verdegem, X. Feng, T. Karlsson, J. Lugtenburg, H. J. M. de Groot, and M. H. Levitt, J. Magn. Reson., 1999, 140, 379-403.

73. G. S. Harbison and H. W. Spiess, Chem. Phys. Lett., 1986, 124, 128.

74. P. Tang, C. -L. Juang, and G. S. Harbison, Science, 1990, 249, 70.

75. C. Glaubitz and A. Watts, J. Magn. Reson., 1998, 130, 305.

76. X. Feng, Y. K. Lee, D. Sandström, M. Edén, H. Maisel, A. Sebald, and M. H. Levitt, Chem. Phys. Lett., 1996, 257, 314.

77. S. Ravindranathan, X. Feng, T. Karlsson, G. Widmalm, and M. H. Levitt, J. Am. Chem. Soc., 2000, 122, 1102-1115.

78. S. Ravindranathan, T. Karlsson, K. Lycknert, G. Widmalm, and M. H. Levitt, J. Magn. Reson., 2001, 151, 136-141.

79. X. Feng, M. Edén, A. Brinkmann, H. Luthman, L. Eriksson, A. Gräslund, O. N. Antzutkin, and M. H. Levitt, J. Am. Chem. Soc., 1997, 119, 12006-12007.

80. P. R. Costa, J. D. Gross, M. Hong, and R. G. Griffin, Chem. Phys. Lett., 1997, 280, 95-103.

81. M. Edén, A. Brinkmann, H. Luthman, L. Eriksson, and M. H. Levitt, J. Magn. Reson., 2000, 144, 266-279. 
82. A. Brinkmann, Ph.D. thesis, Stockholm University, 2001.

83. T. Karlsson, A. Brinkmann, P. J. E. Verdegem, J. Lugtenburg, and M. H. Levitt, Solid State Nucl. Magn. Reson., 1999, 14, 43-58.

84. M. Hong, J. Magn. Reson., 1999, 136, 86-91.

85. M. Carravetta, M. Edén, O. G. Johannessen, H. Luthman, P. J. E. Verdegem, J. Lugtenburg, A. Sebald, and M. H. Levitt, J. Am. Chem. Soc., 2001, 123, $10628-10638$.

86. M. Goldman, V. Fleury, and M. Guron, J. Magn. Reson. A, 1996, 118, 11.

87. P. Tekely and M. Goldman, J. Magn. Reson., 2001, 148, 135-141.

88. M. Mehring and J. S. Waugh, Rev. Sci. Instrum., 1972, 43, 649.

89. M. Carravetta, M. H. Levitt, and A. Brinkmann, to be published.

90. K. Schmidt-Rohr, J. Am. Chem. Soc., 1996, 118, 7601.

91. D. M. Gregory, M. A. Mehta, J. C. Shiels, and G. P. Drobny, J. Chem. Phys., 1997, 107, 28.

92. M. Edén and M. H. Levitt, Chem. Phys. Lett., 1998, 293, 173.

93. H. Geen, R. Graf, A. S. D. Heinrichs, B. S. Hickman, I. Schnell, H. W. Spiess, and J. J. Titman, J. Magn. Reson., 1999, 138, 167.

94. O. N. Antzutkin and R. Tycko, J. Chem. Phys., 1999, 110, 2749.

95. R. Witter, P. Hartmann, J. Vogel, and C. Jager, Solid State Nucl. Magn. Reson., 1998, 13, 189-200.

96. F. Fayon, C. Bessada, J. P. Coutures, and D. Massiot, Inorg. Chem., 1999, 38, 5212-5218.

97. A. Le Sauze, L. Montagne, G. Palavit, F. Fayon, and R. Marchand, J. Non-Cryst. Solids, 2000, 263, 139-145.

98. D. Fayon, D. Massiot, K. Suzuya, and D. L. Price, J. Non-Cryst. Solids, 2001, 283, 88-94.

99. D. F. Schantz, J. Schmedt auf der Günne, H. Koller, and R. F. Lobo, J. Am. Chem. Soc., 2000, 122, 6659-6663.

100. W. A. Dollase, M. Feike, H. Förster, T. Schaller, I. Schnell, A. Sebald, and S. Steuernagel, J. Am. Chem. Soc., 1997, 119, 3807.

101. X. Helluy, C. Marichal, and A. Sebald, J. Phys. Chem. B, 2000, 104, 2836-2845.

102. W. Sommer, J. Gottwald, D. E. Demco, and H. W. Spiess, J. Magn. Reson. A, 1995, 113, 131-134.

103. A. N. Appleyard, R. B. Herbert, P. J. F. Henderson, A. Watts, and P. J. R. Spooner, Biochim. Biophys. Acta, 2000, 1509, 55-64.

104. D. A. Middleton, C. S. Le Du., X. Peng, D. G. Reid, and D. Saunders, J. Am. Chem. Soc., 2000, 122, 1161-1170.

105. C. Glaubitz, M. Carravetta, M. Edén, and M. H. Levitt, in 'Perspectives on Solid State NMR in Biology', eds S. Kühne and H. J. M. de Groot, Kluwer: Dordrecht, 2001.
106. M. Feike, D. E. Demco, R. Graf, J. Gottwald, S. Hafner, and H. W. Spiess, J. Magn. Reson. A, 1996, 122, 214.

107. M. Feike, R. Graf, I. Schnell, C. Jäger, and H. W. Spiess, J. Am. Chem. Soc., 1996, 118, 9631.

108. R. Witter, P. Hartmann, J. Vogel, and C. Jäger, Solid State Nucl. Magn. Reson., 1998, 13, 189.

109. M. G. Munowitz and A. Pines, Science, 1986, 233, 501.

110. D. Suter, S. B. Liu, J. Baum, and A. Pines, Chem. Phys., 1987, 114, 103-109.

111. P. K. Madhu, X. Zhao, and M. H. Levitt, Chem. Phys. Lett., 2001, 346, 142-148.

112. X. Zhao, M. Edén, and M. H. Levitt, Chem. Phys. Lett., 2001, 342, 353-361.

113. X. Zhao, J. L. Sudmeier, W. W. Bachovchin, and M. H. Levitt, J. Am. Chem. Soc., in press.

114. J. D. Gross, P. R. Costa, and R. G. Griffin, J. Chem. Phys., 1998, 108, 7286-7293.

115. M. Hohwy, C. P. Jaroniec, B. Reif, C. M. Rienstra, and R. G. Griffin, J. Am. Chem. Soc., 2000, 122, 3218-3219.

116. B. Reif, M. Hohwy, C. P. Jaroniec, C. M. Rienstra, and R. G. Griffin, J. Magn. Reson., 2000, 145, 132-141.

\section{Acknowledgements}

I would like to thank my research group in Stockholm for helping develop and test these concepts, and for providing experimental results for this review, especially: Young Lee, Oleg Antzutkin, Mattias Edén, Andreas Brinkmann, Marina Carravetta, Xin Zhao, and P. K. Madhu. I would also like to thank Beat Meier, Jerry Chan, Hans Spiess and Yoshitaka Ishii for supplying results prior to publication.

\section{Biographical Sketch}

Malcolm Harris Levitt, $b$ 1957. D. Phil. 1981, University of Oxford, England. Research associate, Weizmann Institute, Israel, 1981-1982, with S. Vega; ETH-Zürich, Switzerland, 1982-1986, with R. R. Ernst; Princeton University, USA, 1986, with W. S. Warren. Research Scientist at the Francis Bitter National Magnet Laboratory, Cambridge, MA, USA 1986-1990. Royal Society Research Fellow at the Interdisciplinary Research Centre for Superconductivity, Cambridge, England, 1990-1991. Lecturer at the Physical Chemistry Division, University of Stockholm, Sweden, 1992-1997. Professor in NMR Methodology, Physical Chemistry Division, University of Stockholm, Sweden, 1997-2001. Professor in Physical Chemistry, Southampton University, England, from 2001. Approx 120 publications. Current research interests: methodology and theory of NMR and its applications, especially to biological materials. 